\title{
Stereoselective Construction of the Tricyclic Core of Neoliacinic Acid
}

\author{
J. Stephen Clark, ${ }^{\dagger *}$ Carl A. Baxter, ${ }^{\ddagger}$ Alexander G. Dossetter, ${ }^{\ddagger}$ \\ Stéphane Poigny, ${ }^{\ddagger}$ José L. Castro ${ }^{\#}$ and William G. Whittingham ${ }^{\S}$
}

${ }^{\dagger}$ WestCHEM, Department of Chemistry, Joseph Black Building, University of Glasgow, University Avenue, Glasgow, G12 8QQ, United Kingdom

${ }^{\sharp}$ School of Chemistry, University of Nottingham, University Park, Nottingham, NG7 2RD, UK

\# Merck Sharp \& Dohme, Neuroscience Research Centre, Terlings Park, Harlow, Essex, CM20 2QR, United Kingdom. Current address: GlaxoSmithKline, Neurology CEDD, New Frontiers Science Park, Third Avenue, Harlow, Essex CM19 5AW, United Kingdom

$\S$ Crop Protection Research Chemistry, Syngenta, Jealott's Hill International Research Centre, Bracknell, Berkshire, RG42 6EY, United Kingdom

email: stephenc@chem.gla.ac.uk

\section{Supporting Information}




\section{Contents}

$\begin{array}{ll}\text { General Information } & \text { S04 }\end{array}$

$\begin{array}{ll}{ }^{1} \mathrm{H} \text { NMR spectrum of compound } 7 & \text { S05 }\end{array}$

${ }^{13} \mathrm{C}$ NMR spectrum of compound $7 \quad$ S06

$\begin{array}{ll}{ }^{1} \mathrm{H} \text { NMR spectrum of compound } \mathbf{8} & \text { S07 }\end{array}$

$\begin{array}{ll}{ }^{13} \mathrm{C} \text { NMR spectrum of compound } \mathbf{8} & \text { S08 }\end{array}$

$\begin{array}{ll}{ }^{1} \mathrm{H} \text { NMR spectrum of compound } \mathbf{1 1} & \text { S09 }\end{array}$

$\begin{array}{lr}{ }^{13} \mathrm{C} \text { NMR spectrum of compound } \mathbf{1 1} & \text { S10 }\end{array}$

$\begin{array}{ll}{ }^{1} \mathrm{H} \text { NMR spectrum of compound } \mathbf{1 2} & \mathrm{S} 11\end{array}$

${ }^{13} \mathrm{C}$ NMR spectrum of compound $\mathbf{1 2} \quad \mathrm{S} 12$

$\begin{array}{ll}{ }^{1} \mathrm{H} \text { NMR spectrum of compound } \mathbf{1 3} & \mathrm{S} 13\end{array}$

$\begin{array}{ll}{ }^{13} \mathrm{C} \text { NMR spectrum of compound } \mathbf{1 3} & \mathrm{S} 14\end{array}$

$\begin{array}{ll}{ }^{1} \mathrm{H} \text { NMR spectrum of compound } \mathbf{1 5} & \text { S15 }\end{array}$

${ }^{13} \mathrm{C}$ NMR spectrum of compound $\mathbf{1 5} \quad$ S16

$\begin{array}{ll}{ }^{1} \mathrm{H} \text { NMR spectrum of compound } \mathbf{1 6} & \mathrm{S} 17\end{array}$

$\begin{array}{lr}{ }^{13} \mathrm{C} \text { NMR spectrum of compound } \mathbf{1 6} & \mathrm{S} 18\end{array}$

$\begin{array}{lr}{ }^{1} \mathrm{H} \text { NMR spectrum of compound } \mathbf{1 7} & \text { S19 }\end{array}$

$\begin{array}{ll}{ }^{13} \mathrm{C} \text { NMR spectrum of compound } \mathbf{1 7} & \text { S20 }\end{array}$

$\begin{array}{ll}{ }^{1} \mathrm{H} \text { NMR spectrum of compound } \mathbf{1 8} & \text { S21 }\end{array}$

$\begin{array}{ll}{ }^{13} \mathrm{C} \text { NMR spectrum of compound } \mathbf{1 8} & \text { S22 }\end{array}$

$\begin{array}{ll}{ }^{1} \mathrm{H} \text { NMR spectrum of compound } 19 & \text { S23 }\end{array}$

${ }^{13} \mathrm{C}$ NMR spectrum of compound $19 \quad$ S24

$\begin{array}{ll}{ }^{1} \mathrm{H} \text { NMR spectrum of compound } 21 & \text { S25 }\end{array}$

$\begin{array}{ll}{ }^{13} \mathrm{C} \text { NMR spectrum of compound } \mathbf{2 1} & \text { S26 }\end{array}$

$\begin{array}{ll}{ }^{1} \mathrm{H} \text { NMR spectrum of compound } \mathbf{2 3} & \text { S27 }\end{array}$

$\begin{array}{ll}{ }^{13} \mathrm{C} \text { NMR spectrum of compound } \mathbf{2 3} & \text { S28 }\end{array}$

$\begin{array}{ll}{ }^{1} \mathrm{H} \text { NMR spectrum of compound } \mathbf{2 4} & \text { S29 }\end{array}$

$\begin{array}{lr}{ }^{13} \mathrm{C} \text { NMR spectrum of compound } 24 & \text { S30 }\end{array}$

$\begin{array}{ll}{ }^{1} \mathrm{H} \text { NMR spectrum of compound } \mathbf{2 5} & \text { S31 }\end{array}$

${ }^{13}$ C NMR spectrum of compound $25 \quad$ S32

$\begin{array}{ll}{ }^{1} \mathrm{H} \text { NMR spectrum of compound } \mathbf{2 6} & \text { S33 }\end{array}$

$\begin{array}{ll}{ }^{13} \mathrm{C} \text { NMR spectrum of compound } \mathbf{2 6} & \text { S34 }\end{array}$

$\begin{array}{ll}{ }^{1} \mathrm{H} \text { NMR spectrum of compound } 27 & \text { S35 }\end{array}$

${ }^{13} \mathrm{C}$ NMR spectrum of compound $27 \quad$ S36

$\begin{array}{ll}{ }^{1} \mathrm{H} \text { NMR spectrum of compound } \mathbf{2 8} & \text { S37 }\end{array}$ 


\section{J. S. Clark et al. - Supporting Information}

${ }^{13} \mathrm{C}$ NMR spectrum of compound 28

${ }^{1} \mathrm{H}$ NMR spectrum of compound 29

S39

${ }^{13} \mathrm{C}$ NMR spectrum of compound 29

S40

${ }^{1} \mathrm{H}$ NMR spectrum of compound $\mathbf{3 0}$

S41

${ }^{13} \mathrm{C}$ NMR spectrum of compound $\mathbf{3 0}$ S42

${ }^{1} \mathrm{H}$ NMR spectrum of compound $\mathbf{3 3}$ S43

${ }^{13} \mathrm{C}$ NMR spectrum of compound $\mathbf{3 3}$ S44

${ }^{1} \mathrm{H}$ NMR spectrum of compound 34 S45

${ }^{13} \mathrm{C}$ NMR spectrum of compound 34 S46

${ }^{1} \mathrm{H}$ NMR spectrum of compound $\mathbf{3 5}$ S47

${ }^{13} \mathrm{C}$ NMR spectrum of compound $\mathbf{3 5}$ S48

${ }^{1} \mathrm{H}$ NMR spectrum of compound $37\left(\mathrm{CDCl}_{3}\right)$ S49

${ }^{1} \mathrm{H}$ NMR spectrum of compound $37\left(\mathrm{C}_{6} \mathrm{D}_{6}\right)$ $\mathrm{S} 50$

${ }^{13} \mathrm{C}$ NMR spectrum of compound 37 S51

${ }^{1} \mathrm{H}$ NMR spectrum of compound $\mathbf{3 8}$ S52

${ }^{13} \mathrm{C}$ NMR spectrum of compound $\mathbf{3 8}$ S53

${ }^{1} \mathrm{H}$ NMR spectrum of compound 39 S54

${ }^{13} \mathrm{C}$ NMR spectrum of compound 39 S55

${ }^{1} \mathrm{H}$ NMR spectrum of compound $\mathbf{4 1}$ S56

${ }^{13} \mathrm{C}$ NMR spectrum of compound $\mathbf{4 1}$ S57

${ }^{1} \mathrm{H}$ NMR spectrum of compound $\mathbf{4 3}$ S58

${ }^{13} \mathrm{C}$ NMR spectrum of compound $\mathbf{4 3}$ S59

${ }^{1} \mathrm{H}$ NMR spectrum of compound 46 S60

${ }^{13} \mathrm{C}$ NMR spectrum of compound 46 S61 


\section{J. S. Clark et al. - Supporting Information}

\section{General Information}

All ${ }^{1} \mathrm{H}$ NMR spectra were recorded at ambient temperature and data is expressed in terms of chemical shift (ppm) relative to residual $\mathrm{CHCl}_{3}$ or tetramethylsilane on the $\delta$ scale, integration, multiplicity $(\mathrm{br}=$ broad, $\mathrm{s}=$ singlet, $\mathrm{d}=$ doublet, $\mathrm{t}=$ triplet, $\mathrm{q}=$ quartet, $\mathrm{sx}=$ sextet, $\mathrm{m}=$ multiplet or combinations thereof), coupling constant and assignment. All ${ }^{13} \mathrm{C}$ NMR spectra were recorded on a at ambient temperature and data is expressed in terms of chemical shift (ppm) relative to residual $\mathrm{CHCl}_{3}$ on the $\delta$ scale followed by assignment $\left(\mathrm{C}, \mathrm{CH}, \mathrm{CH}_{2}, \mathrm{CH}_{3}\right)$. The ${ }^{13} \mathrm{C}$ NMR assignments were made on the basis of data obtained from DEPT 135 and DEPT 90 pulse experiments and HMQC experiments. IR spectra were recorded as liquid film samples ( $\mathrm{NaCl}$ plates) or as solutions (solution cell) in chloroform in the range $4000-600 \mathrm{~cm}^{-1}$ at ambient temperature. High resolution mass spectra were obtained using EI, CI, FAB or ES techniques.

Solvents were distilled prior to use employing standard methods: THF by distillation from potassium-benzophenone, diethyl ether by distillation from sodium-benzophenone, $\mathrm{CH}_{2} \mathrm{Cl}_{2}$ by distillation from $\mathrm{CaH}_{2}$. Triethylamine and 2,6-lutidine were distilled from $\mathrm{KOH}$ under nitrogen and were stored under nitrogen over $\mathrm{KOH}$. All reagents were used as supplied unless otherwise stated.

Thin layer chromatography was performed using Kiesegel $60 \mathrm{~F}_{254}$ plates. Visualisation was by UV light and staining with ethanolic anisaldehyde or ceric ammonium molybdate with heat. Flash column chromatography was performed using silica gel 35-70 $\mu \mathrm{m}, 60 \mathrm{~A}$.

Magnesium for all Grignard reactions was flame dried under an atmosphere of nitrogen or argon in a flask equipped with an olive-style magnetic stirrer bar then dry stirred for $2 \mathrm{~h}$.

All reactions were performed under an atmosphere of nitrogen in oven-dried glassware. Reagents were purchased and used without further purification unless indicated otherwise. The complex $\mathrm{Cu}(\mathrm{hfacac})_{2}$ was purchased and used as supplied. The complex $\mathrm{Rh}_{2}\left(\mathrm{HNCOCF}_{3}\right)_{4}$ was prepared according to the procedure of Dennis et al. ${ }^{1}$

\section{References}

1. Dennis, A. M.; Korp, J. D.; Bernal, I.; Howard, R. A.; Bear, J. L. Inorg. Chem. 1983, 22, 1522. 
J. S. Clark et al. - Supporting Information
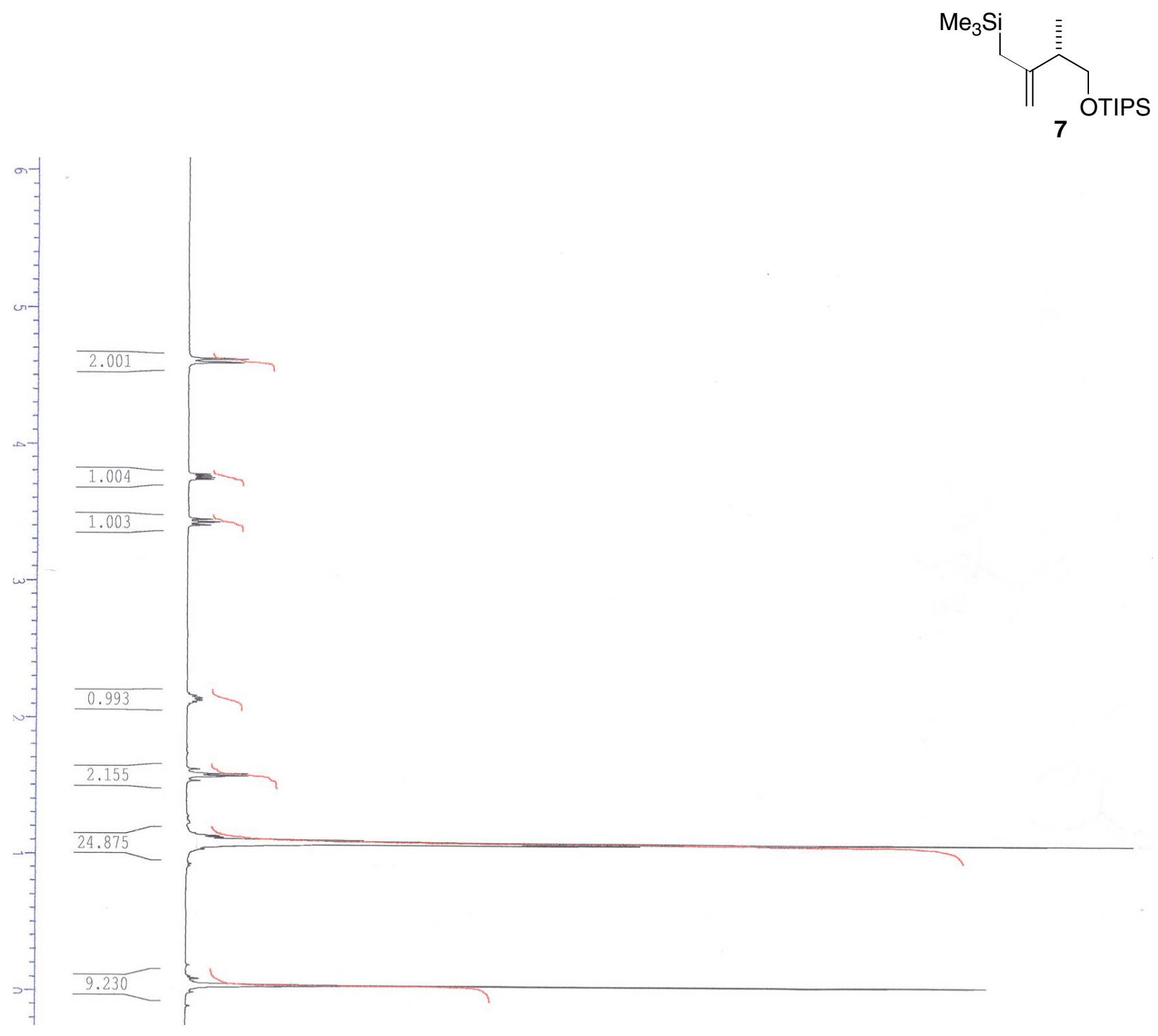
J. S. Clark et al. - Supporting Information
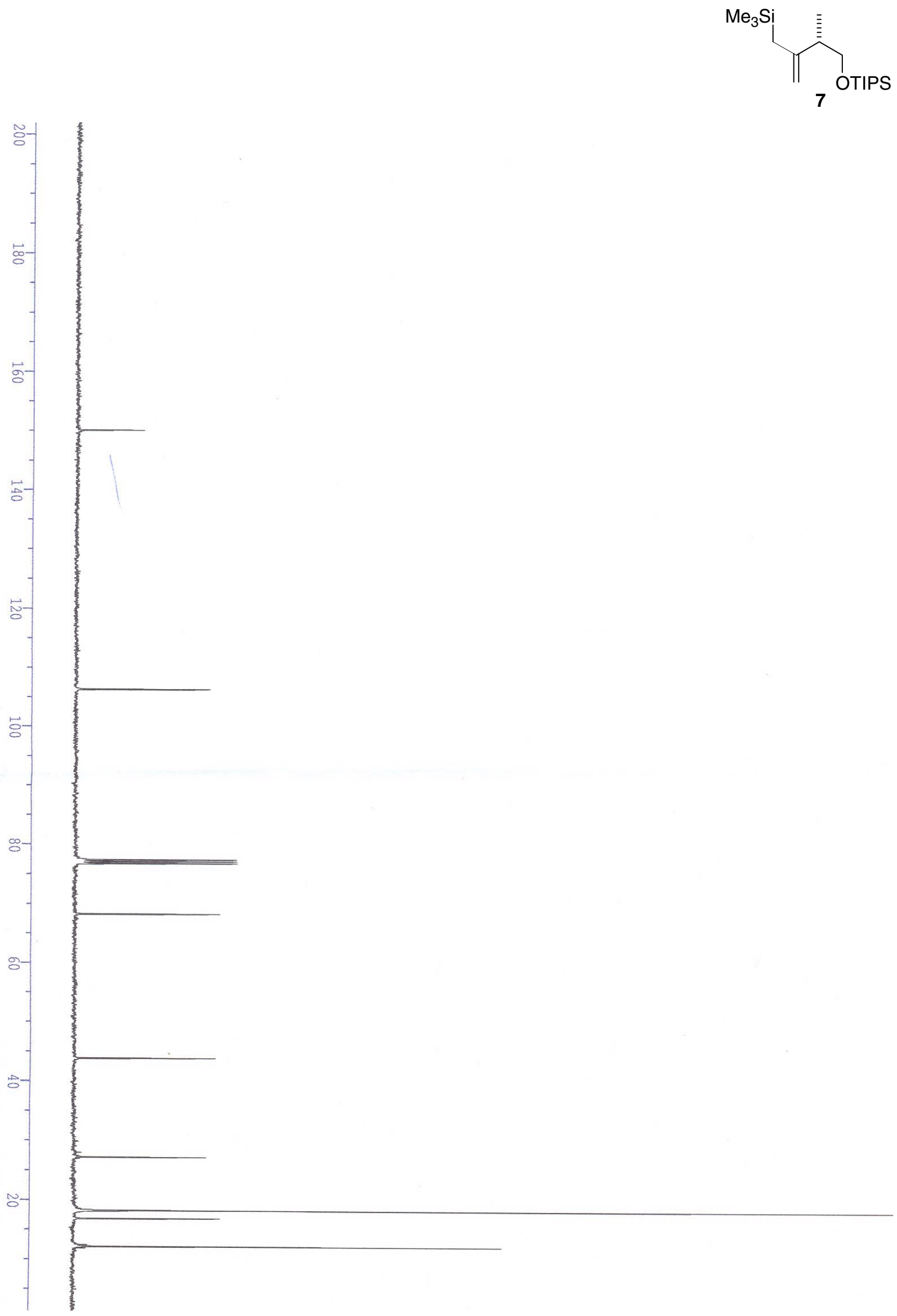
J. S. Clark et al. - Supporting Information
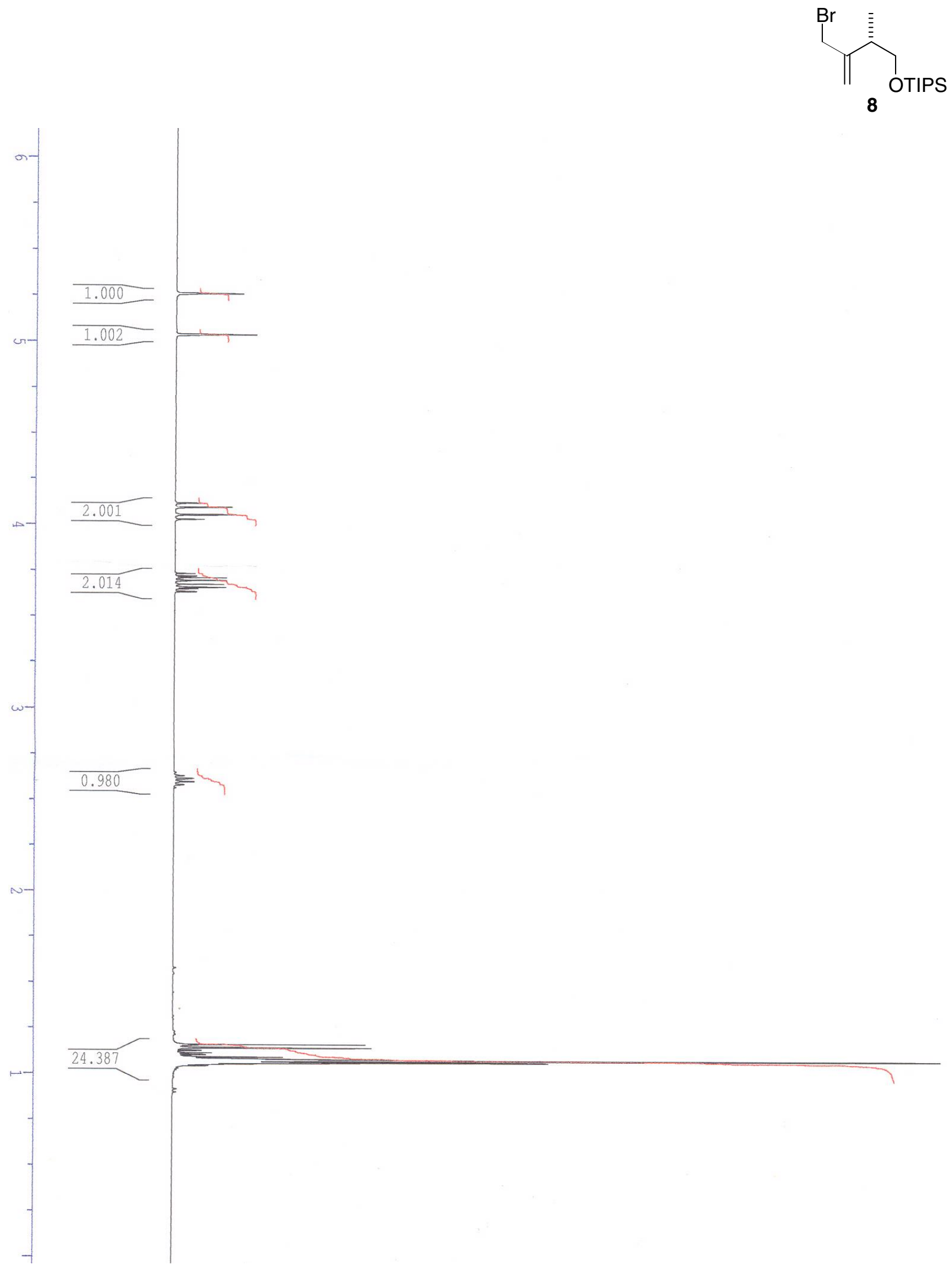
J. S. Clark et al. - Supporting Information
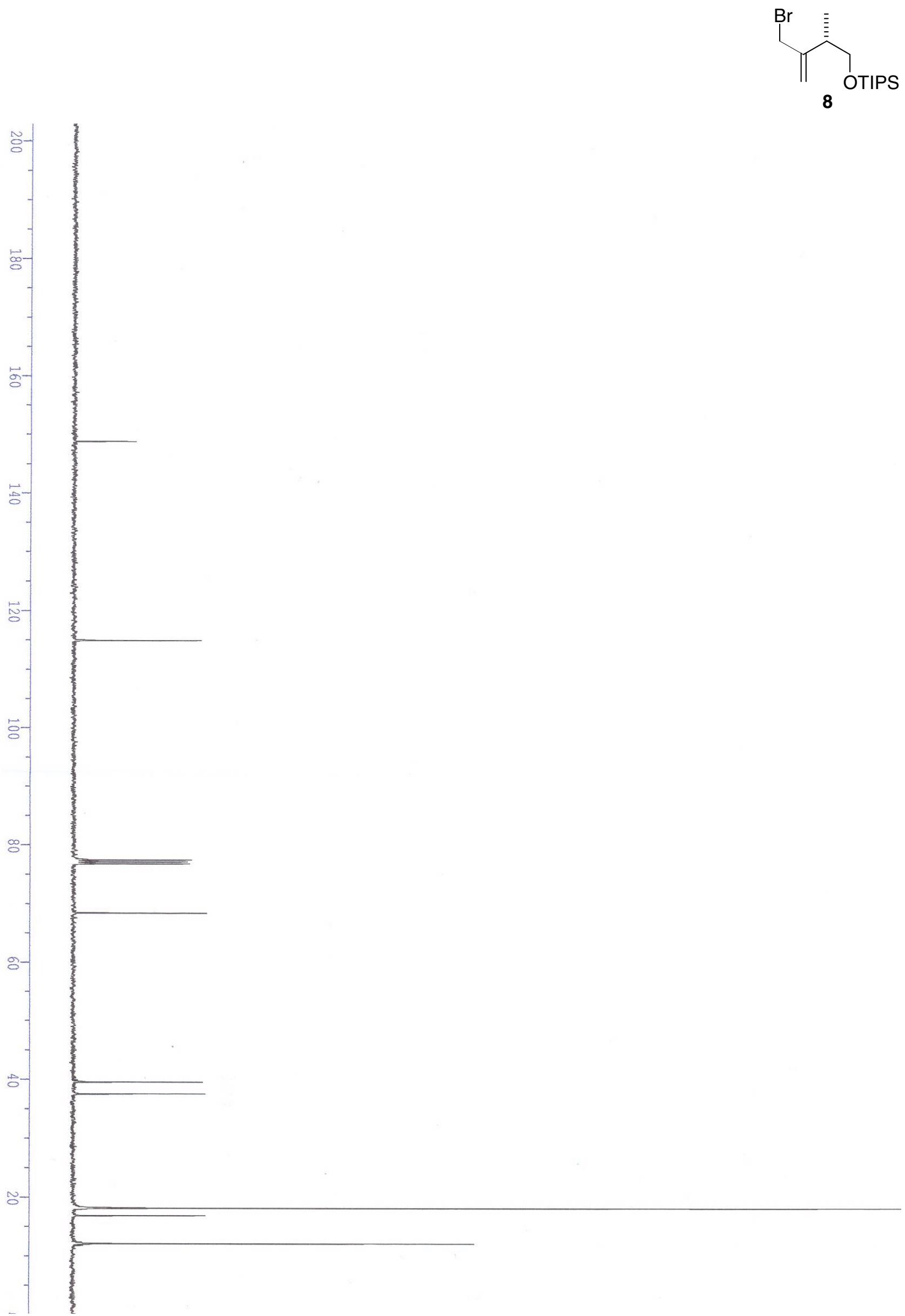


\section{J. S. Clark et al. - Supporting Information}

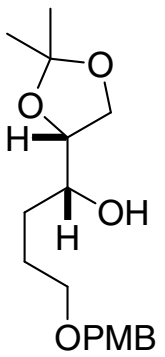

11

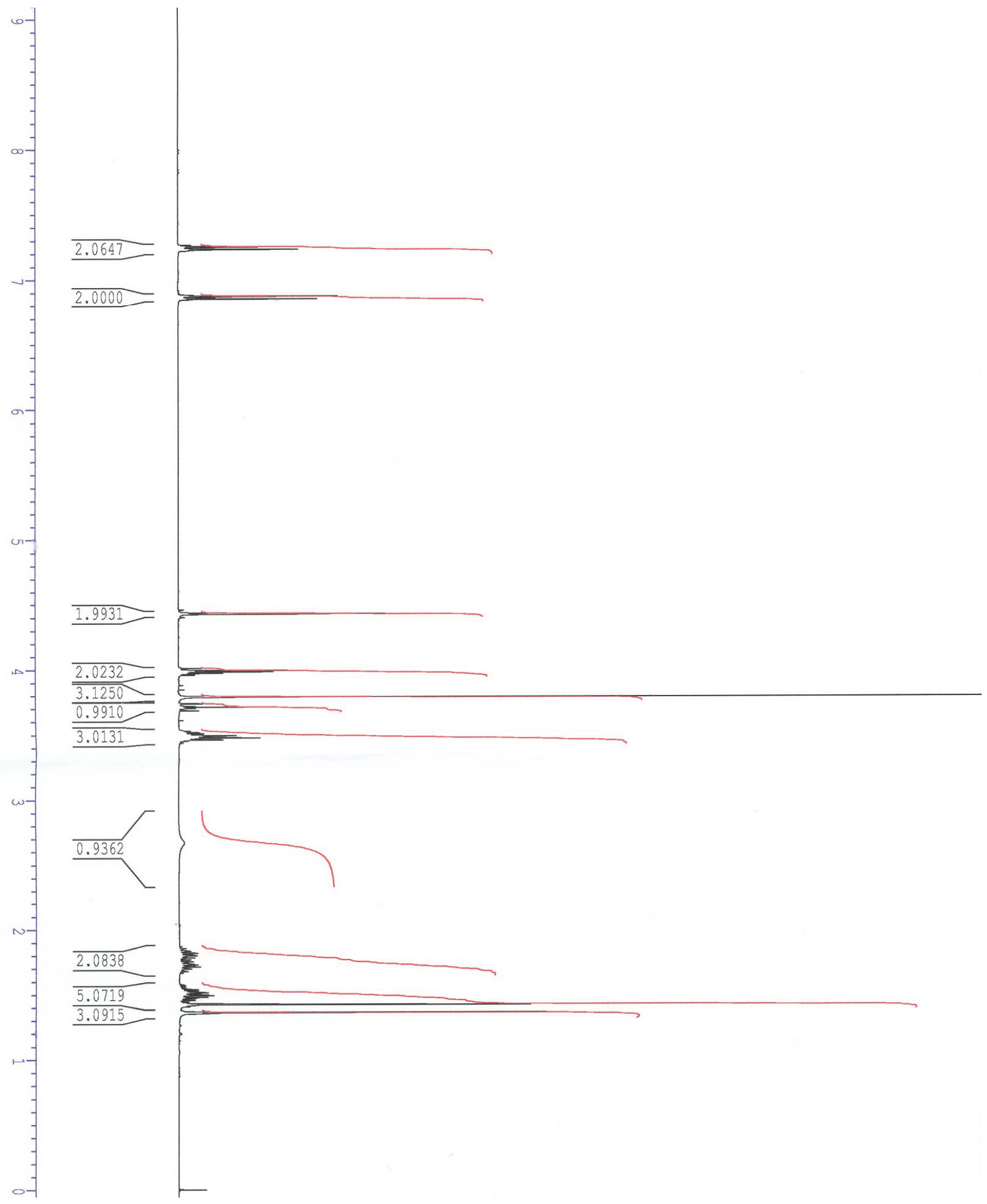


J. S. Clark et al. - Supporting Information<smiles>CCCCCC(O)C1COC(C)(C)O1</smiles>

11

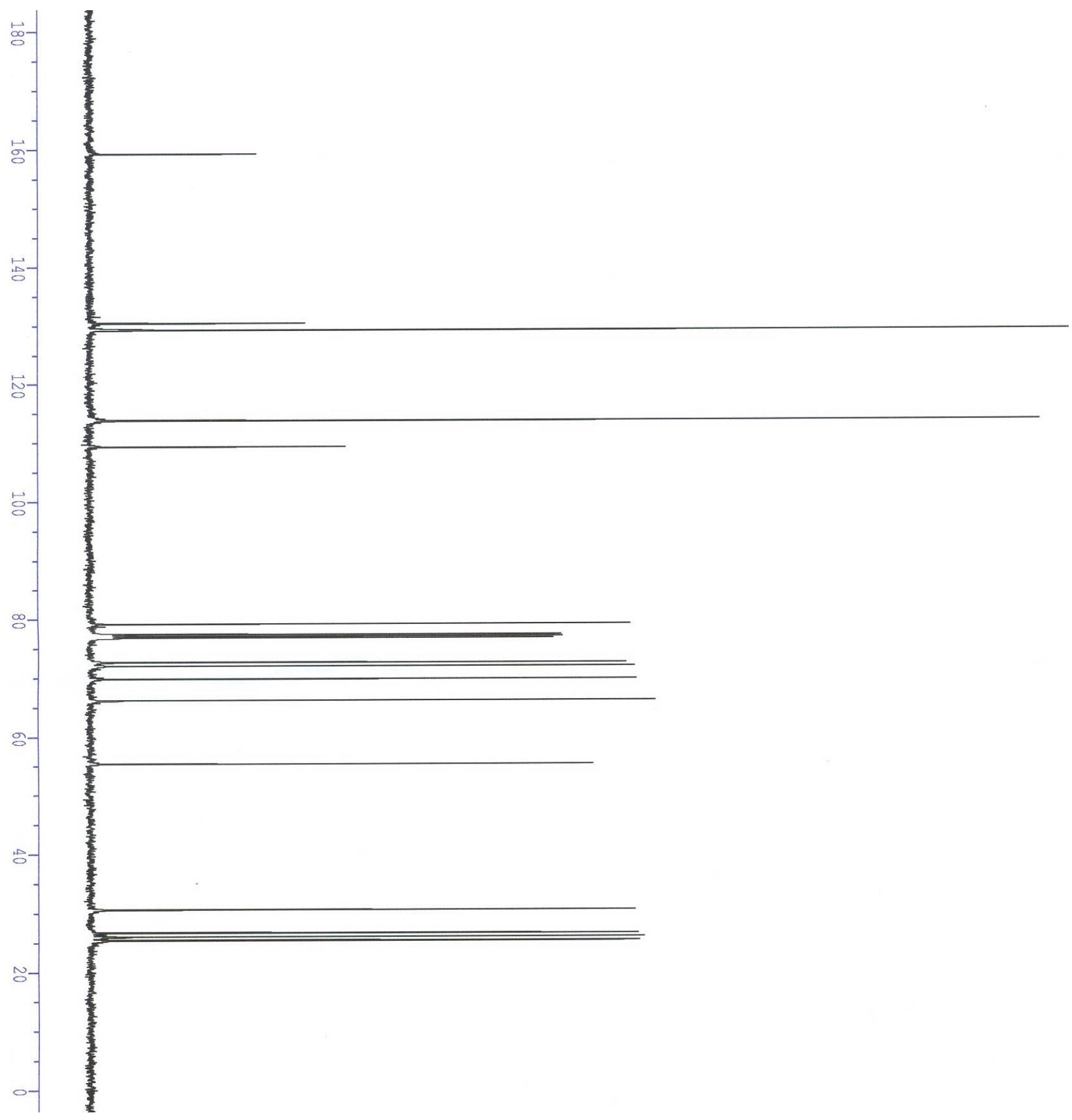

S10 
J. S. Clark et al. - Supporting Information

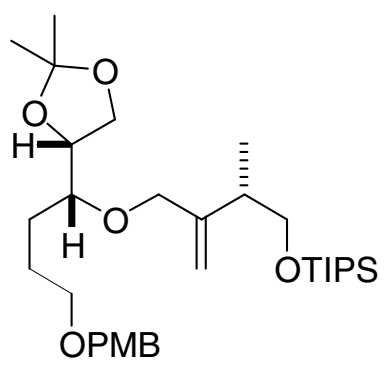

12

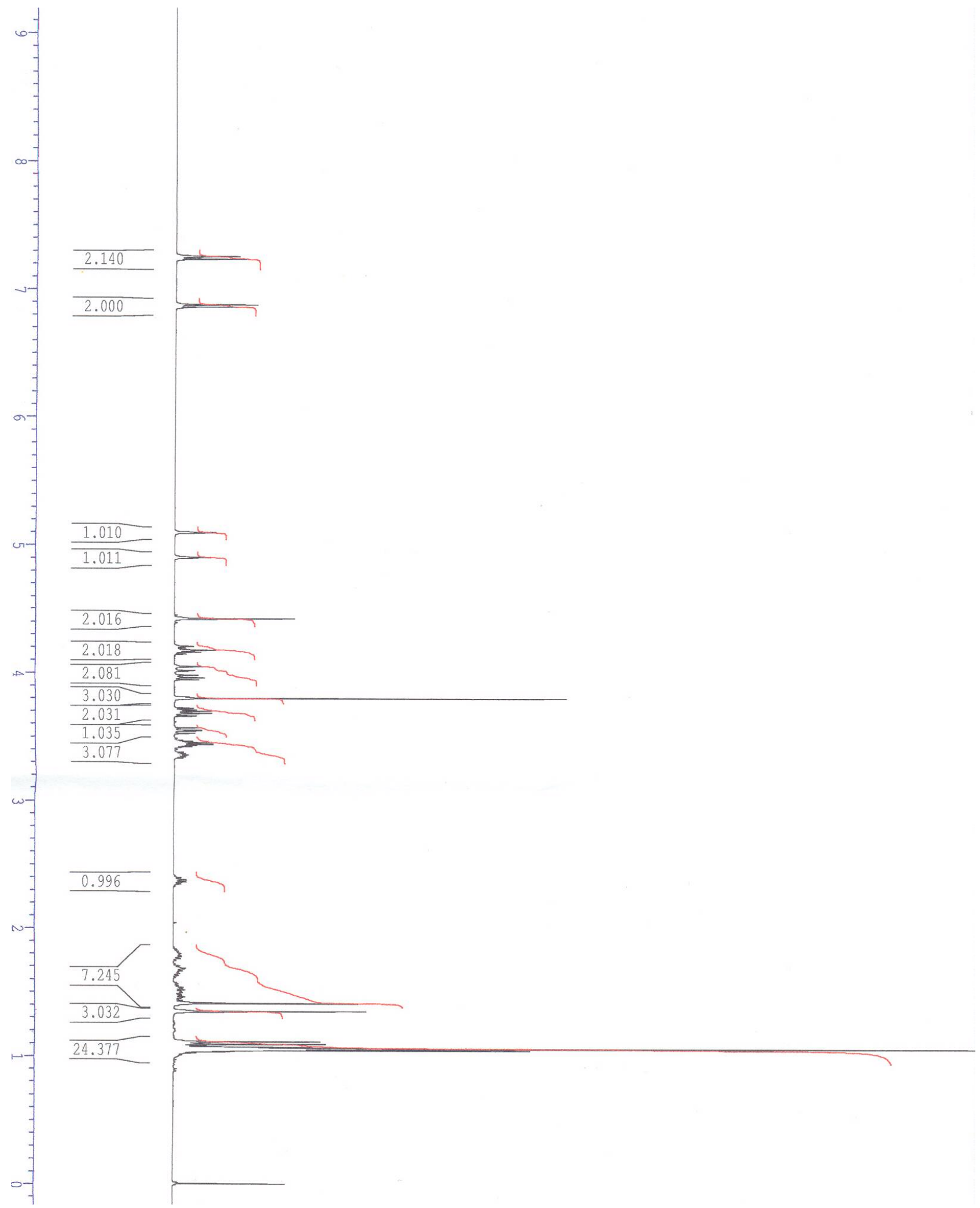




\section{J. S. Clark et al. - Supporting Information}

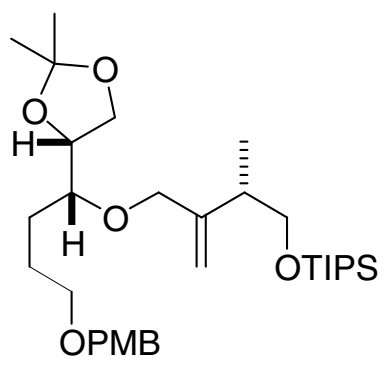

12

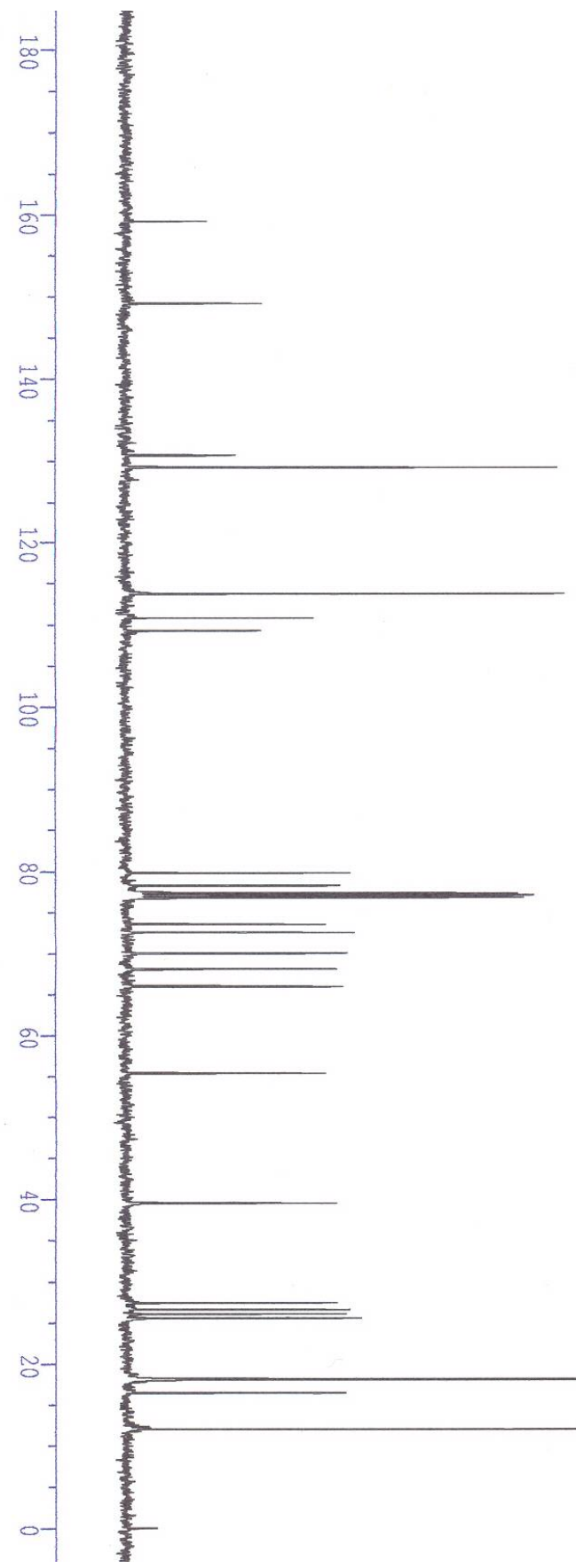


J. S. Clark et al. - Supporting Information

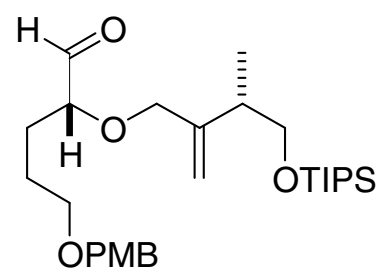

13

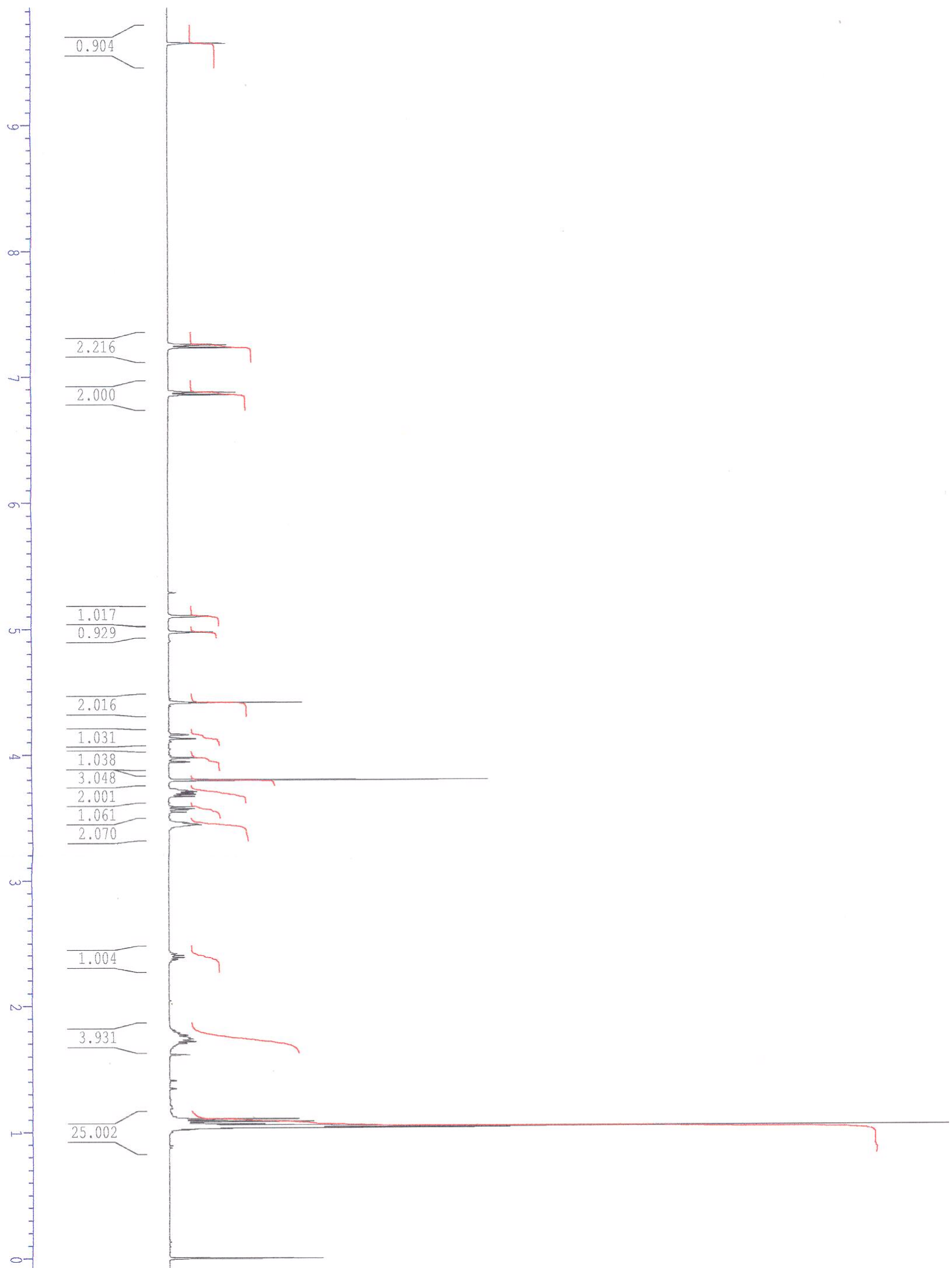




\section{J. S. Clark et al. - Supporting Information}

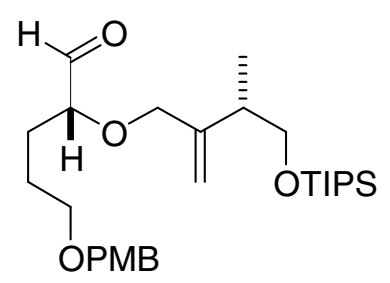

13

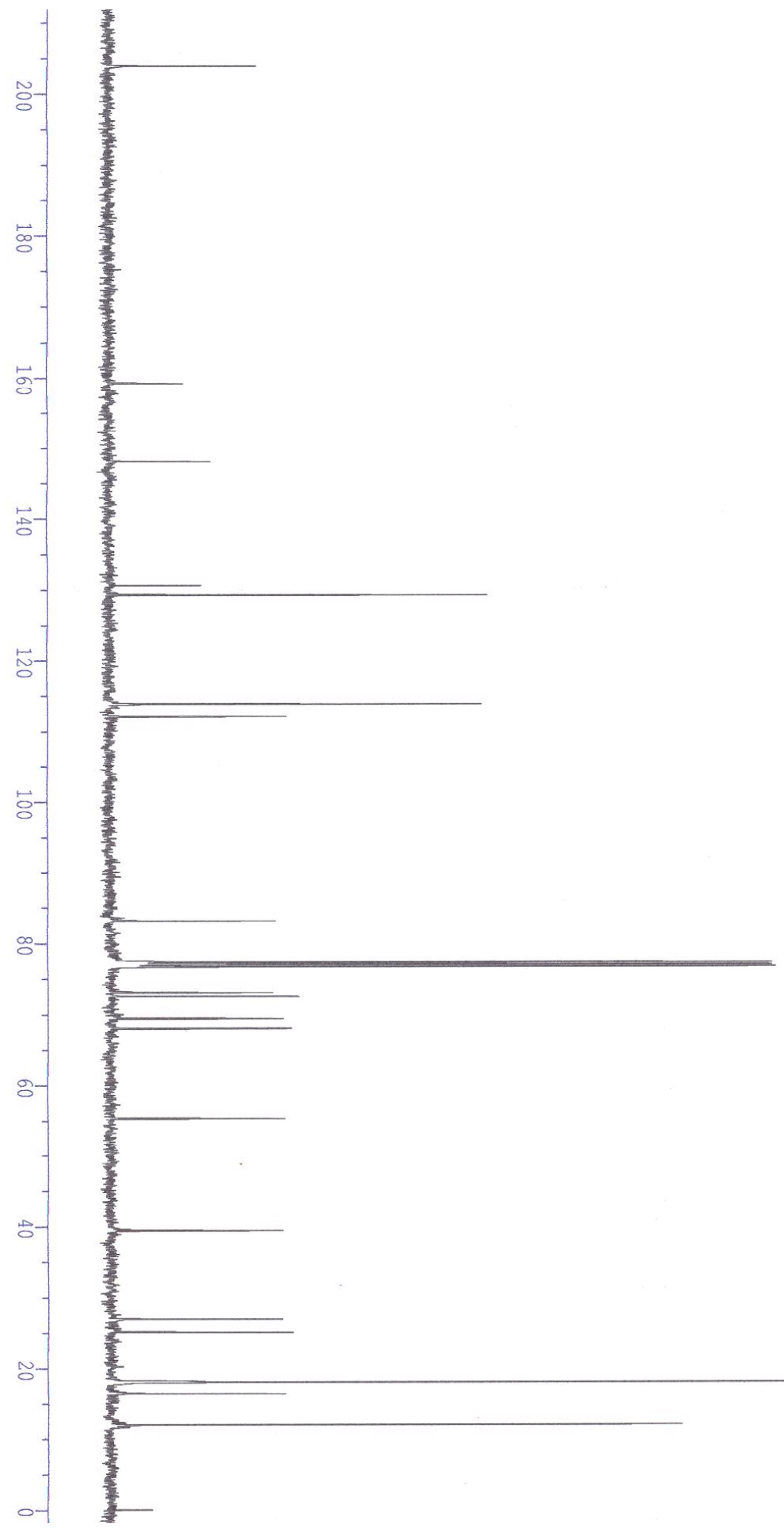




\section{J. S. Clark et al. - Supporting Information}

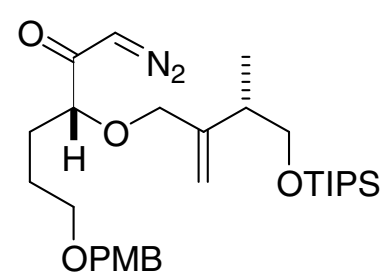

15

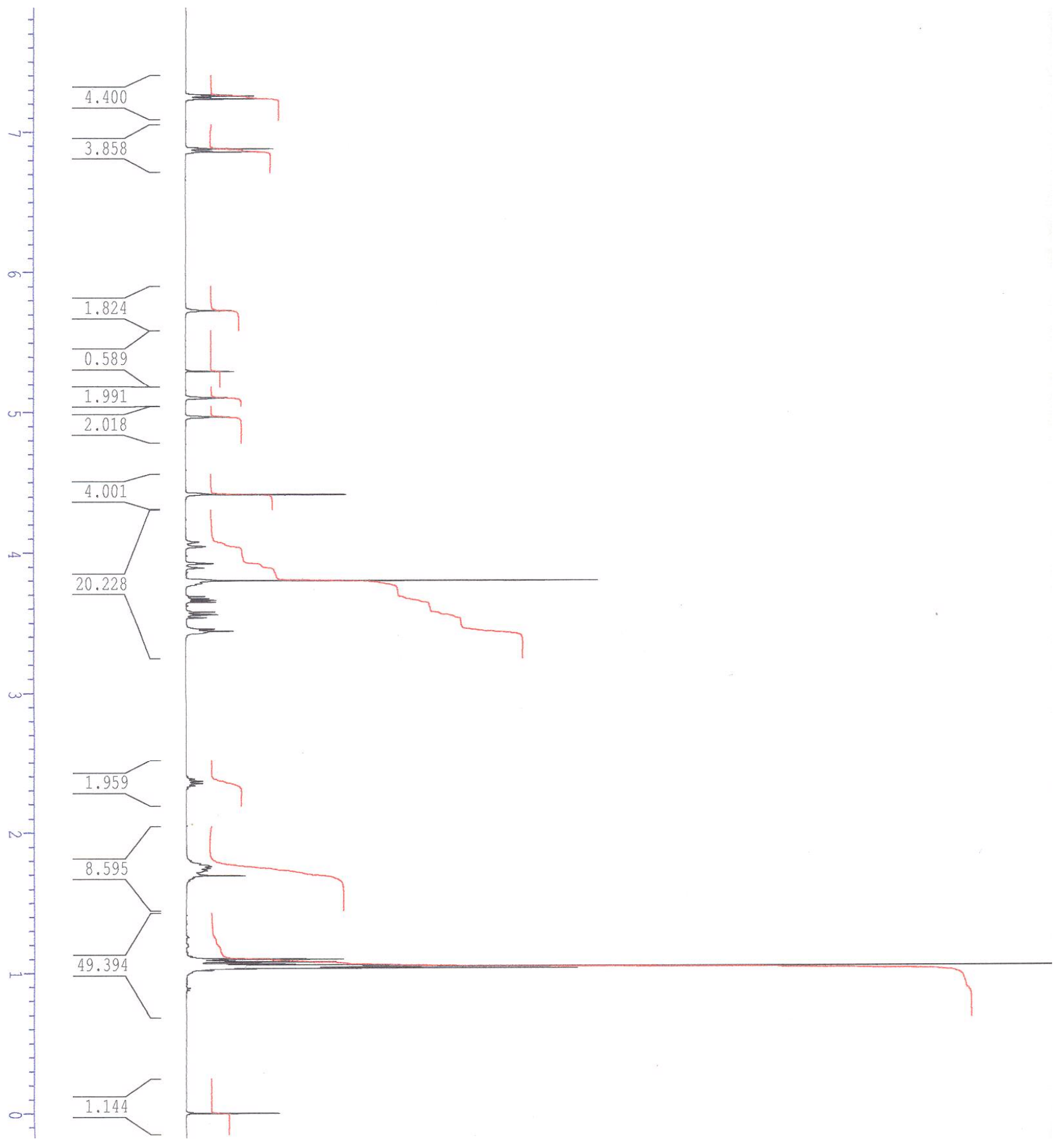


J. S. Clark et al. - Supporting Information
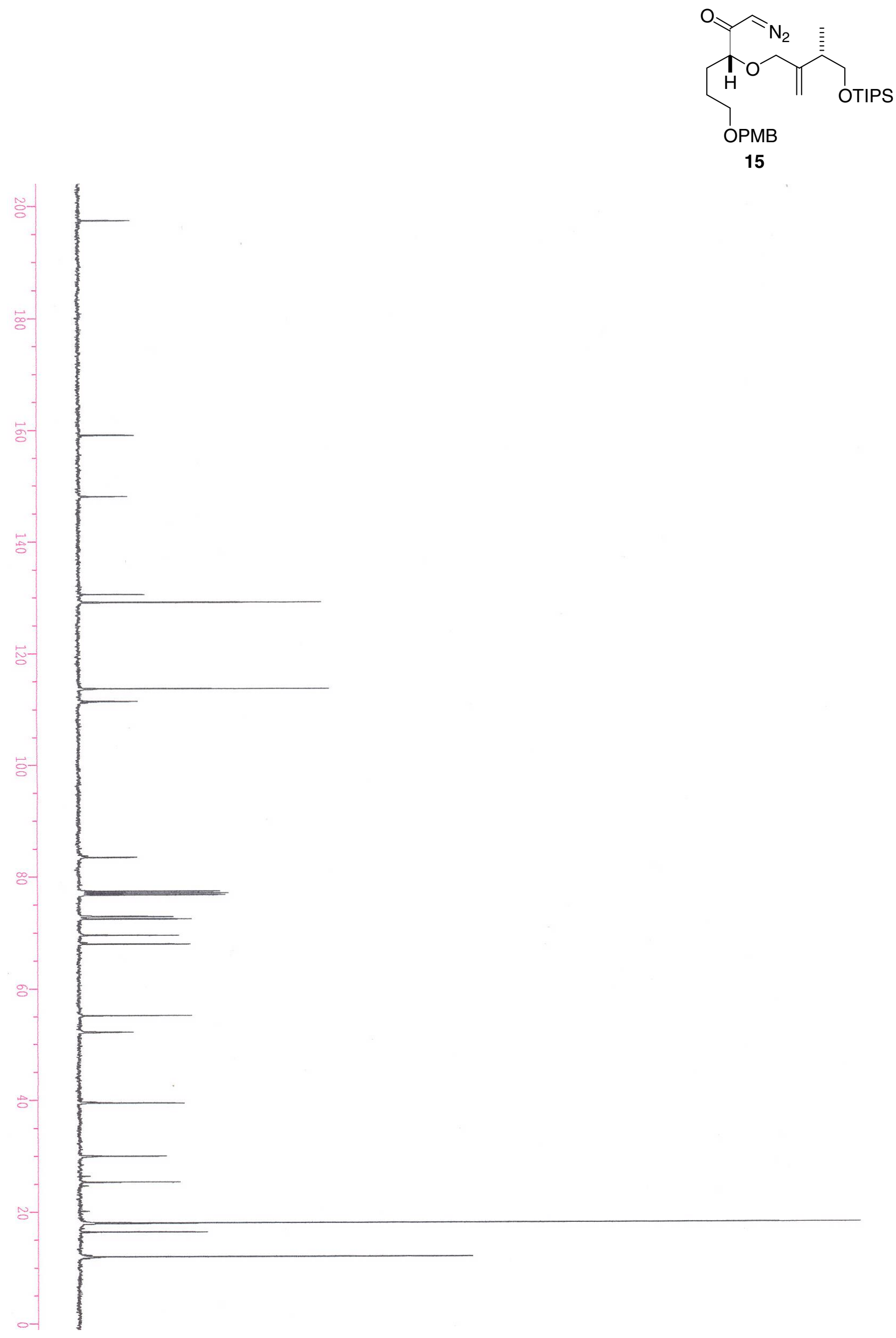

S16 


\section{J. S. Clark et al. - Supporting Information}
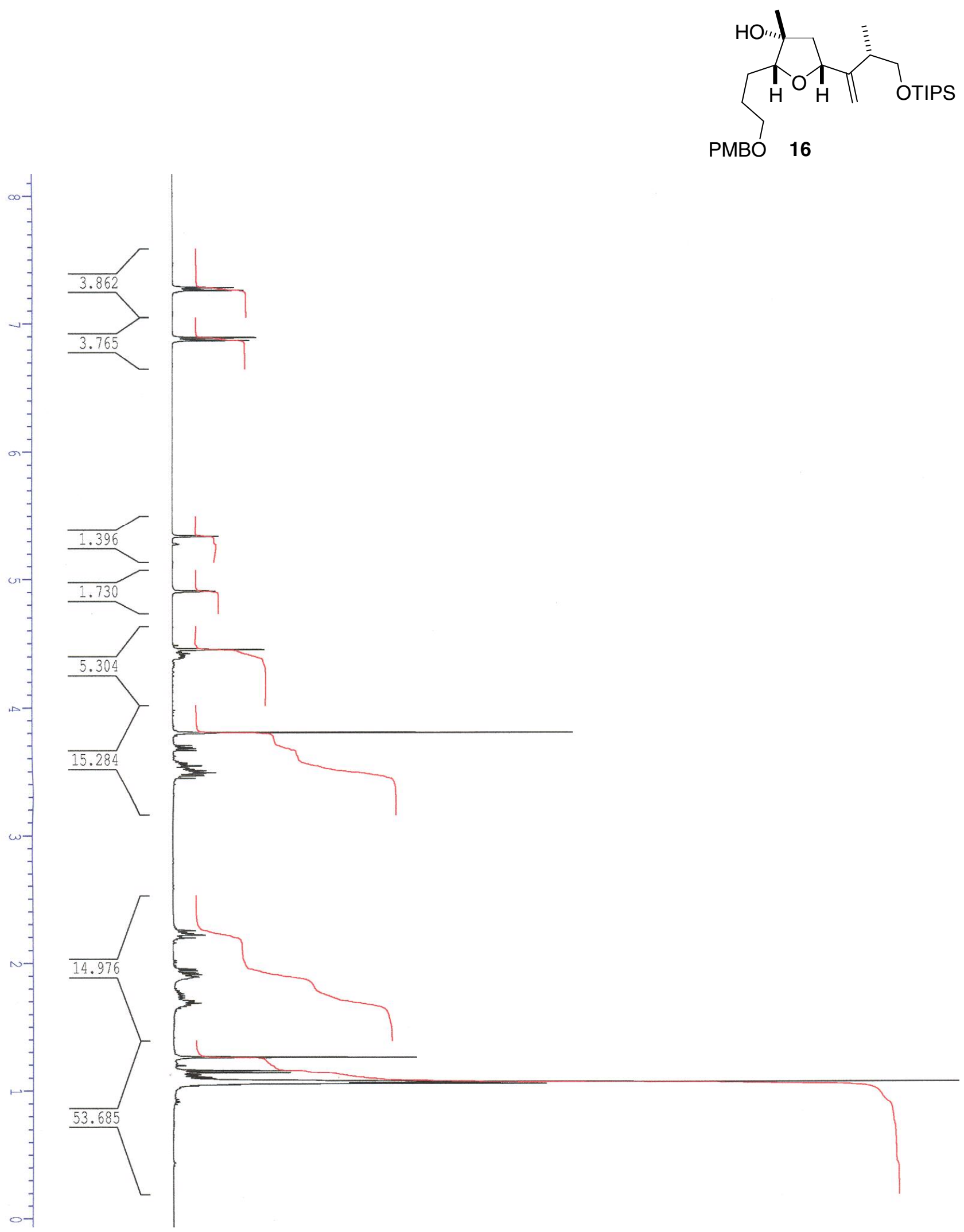
J. S. Clark et al. - Supporting Information
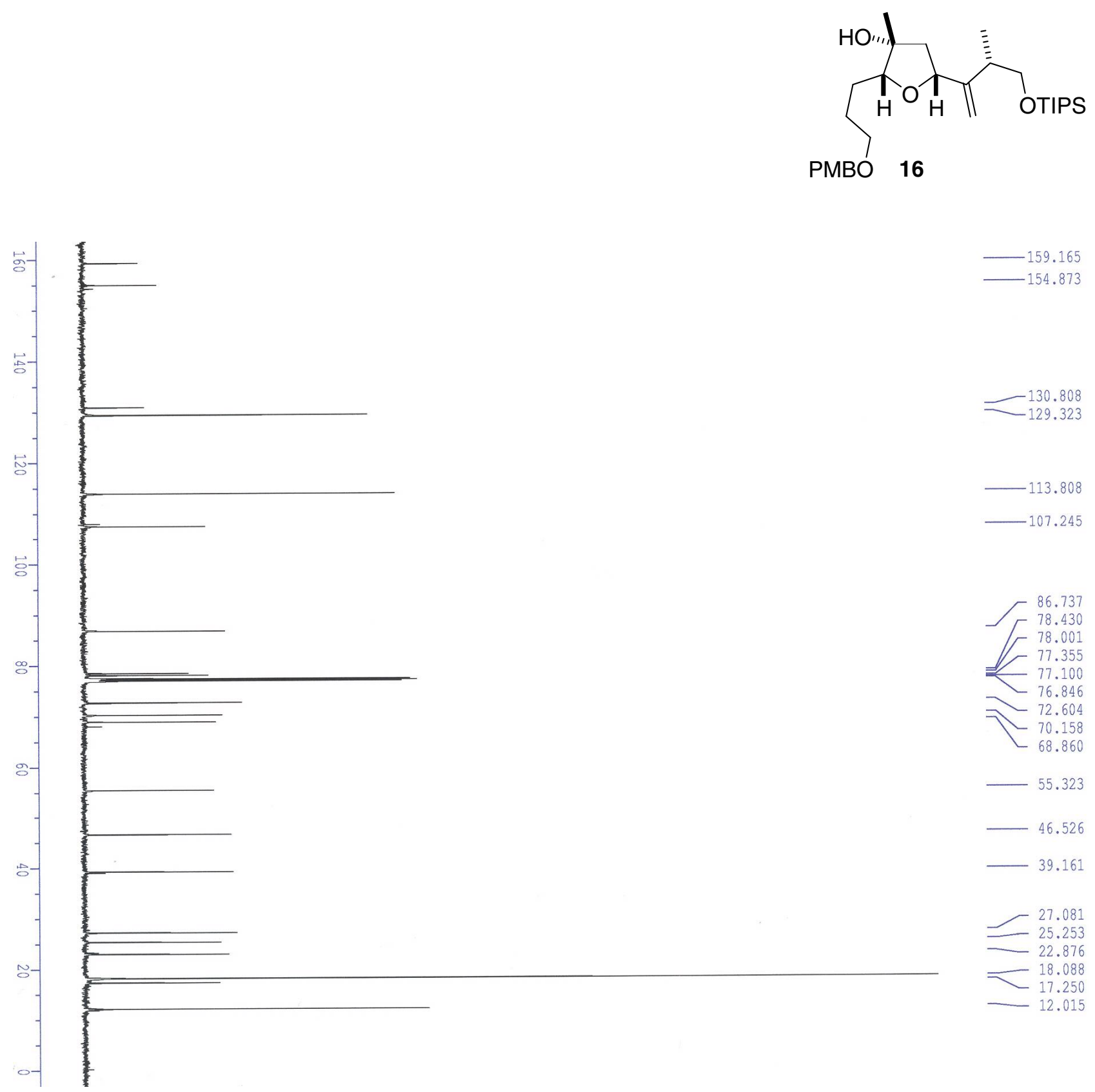

$=130.808$

$-113.808$

$-107.245$

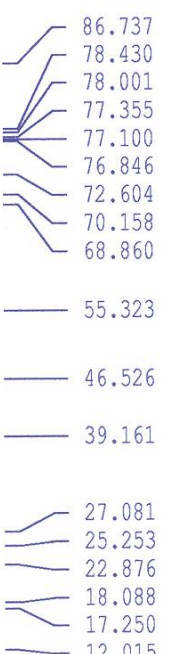




\section{J. S. Clark et al. - Supporting Information}
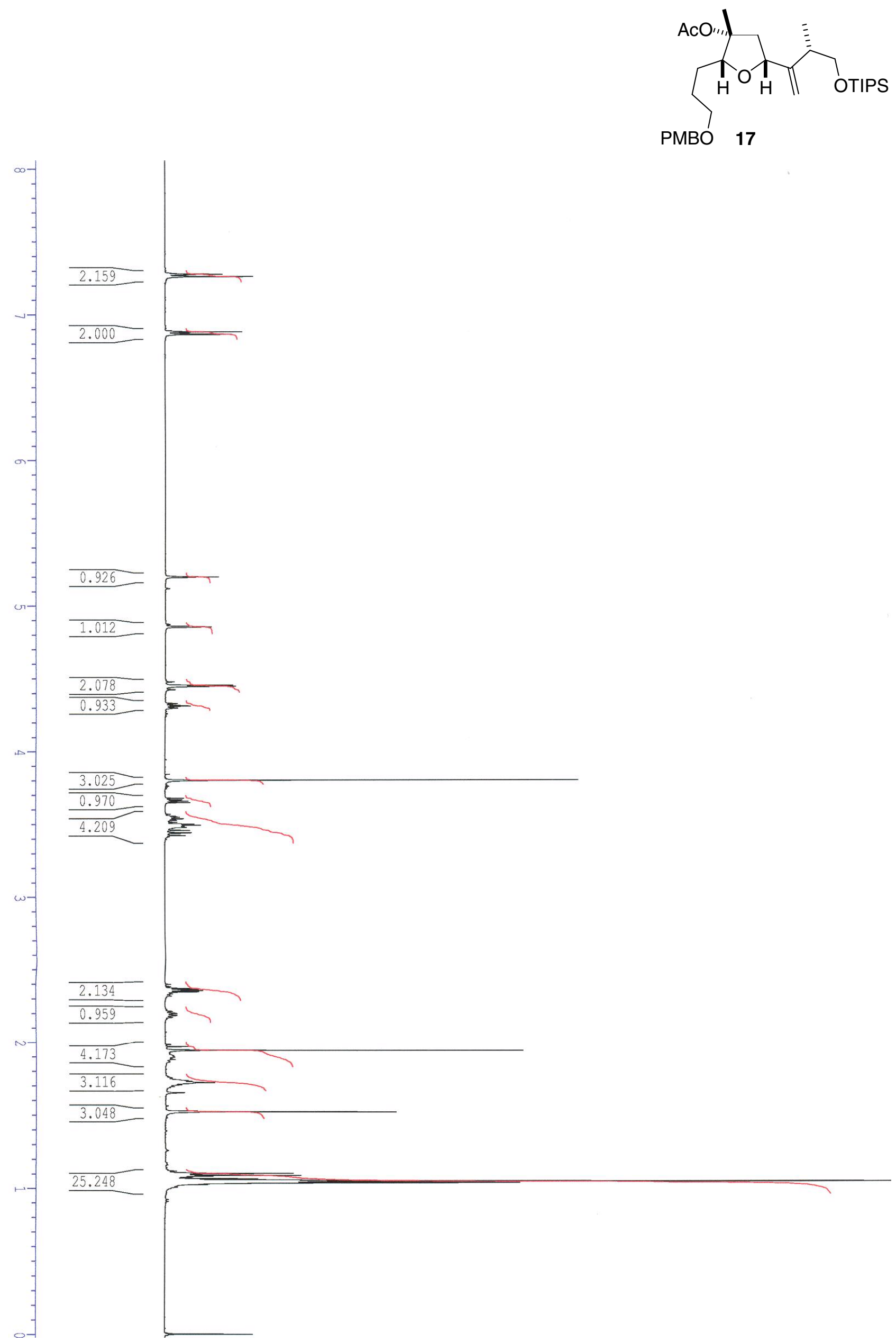


\section{J. S. Clark et al. - Supporting Information}

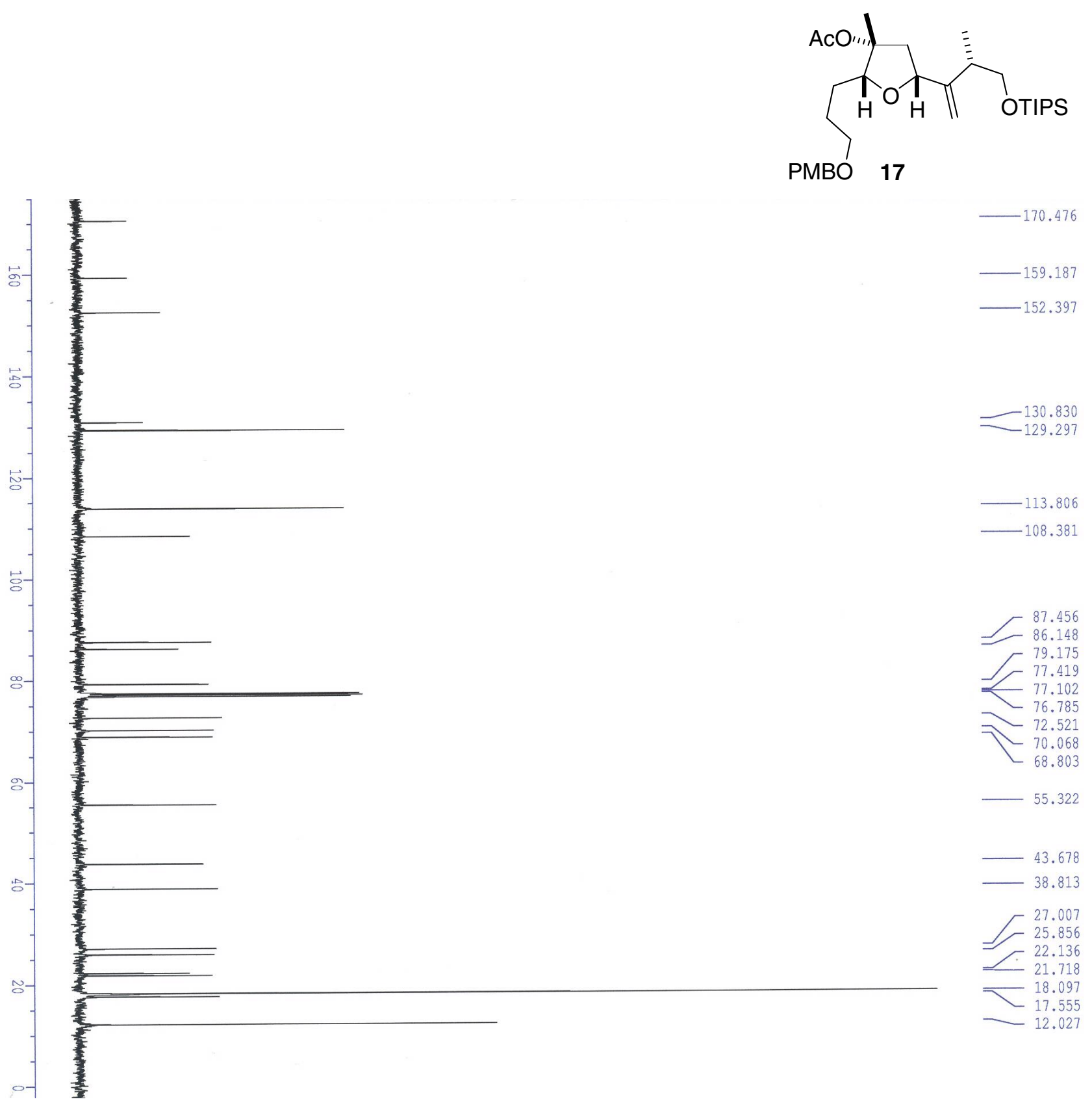




\section{J. S. Clark et al. - Supporting Information}
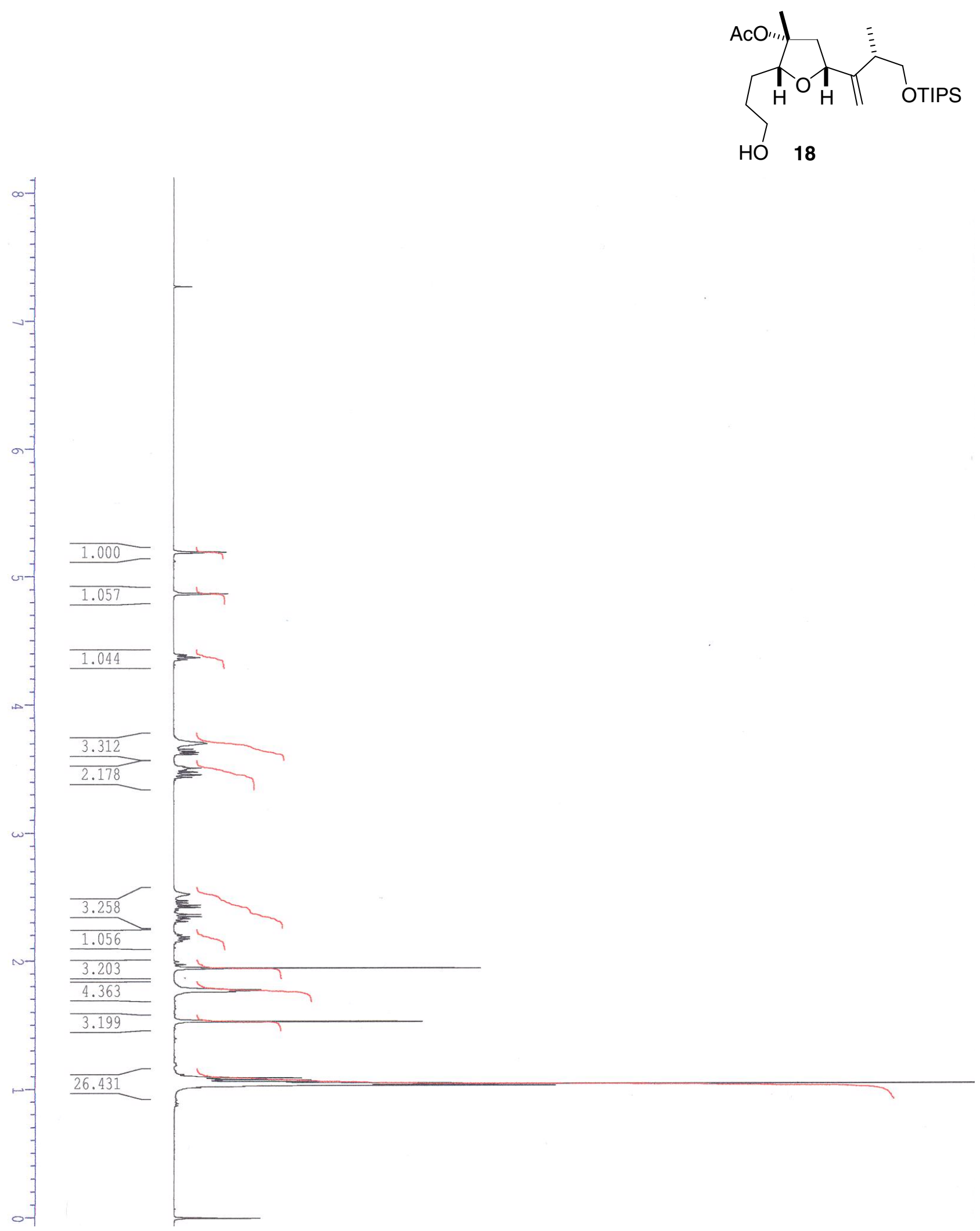


\section{J. S. Clark et al. - Supporting Information}
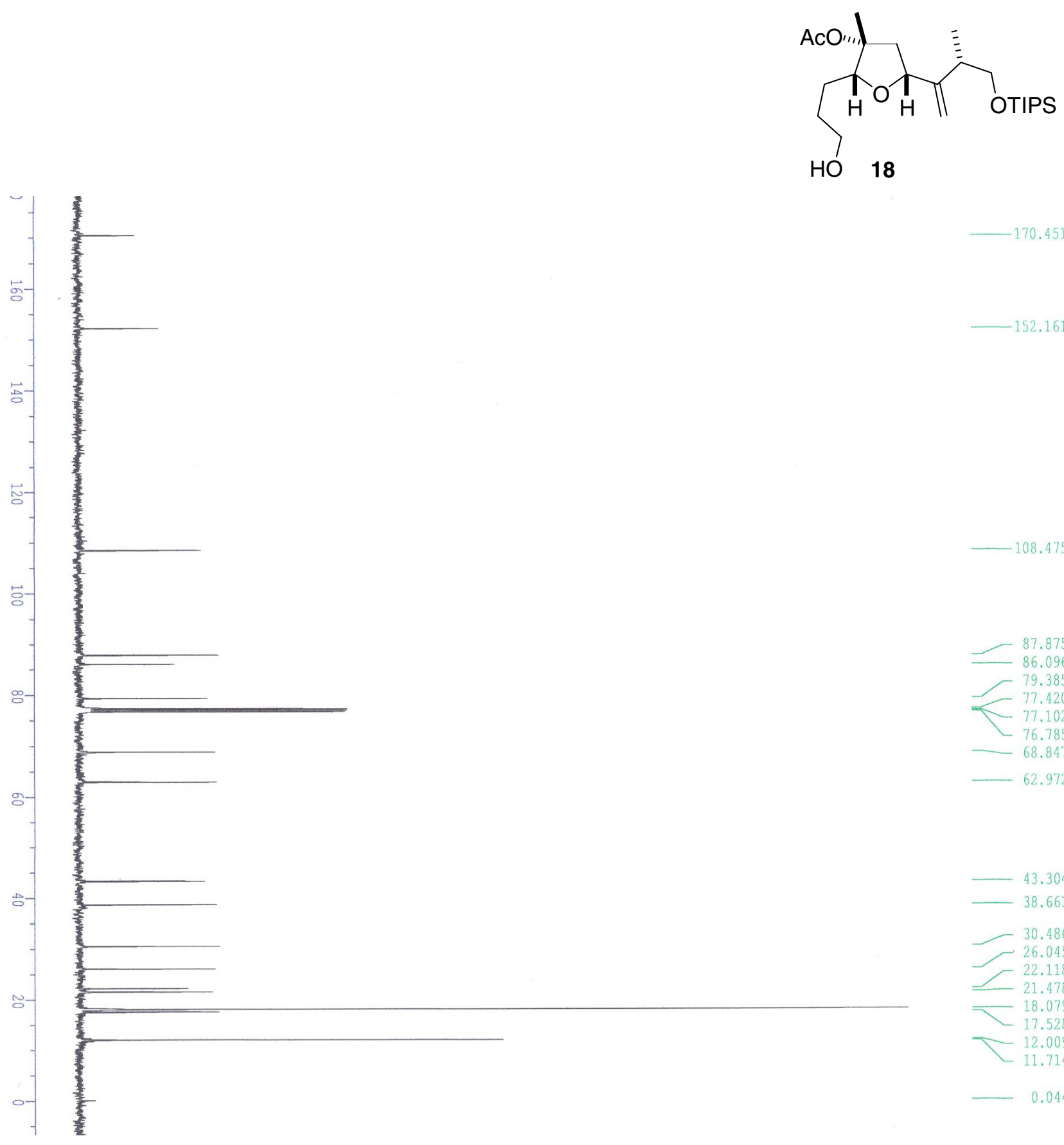


\section{J. S. Clark et al. - Supporting Information}
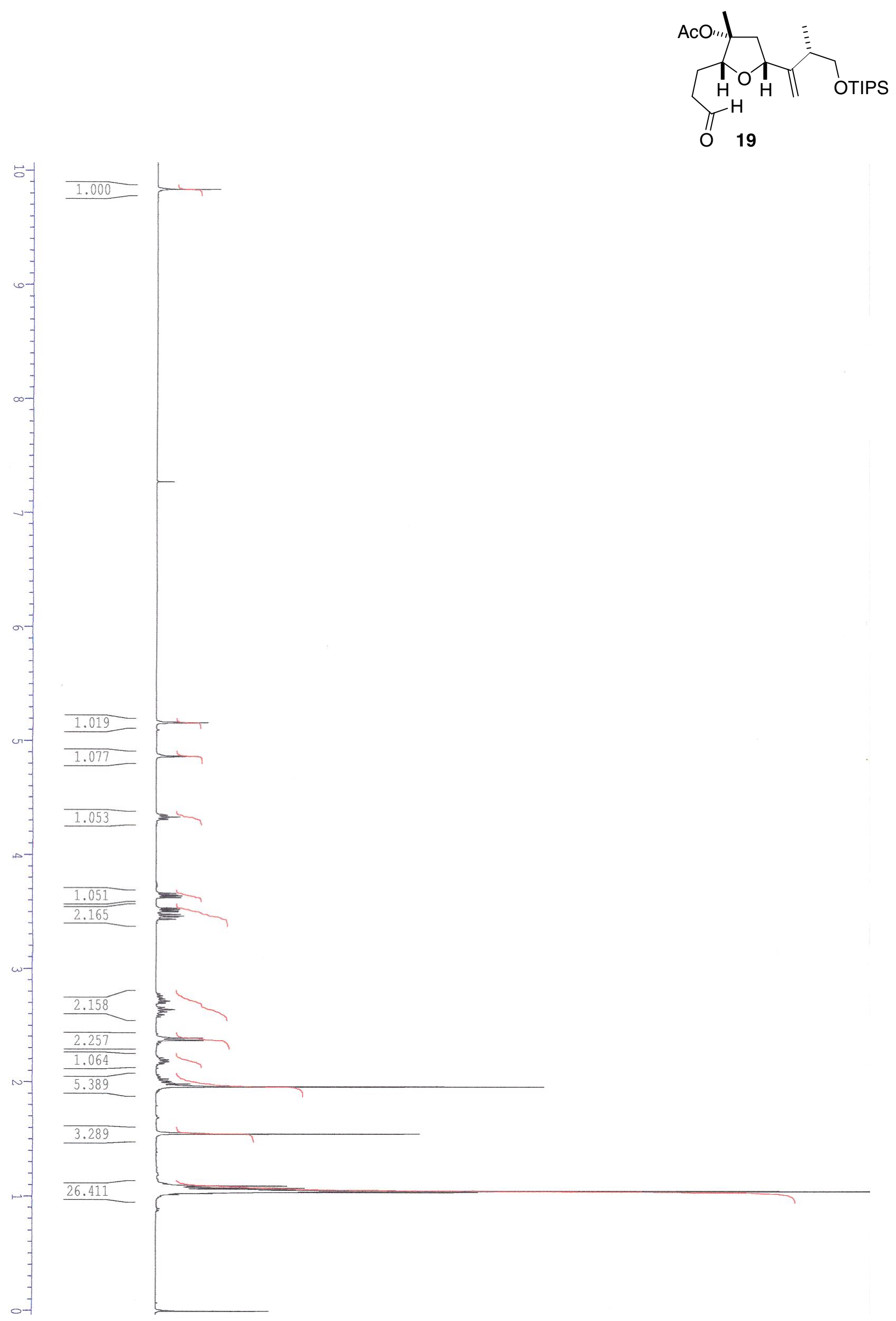


\section{J. S. Clark et al. - Supporting Information}
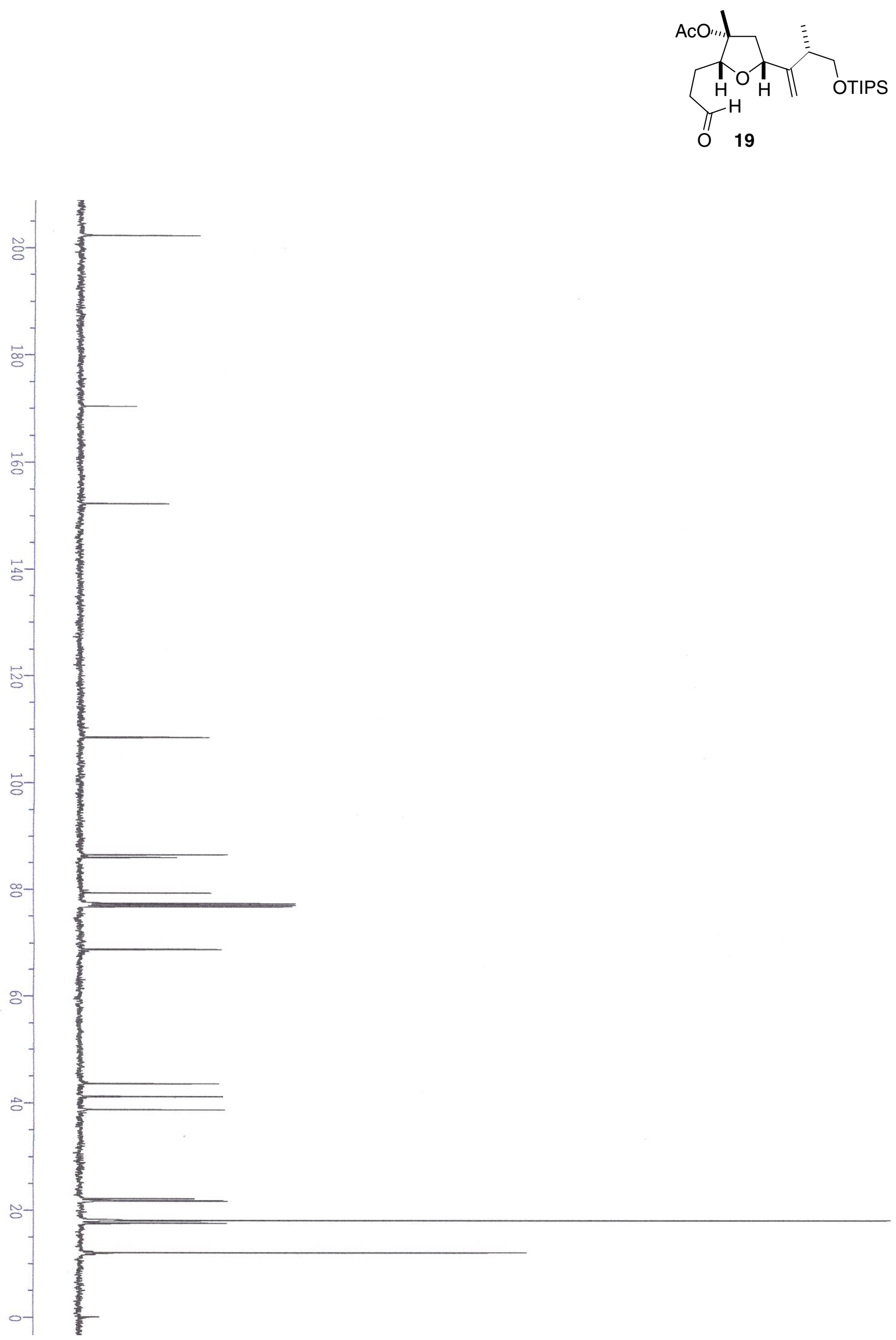
J. S. Clark et al. - Supporting Information
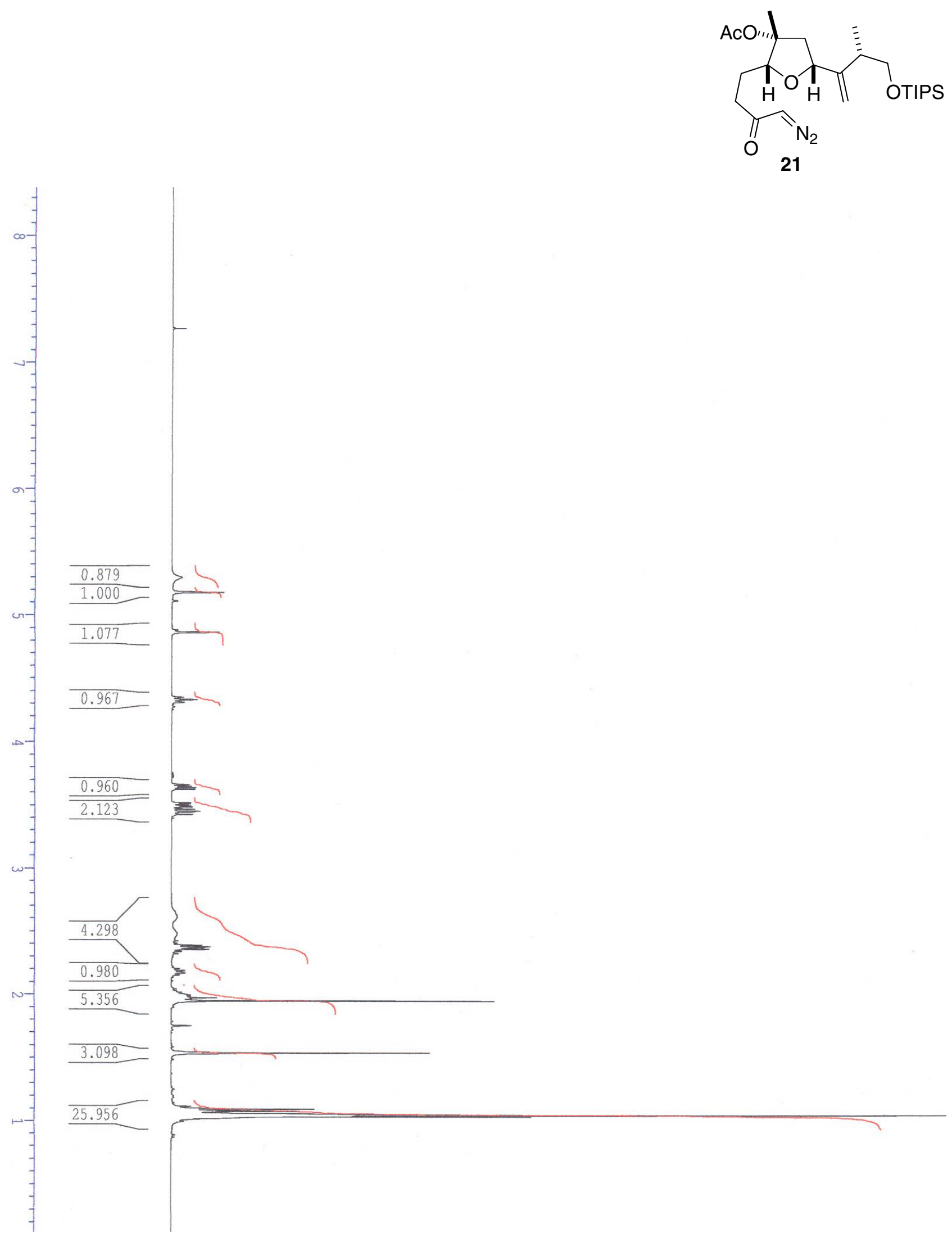
J. S. Clark et al. - Supporting Information
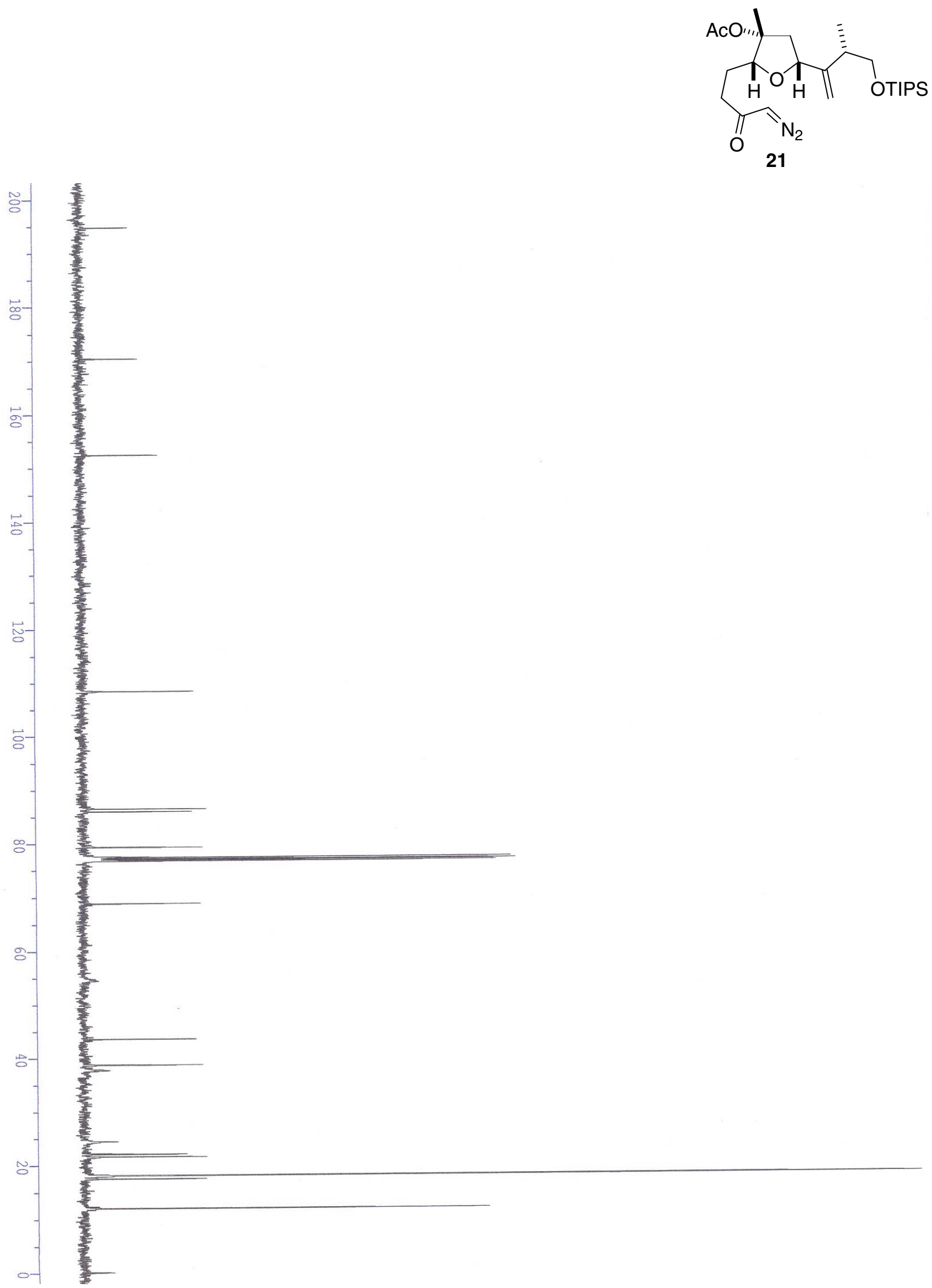

S26 


\section{J. S. Clark et al. - Supporting Information}

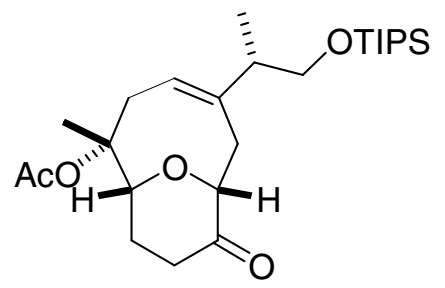

23

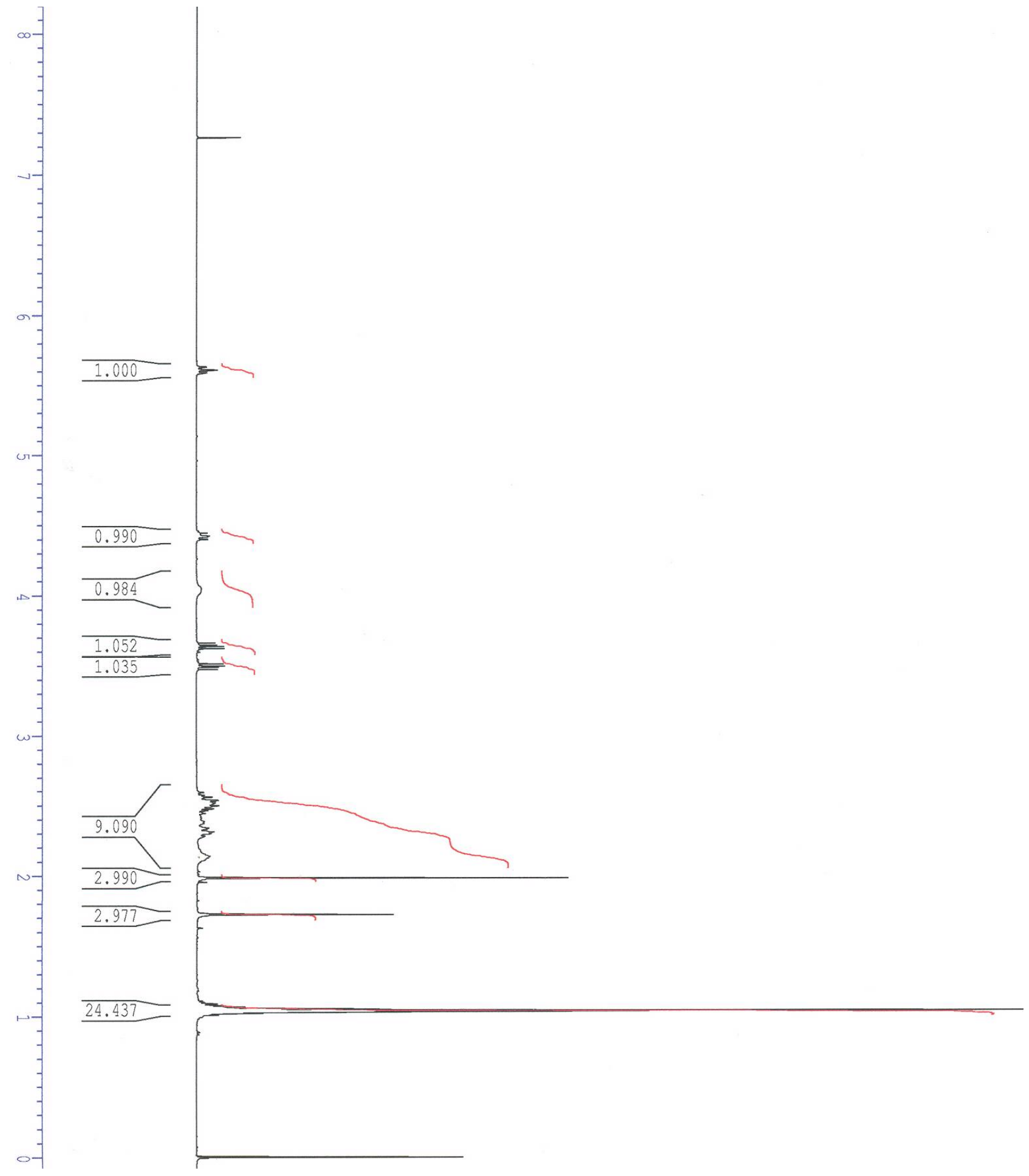




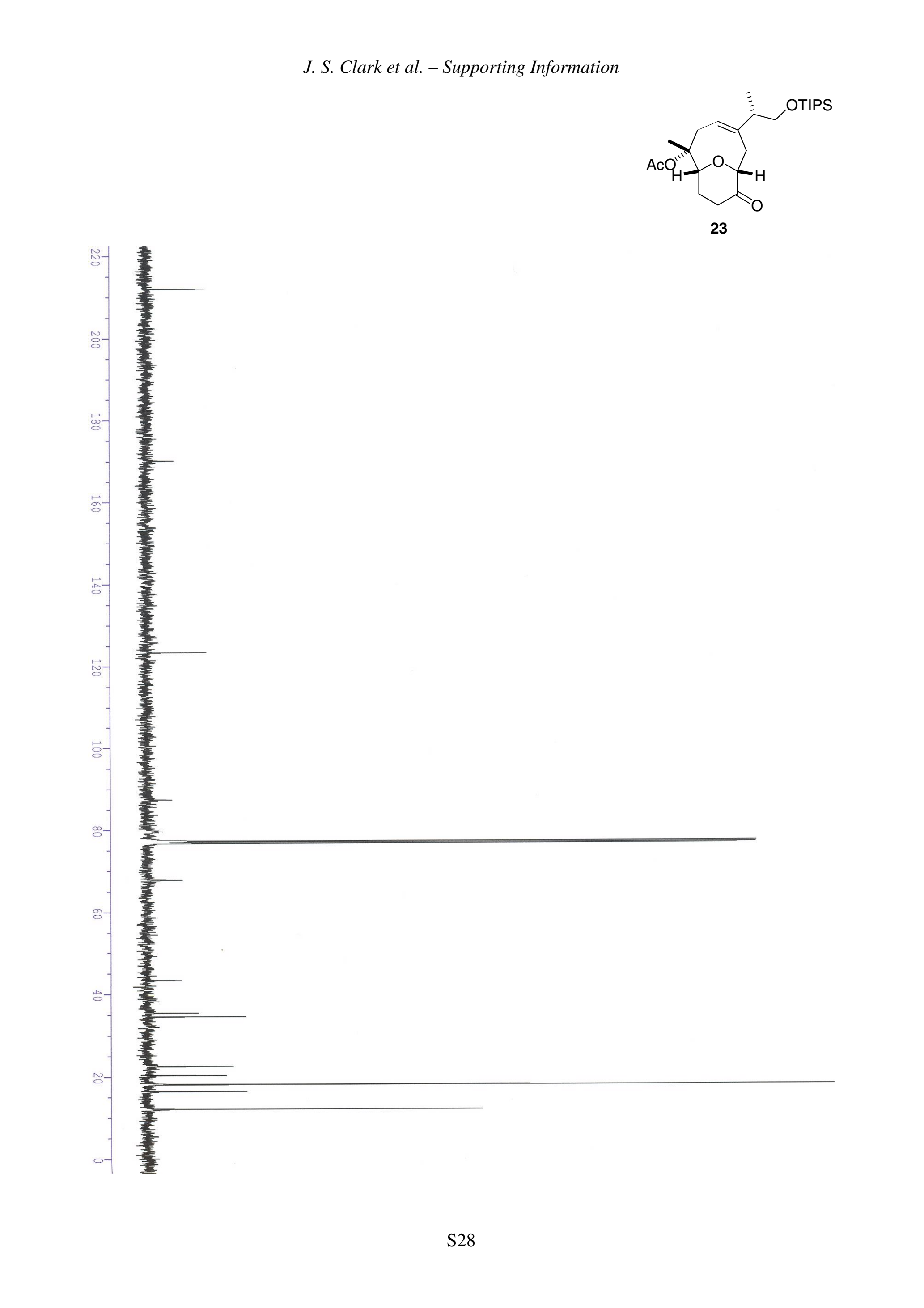




\section{J. S. Clark et al. - Supporting Information}

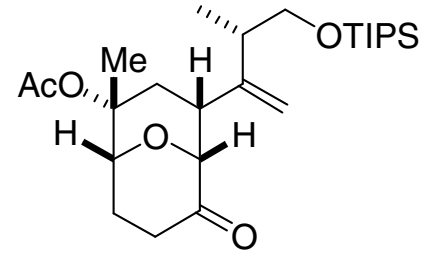

24

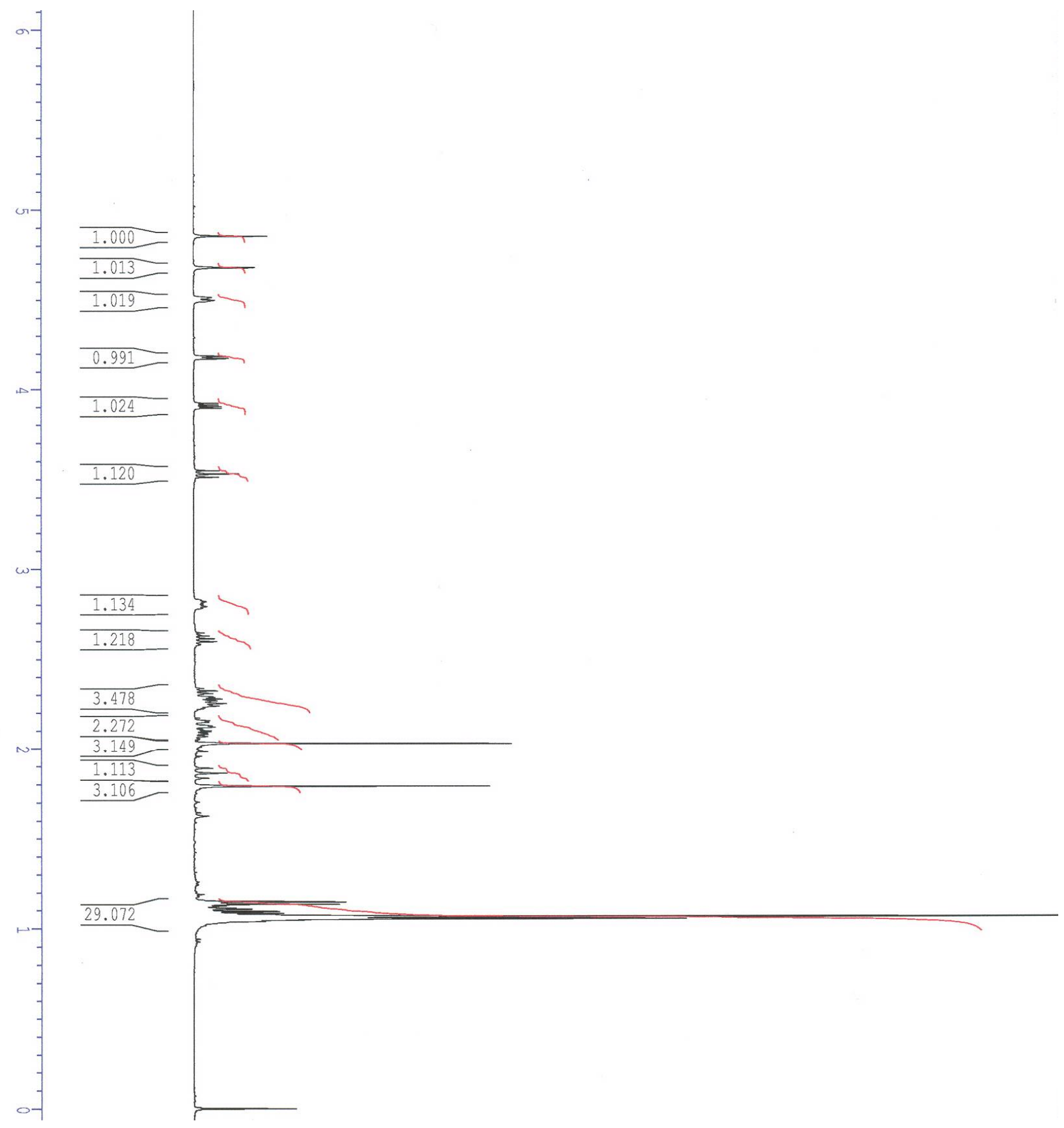




\section{J. S. Clark et al. - Supporting Information}

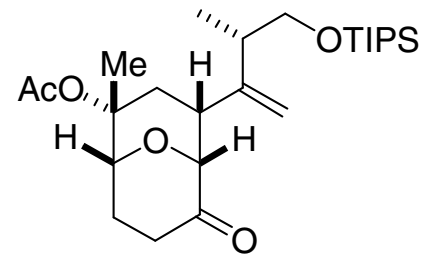

24

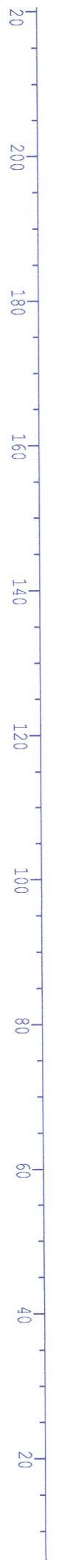

政
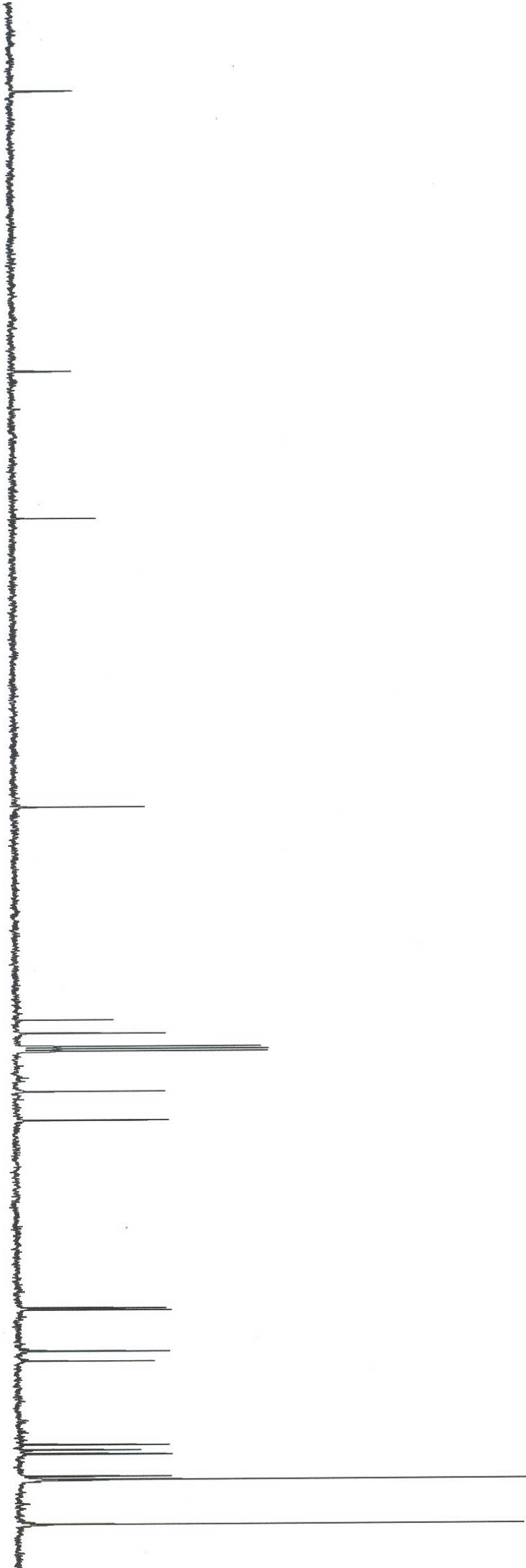

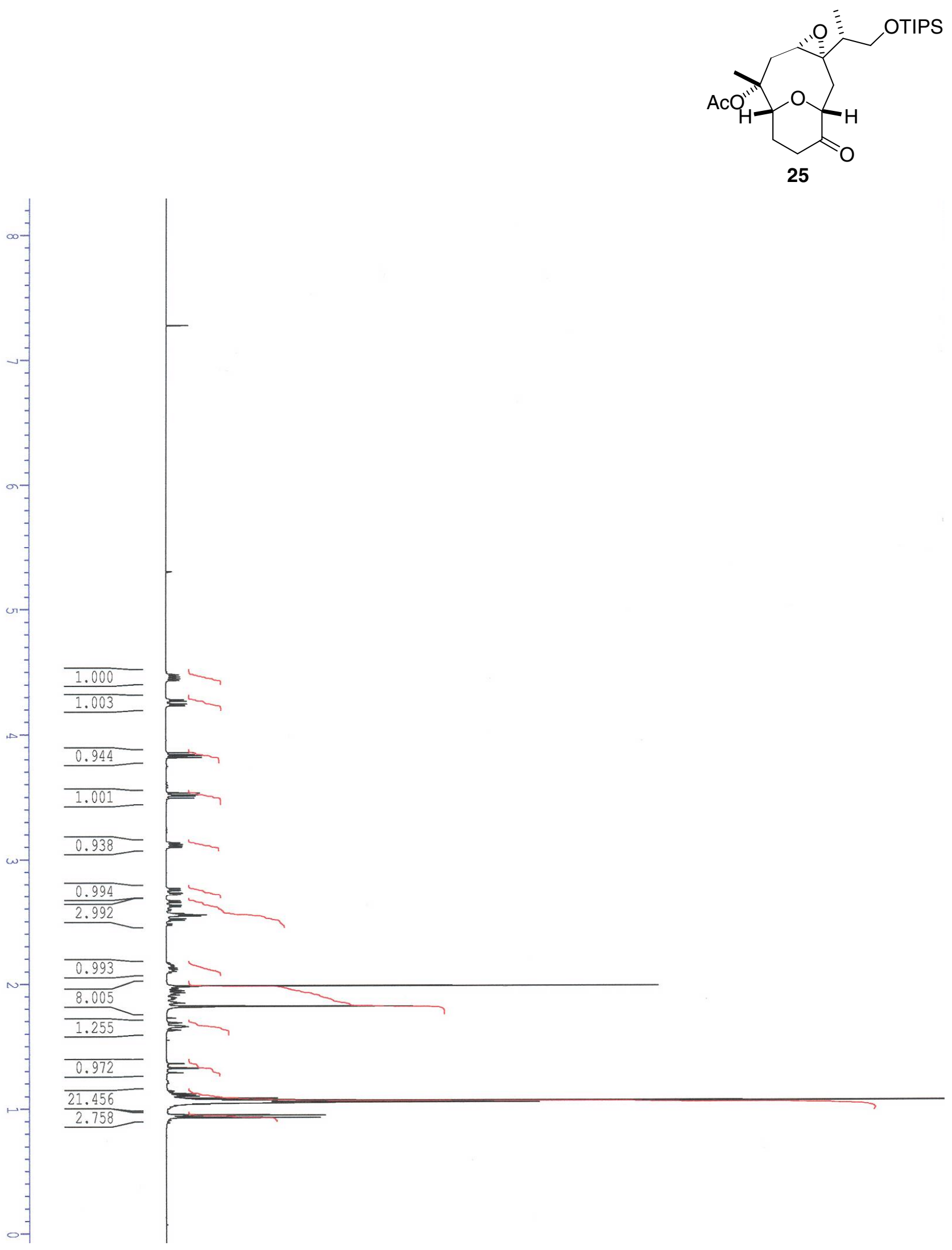


\section{J. S. Clark et al. - Supporting Information}

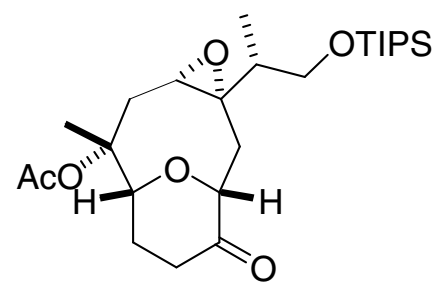

25

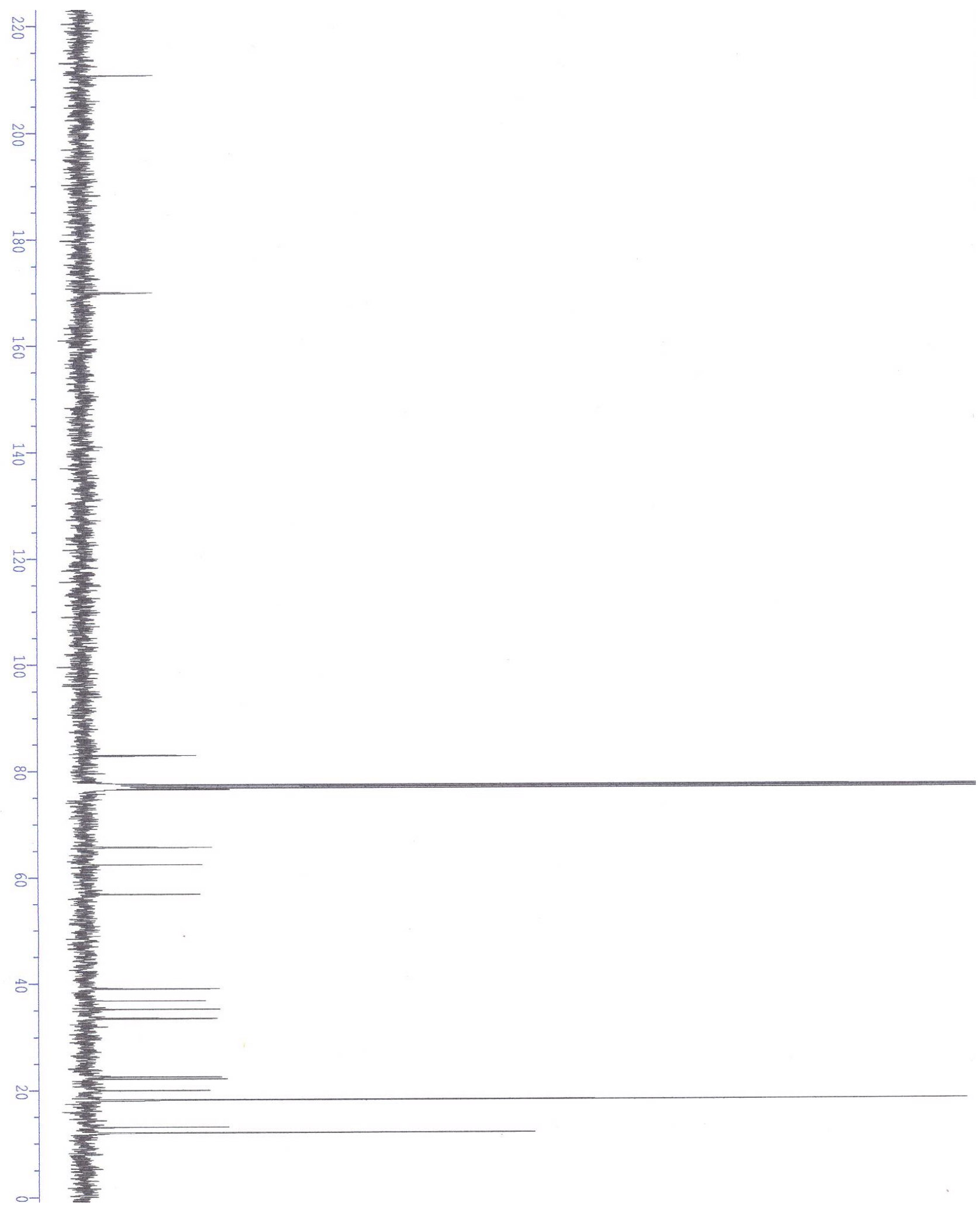




\section{J. S. Clark et al. - Supporting Information}

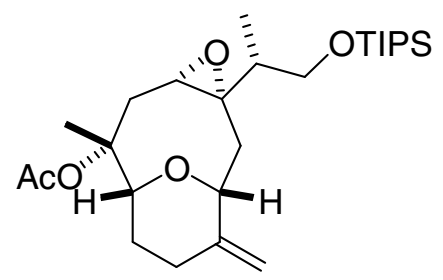

26

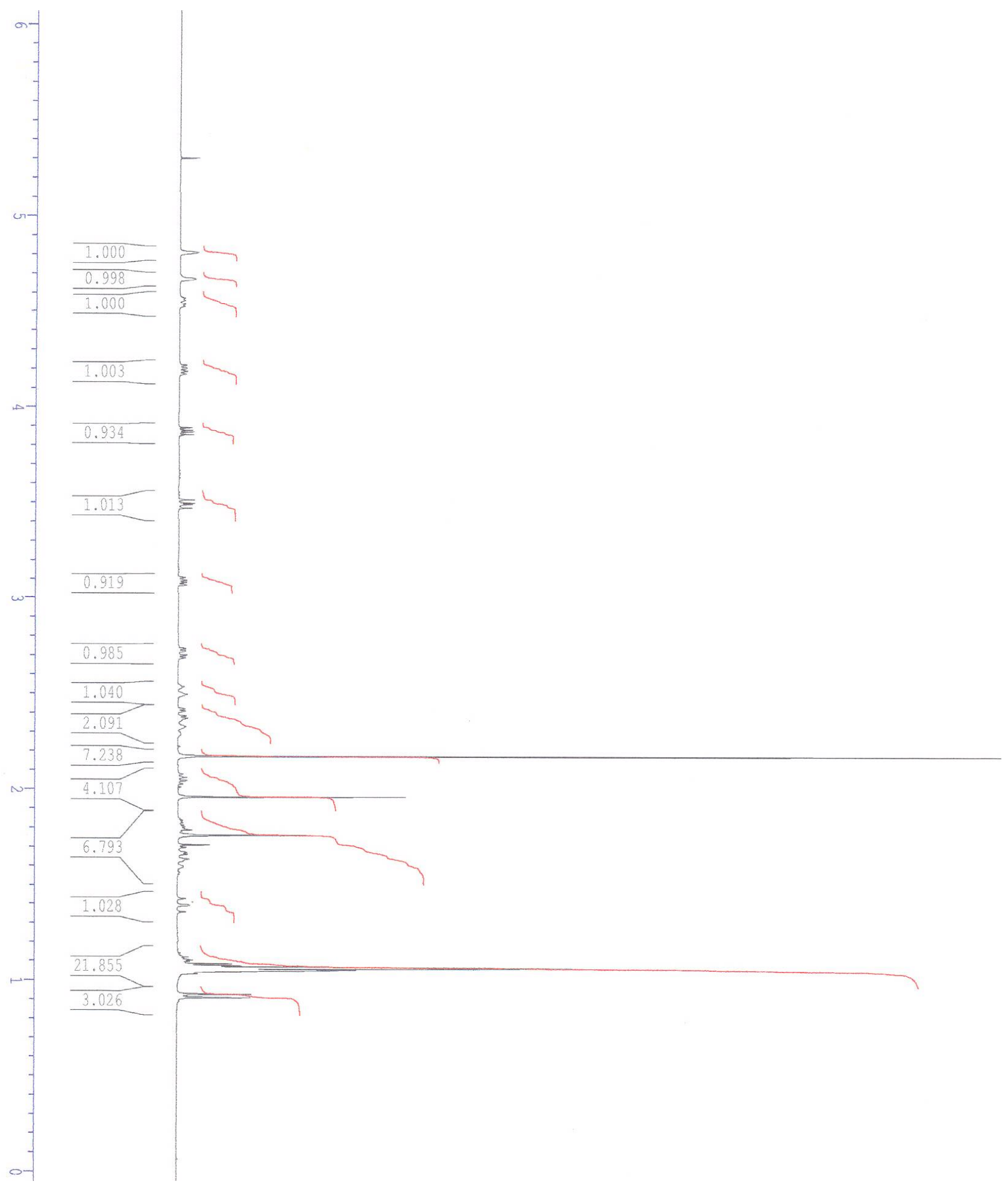




\section{J. S. Clark et al. - Supporting Information}
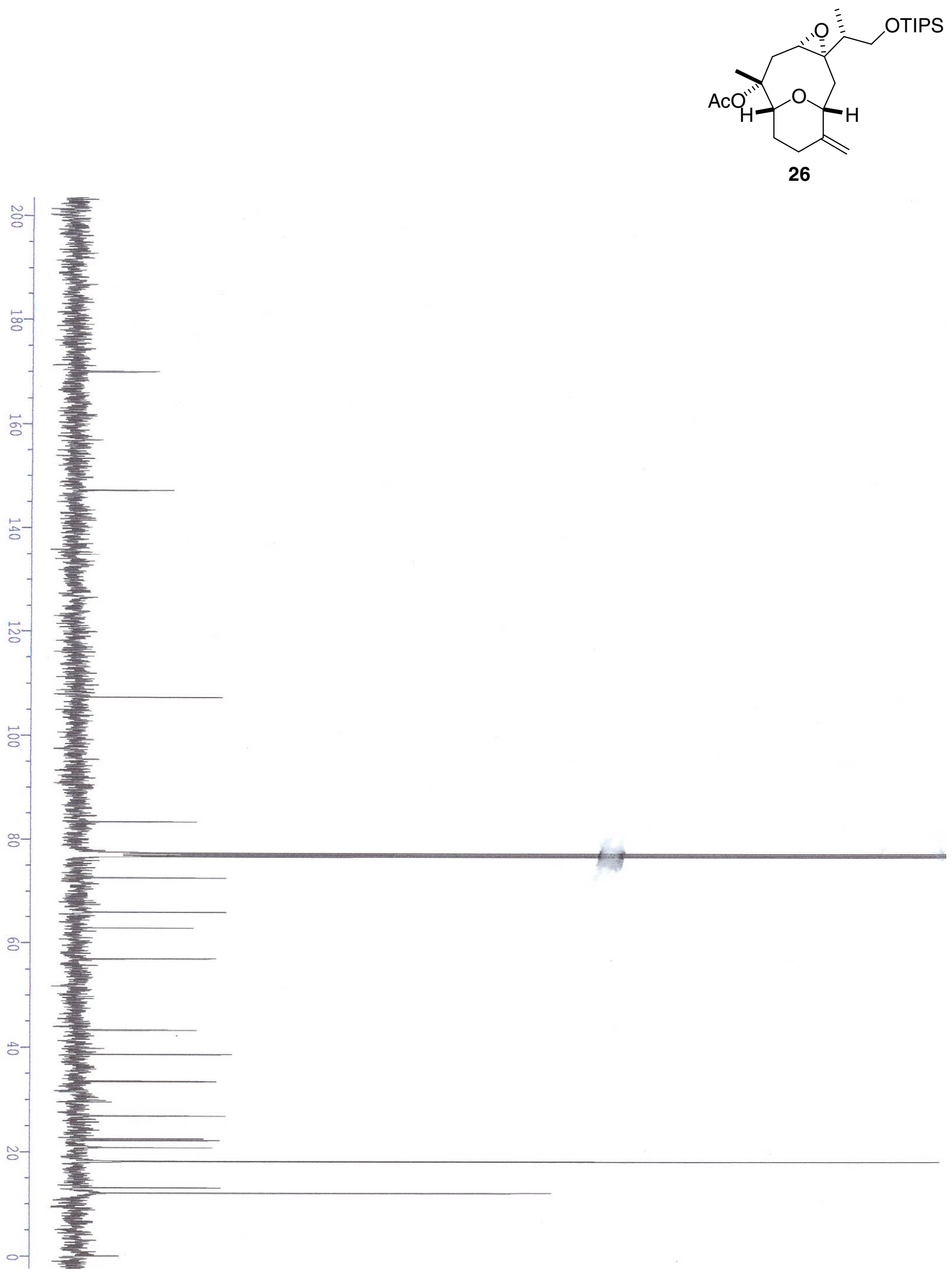


\section{J. S. Clark et al. - Supporting Information}

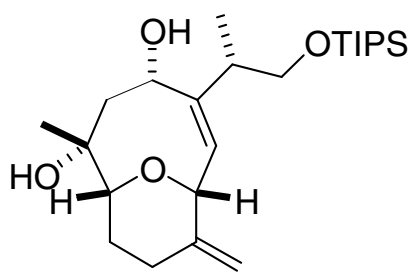

27

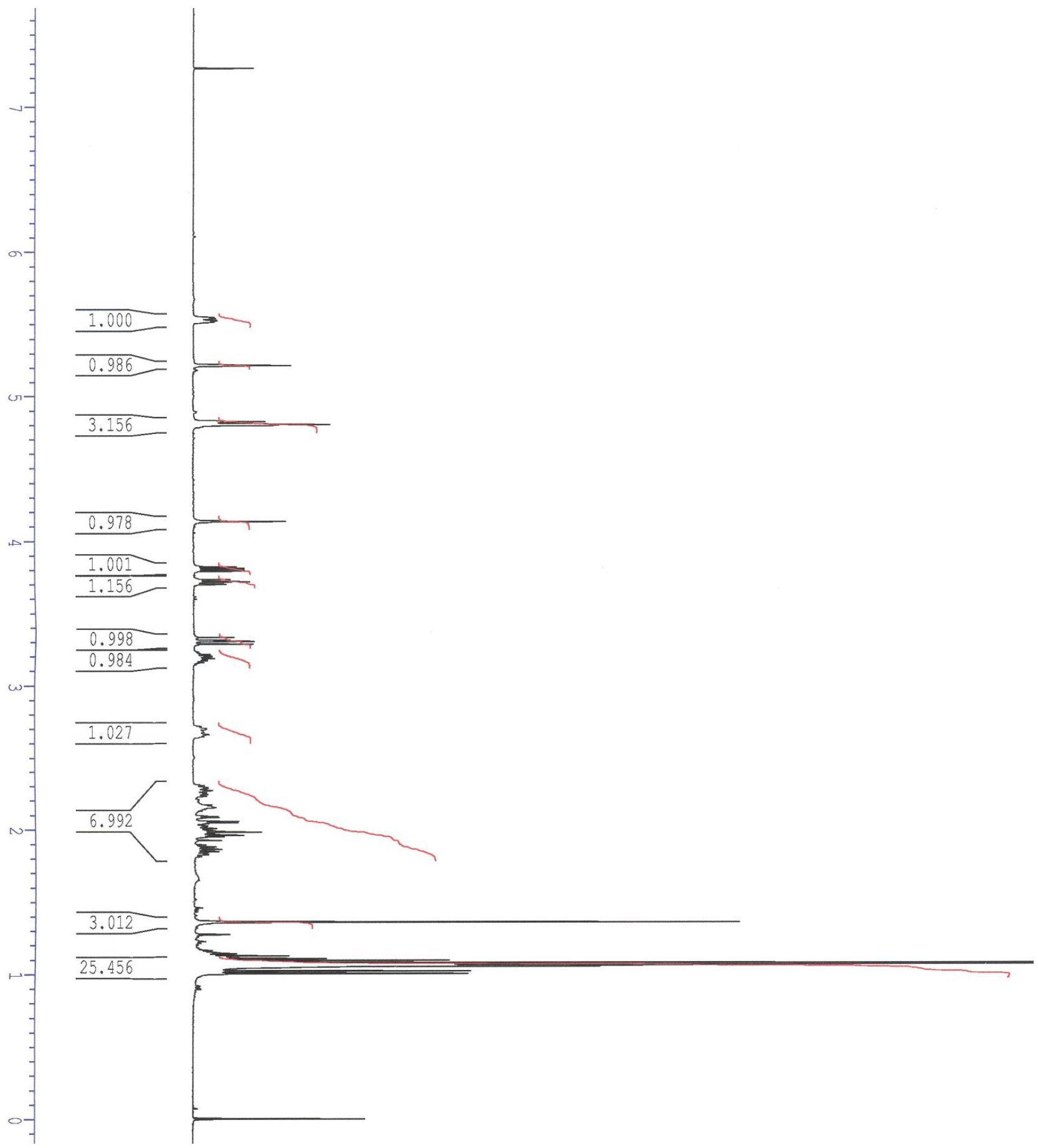




\section{J. S. Clark et al. - Supporting Information}<smiles>C=C1CCC2(O)OC1C=C([C@H](C)CO[PH3+])[C@H](O)CC2(C)C</smiles>

27

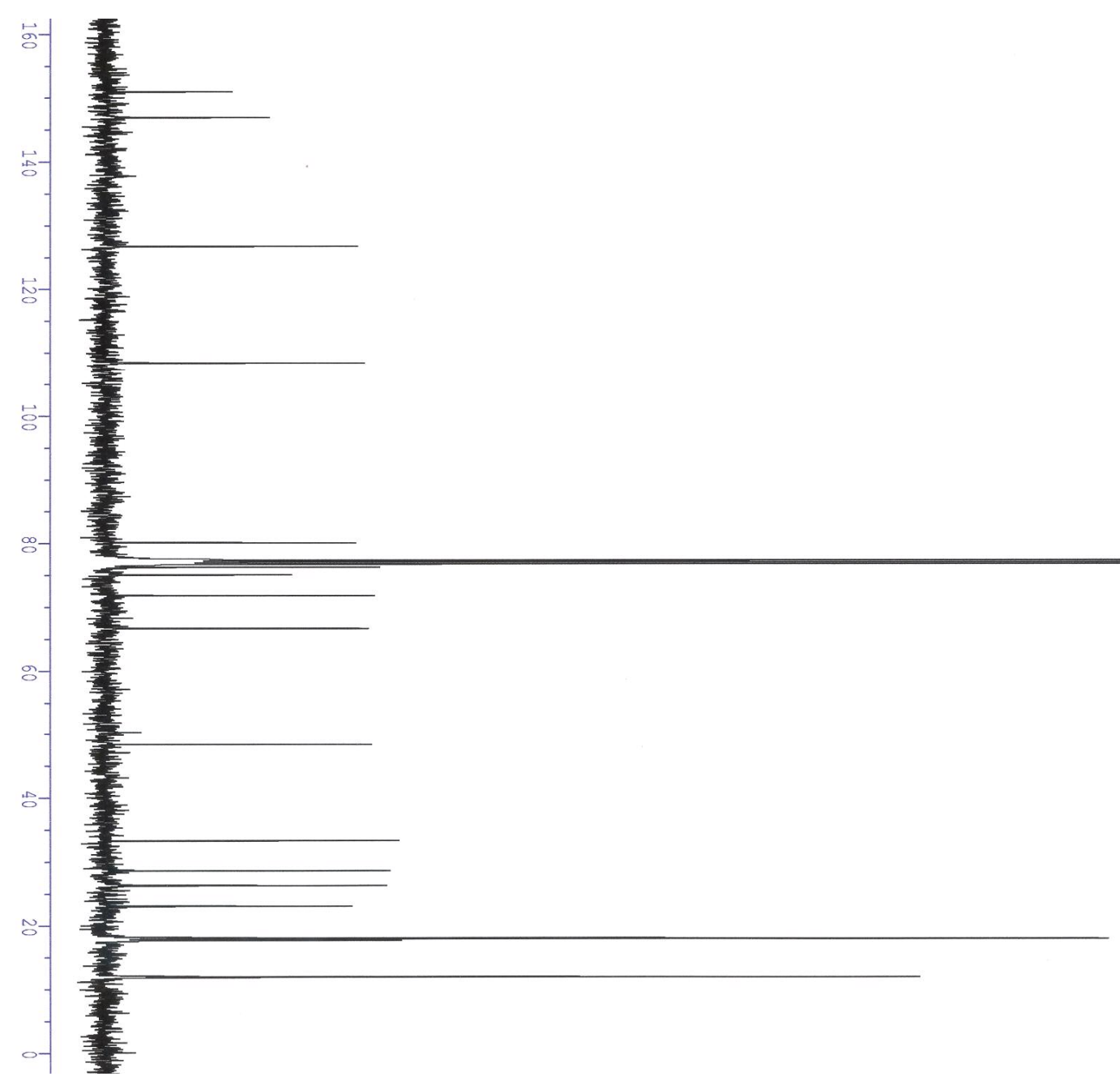




\section{J. S. Clark et al. - Supporting Information}

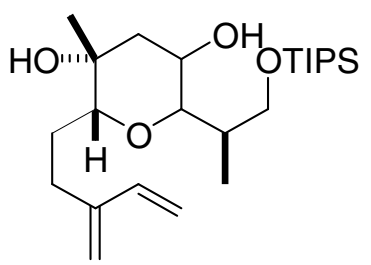

28

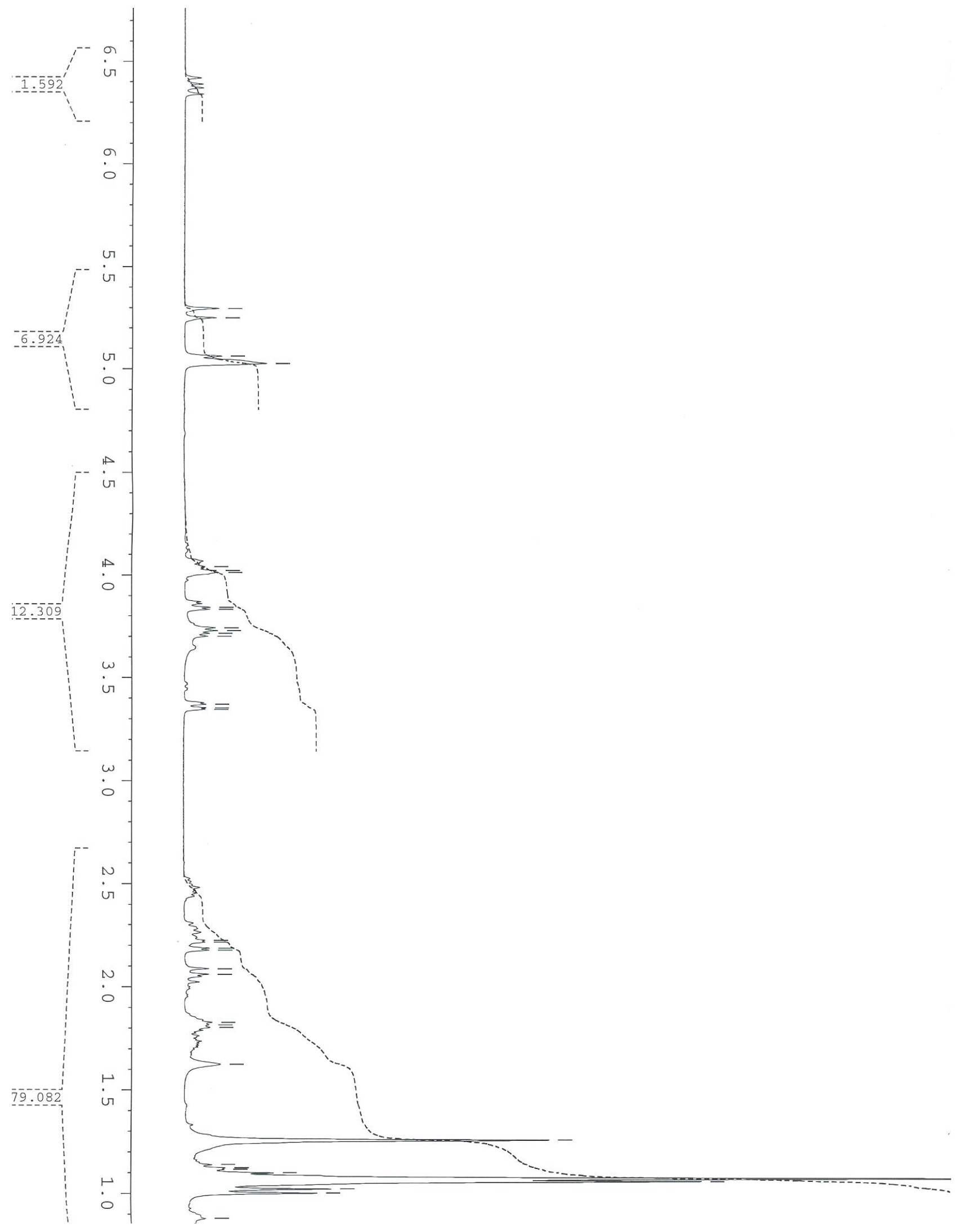


J. S. Clark et al. - Supporting Information

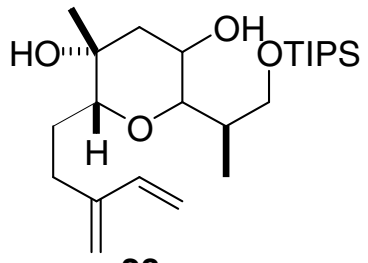

28

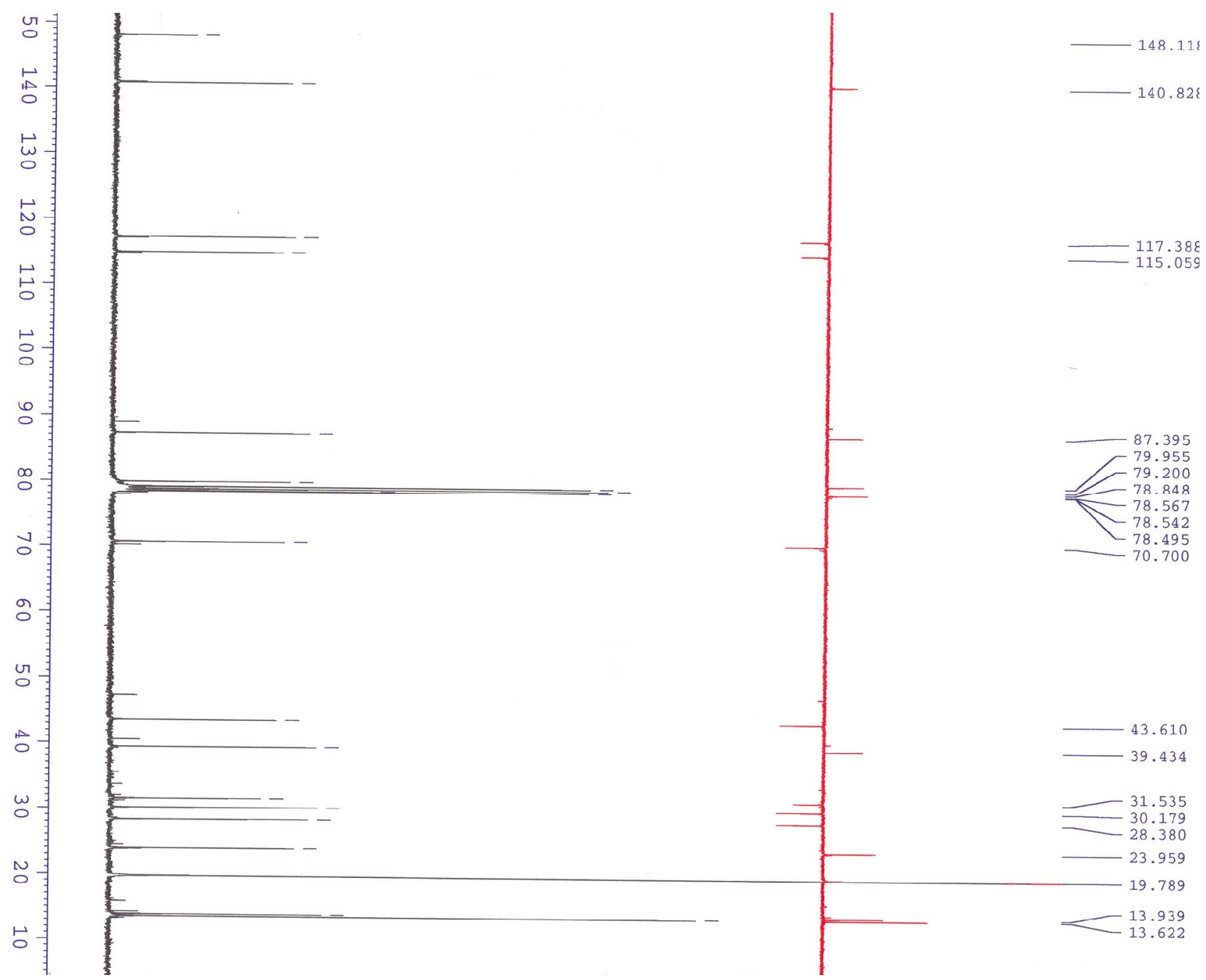




\section{J. S. Clark et al. - Supporting Information}

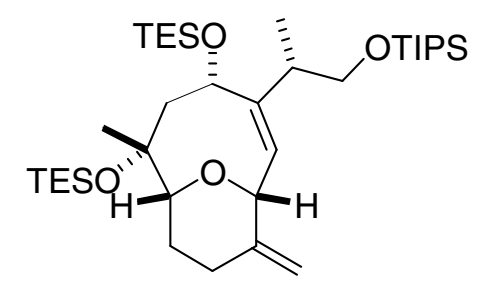

29

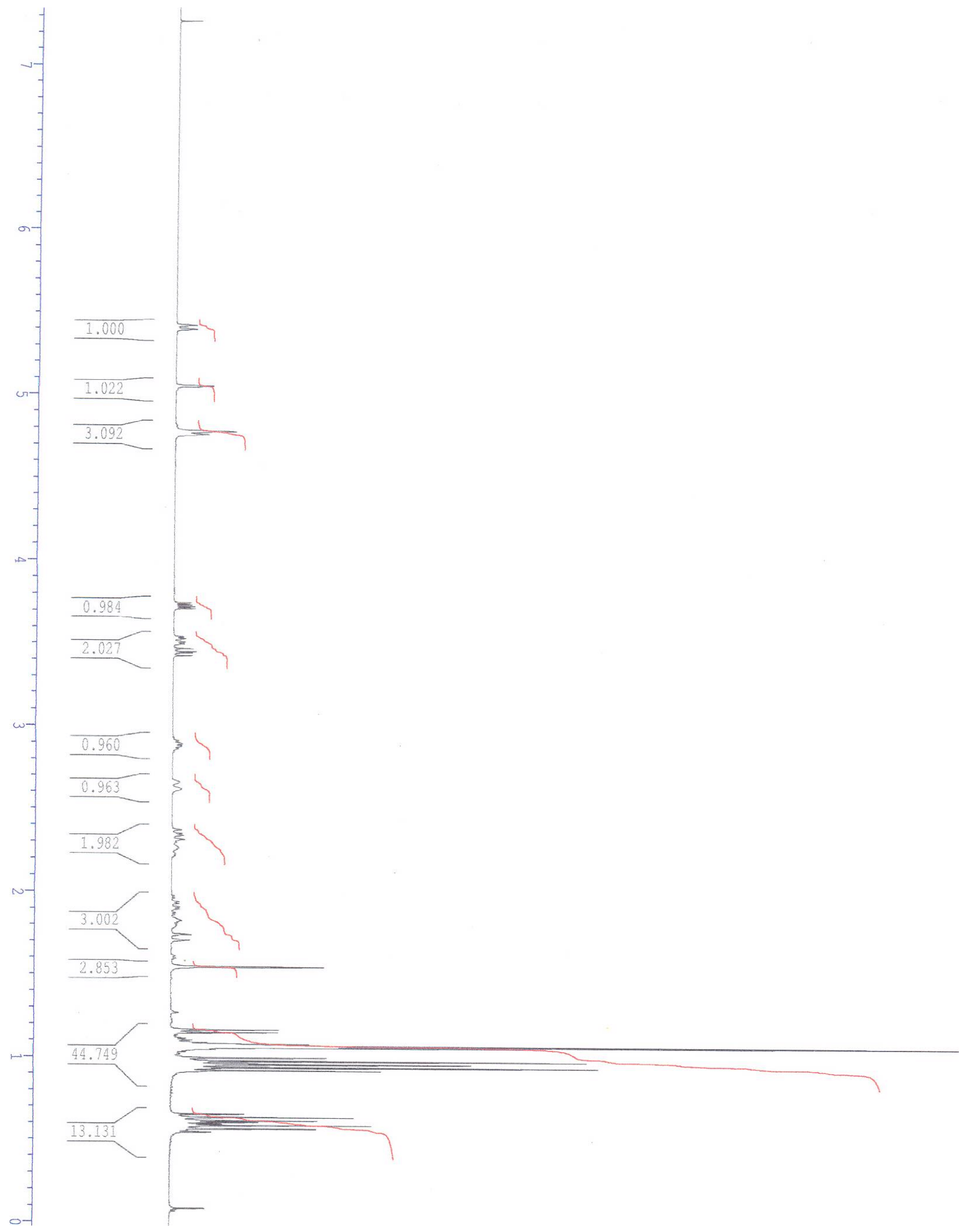


J. S. Clark et al. - Supporting Information

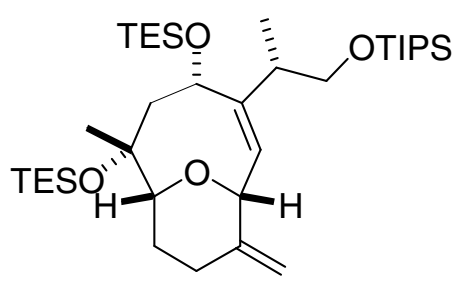

29

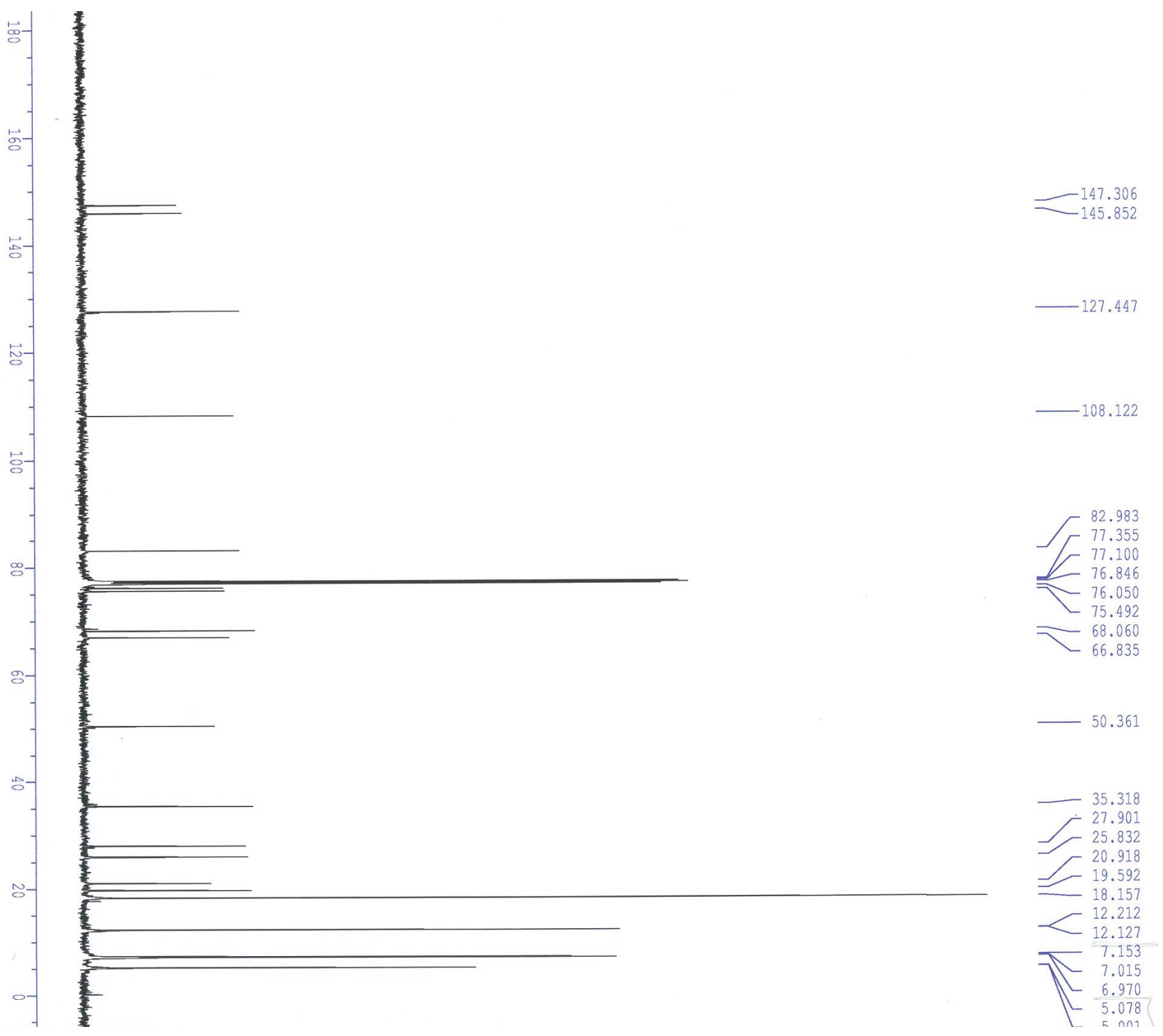


J. S. Clark et al. - Supporting Information

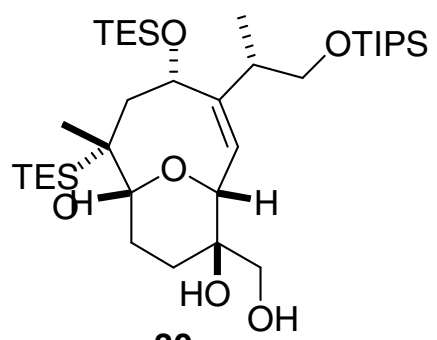

30

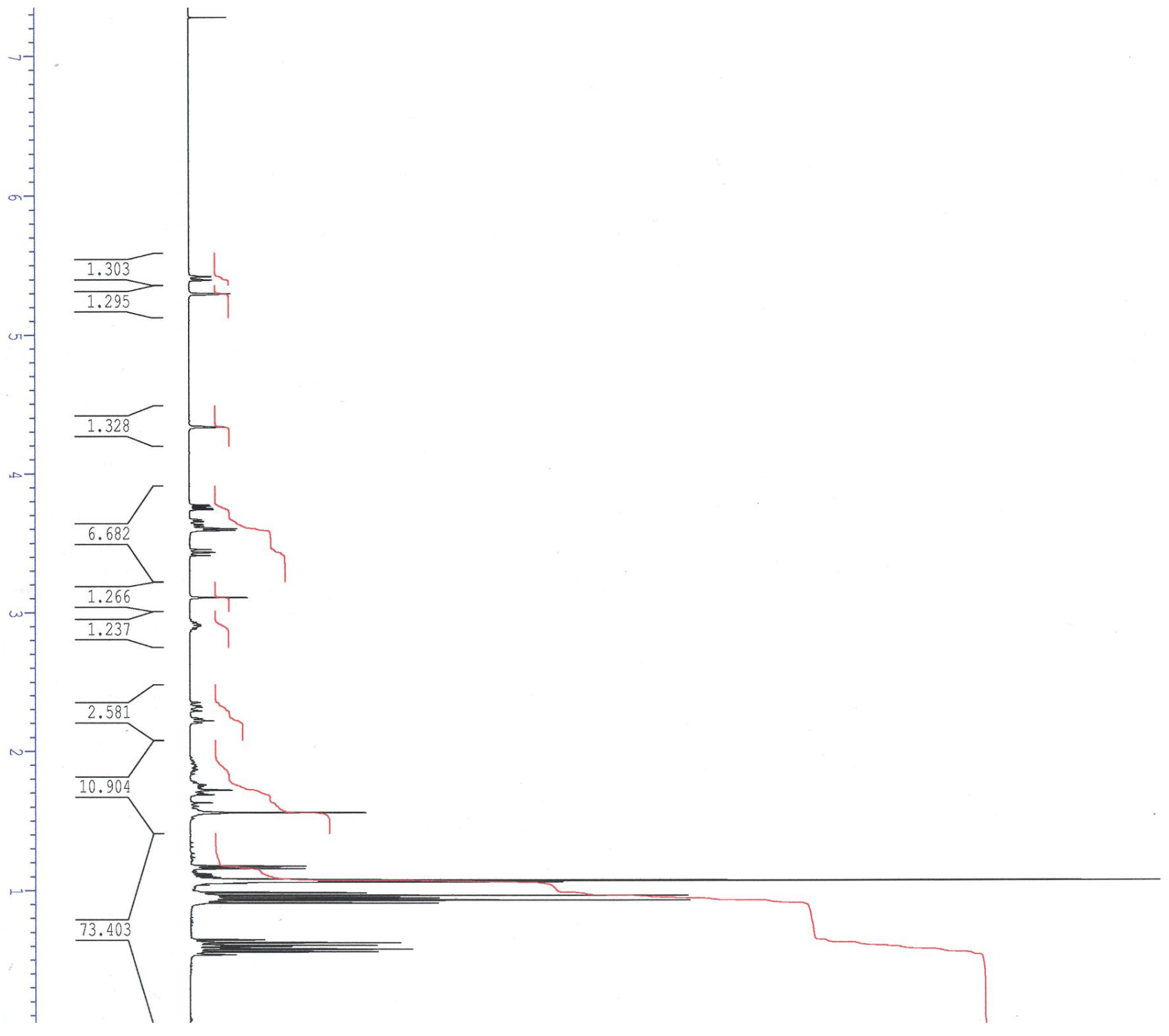


J. S. Clark et al. - Supporting Information
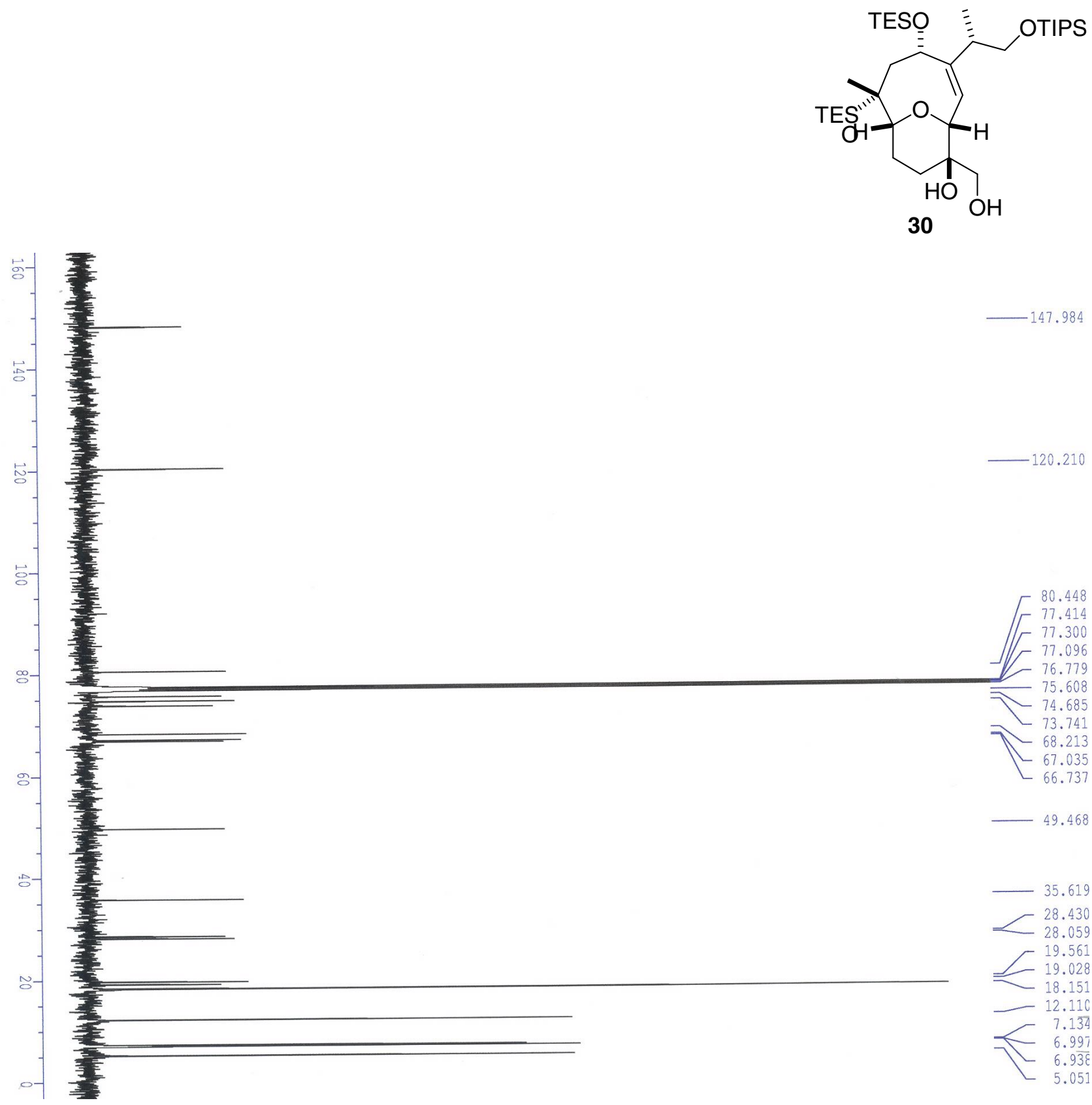

$-147.984$

$\Gamma^{80.448}$

F 77.414

76.779

75.608

773.741

$-68.213$

$-67.035$

49.468

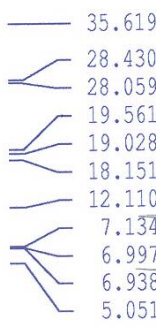


J. S. Clark et al. - Supporting Information

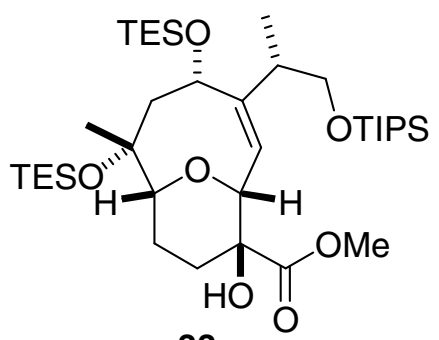

33

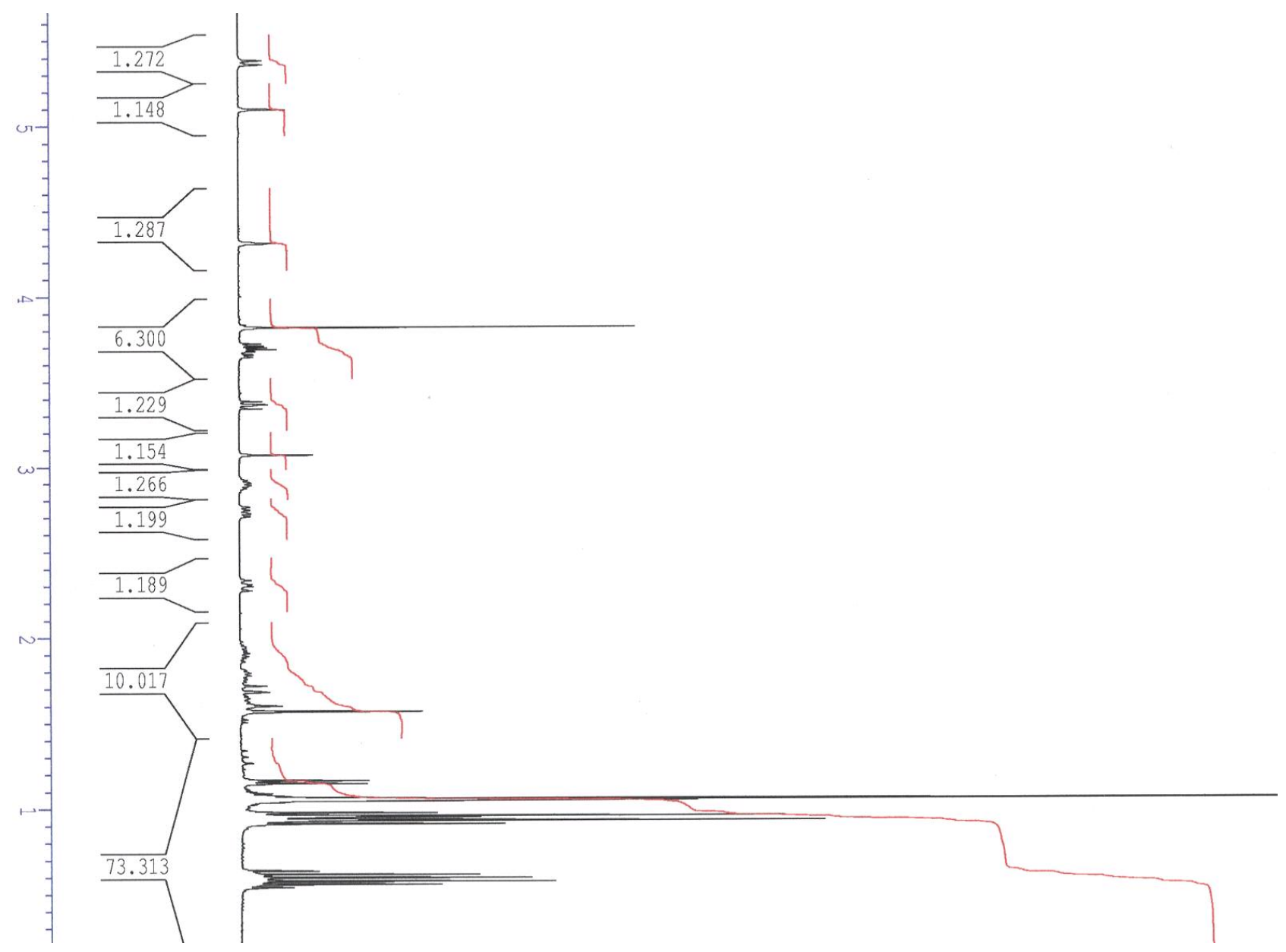




\section{J. S. Clark et al. - Supporting Information}

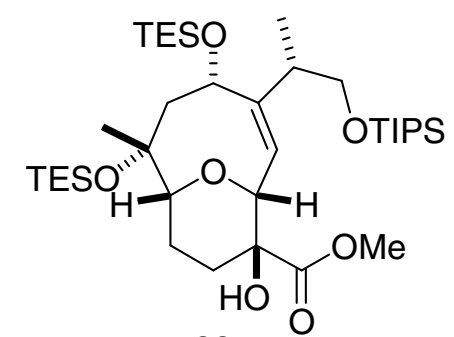

33

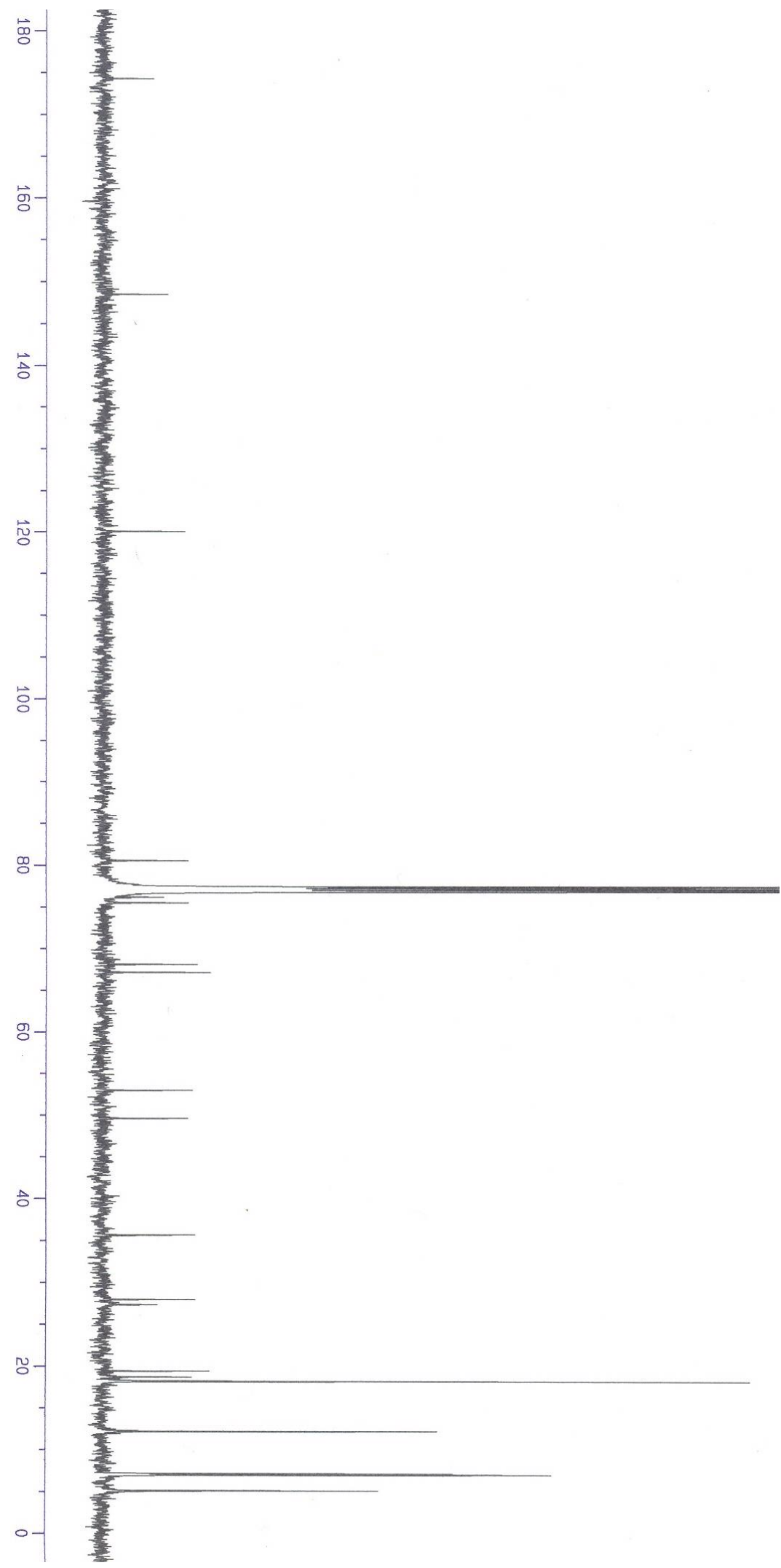


J. S. Clark et al. - Supporting Information

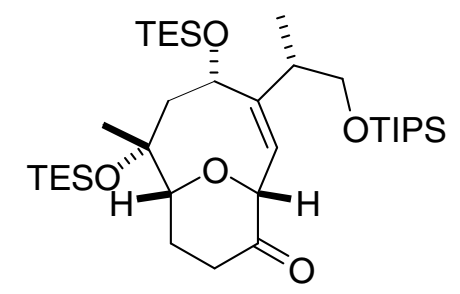

34

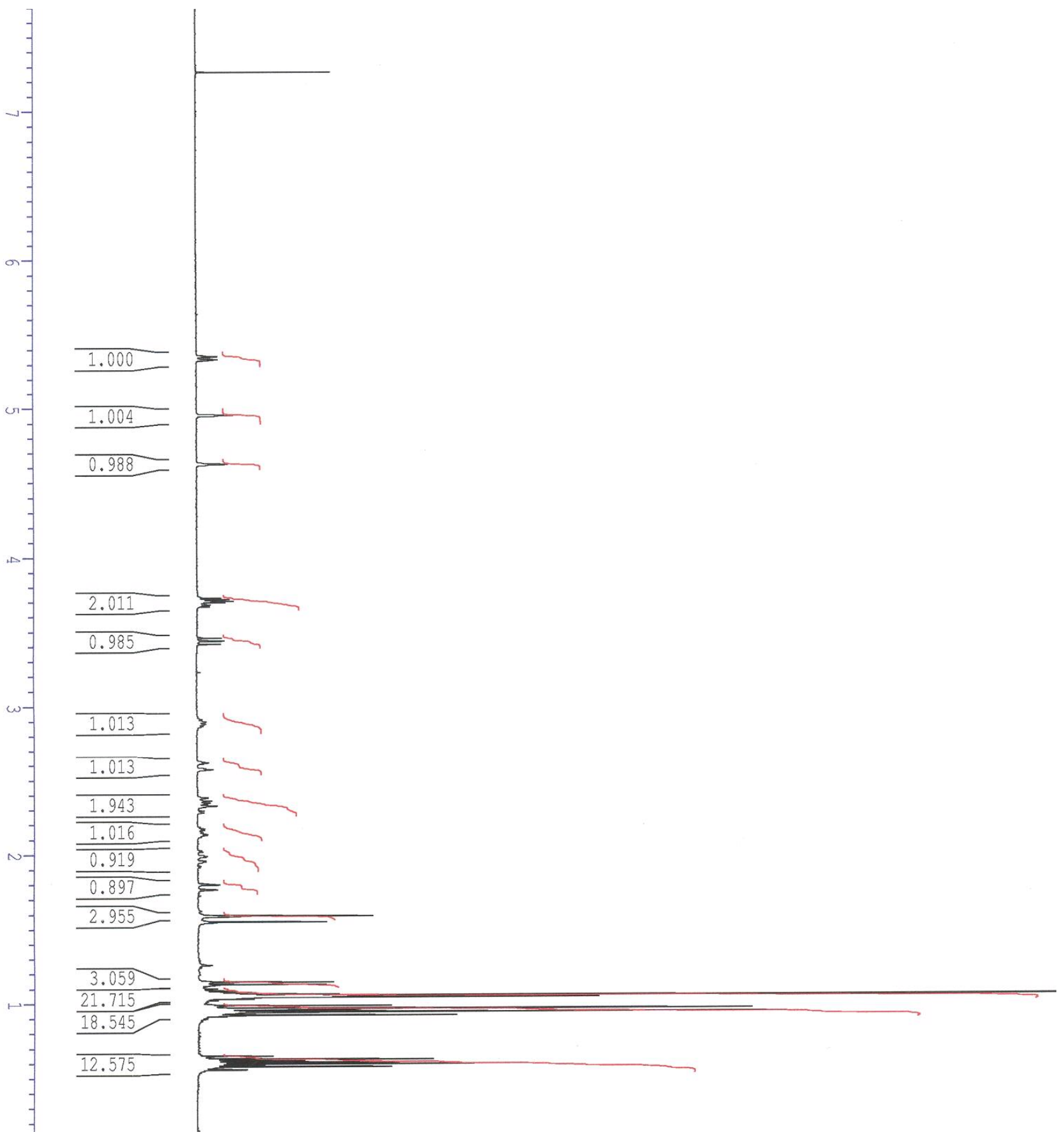


J. S. Clark et al. - Supporting Information

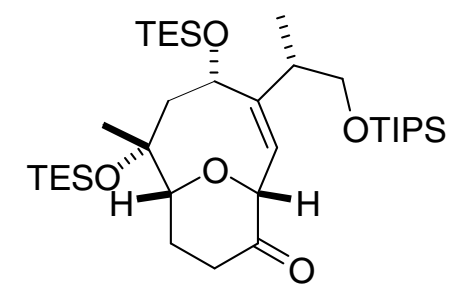

34

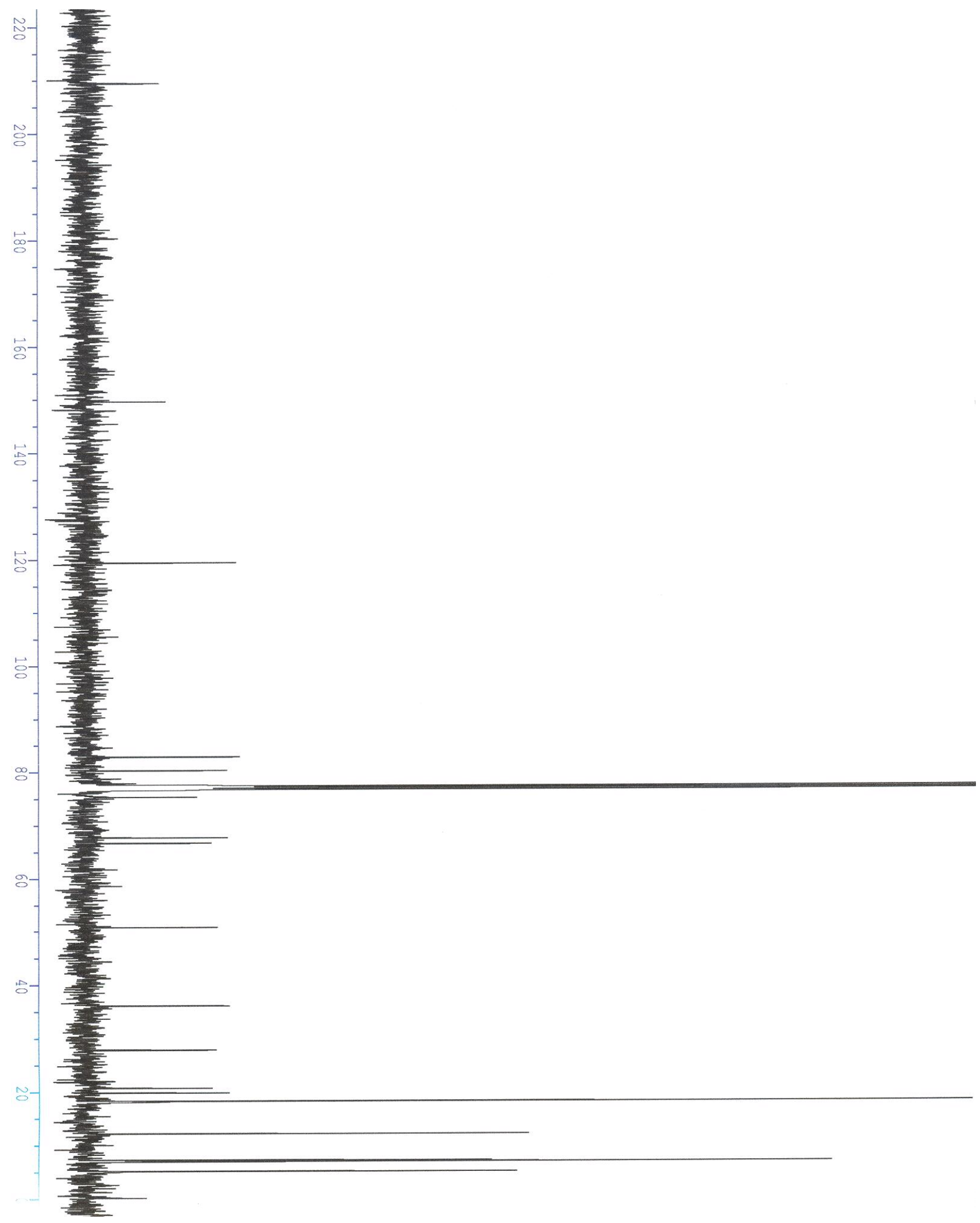




\section{J. S. Clark et al. - Supporting Information}

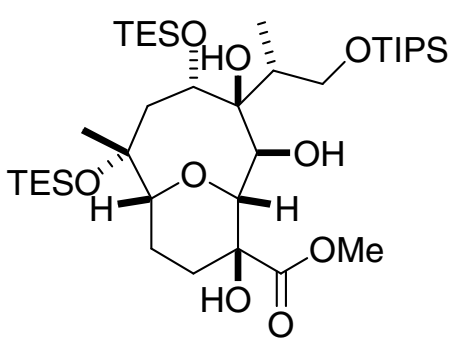

35

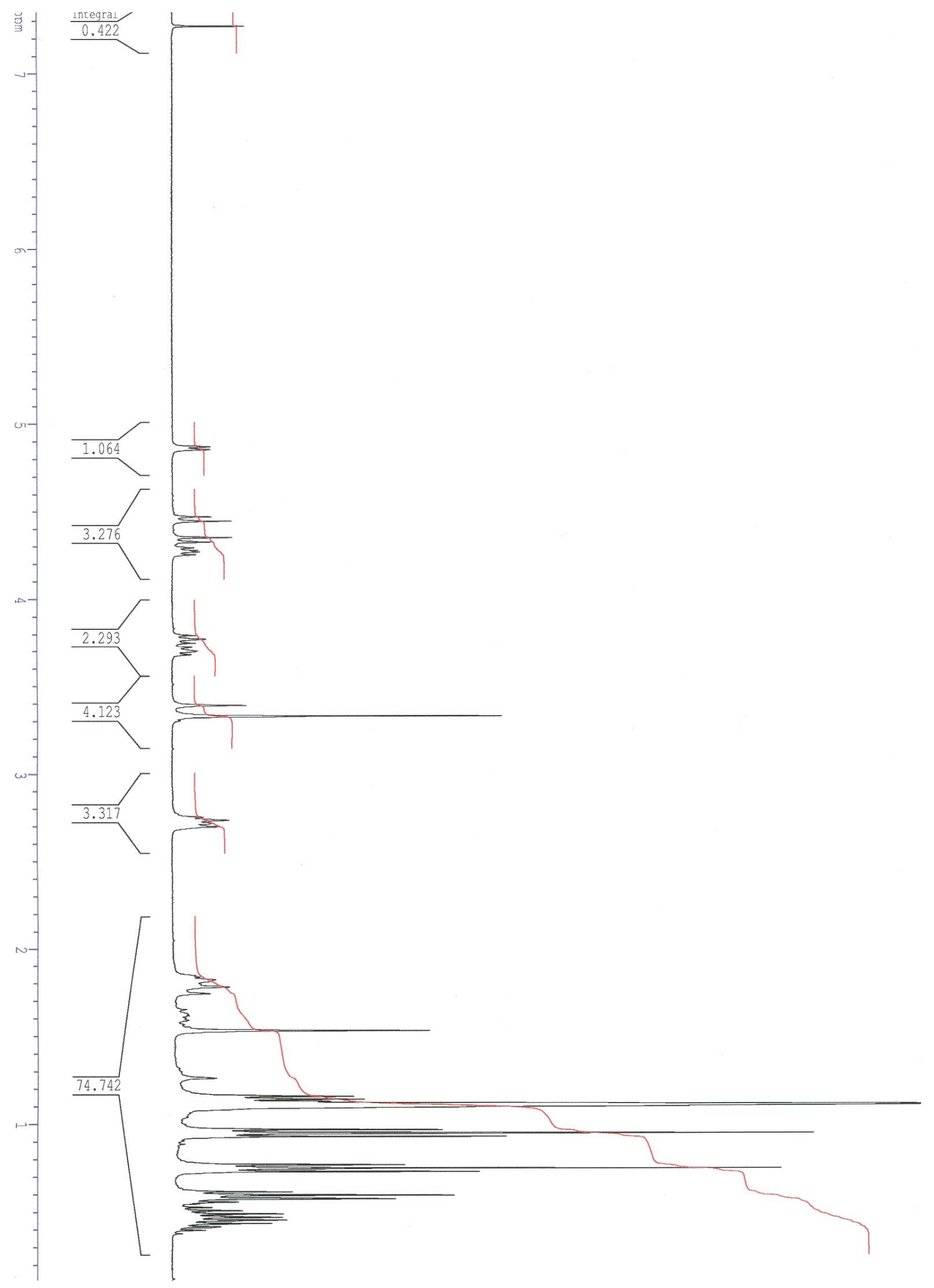


J. S. Clark et al. - Supporting Information

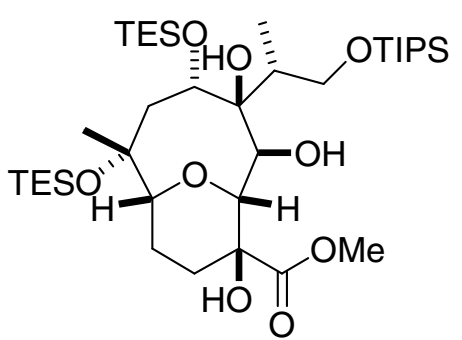

35

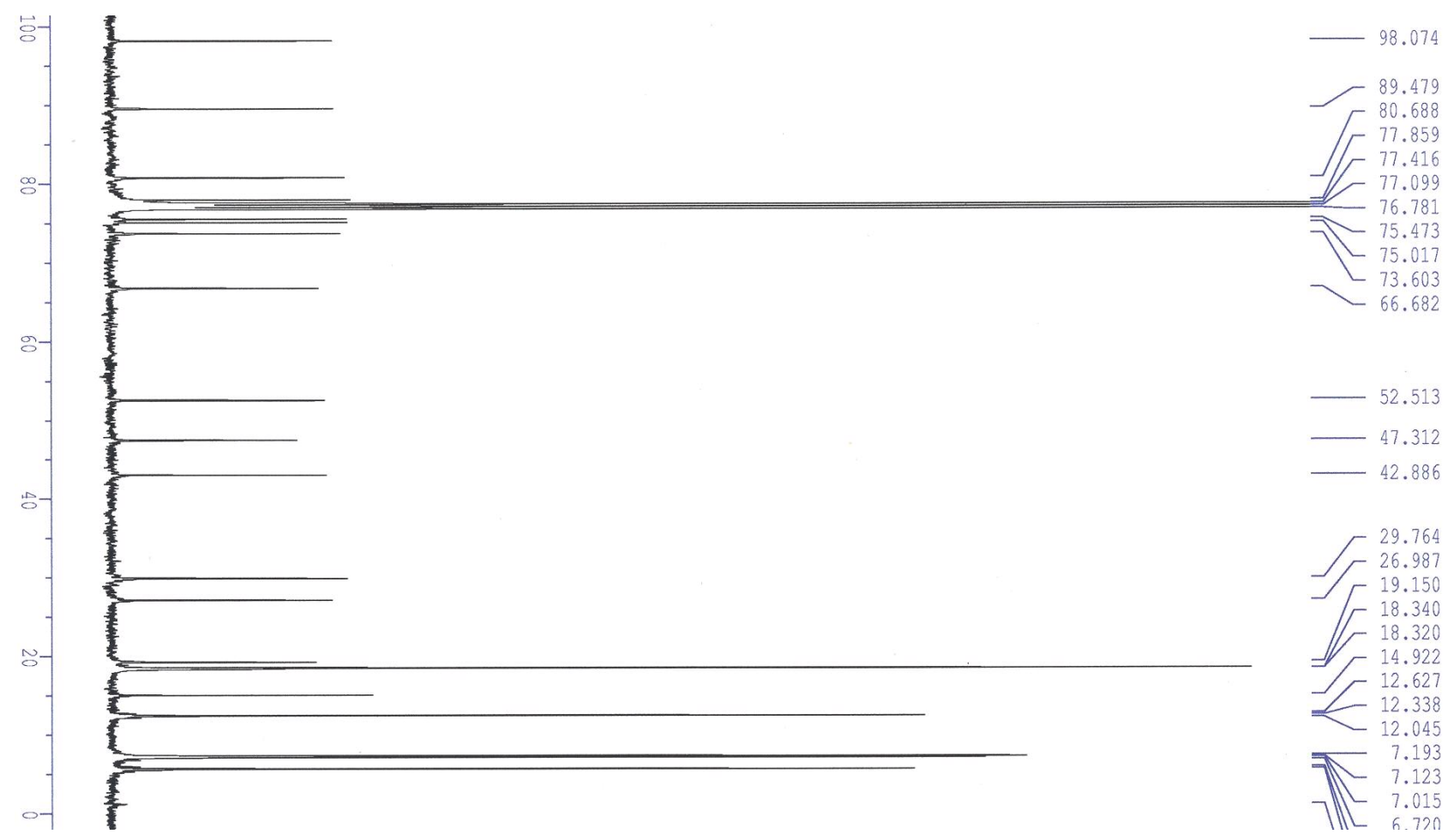


J. S. Clark et al. - Supporting Information
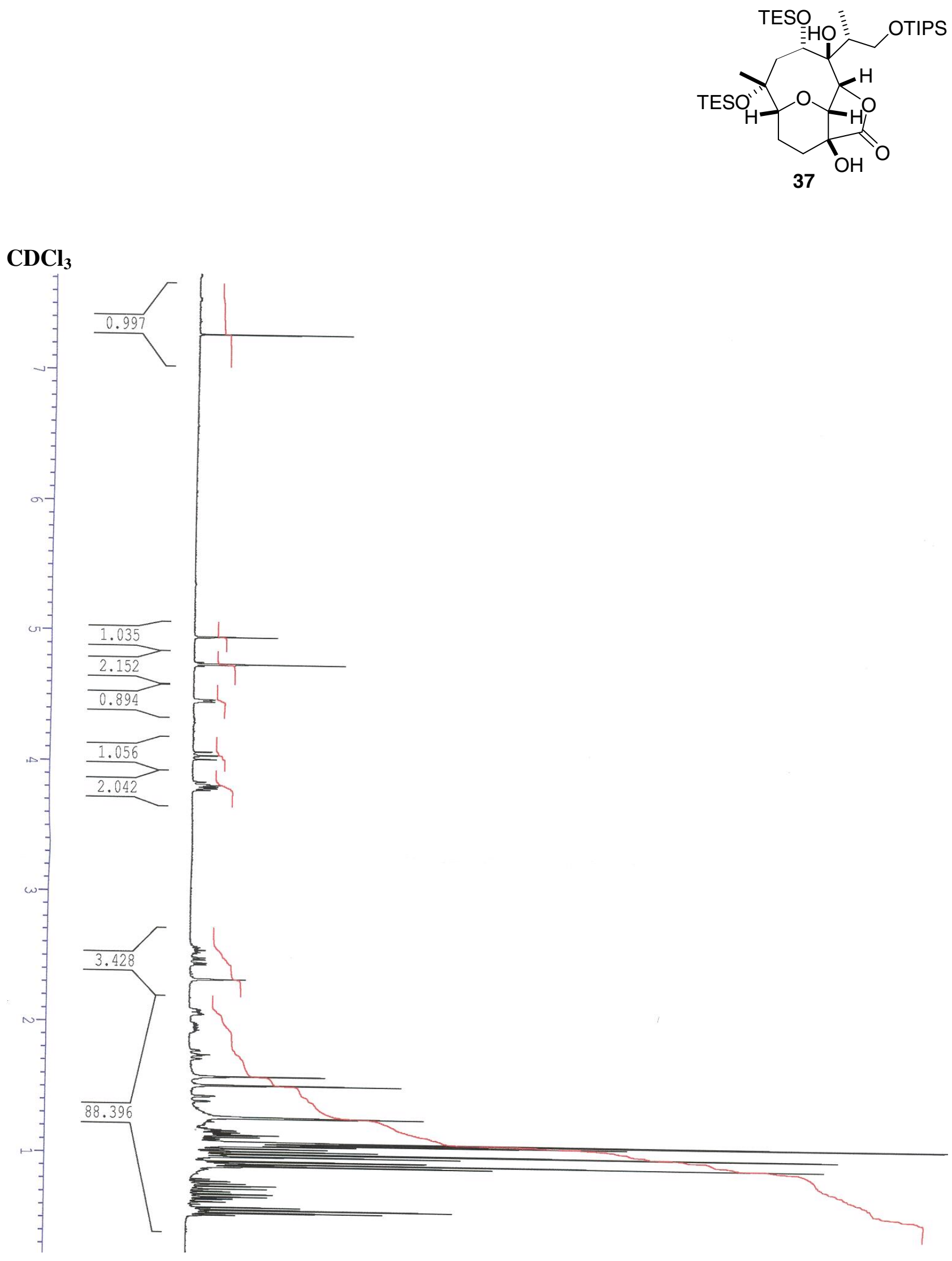

S49 
J. S. Clark et al. - Supporting Information
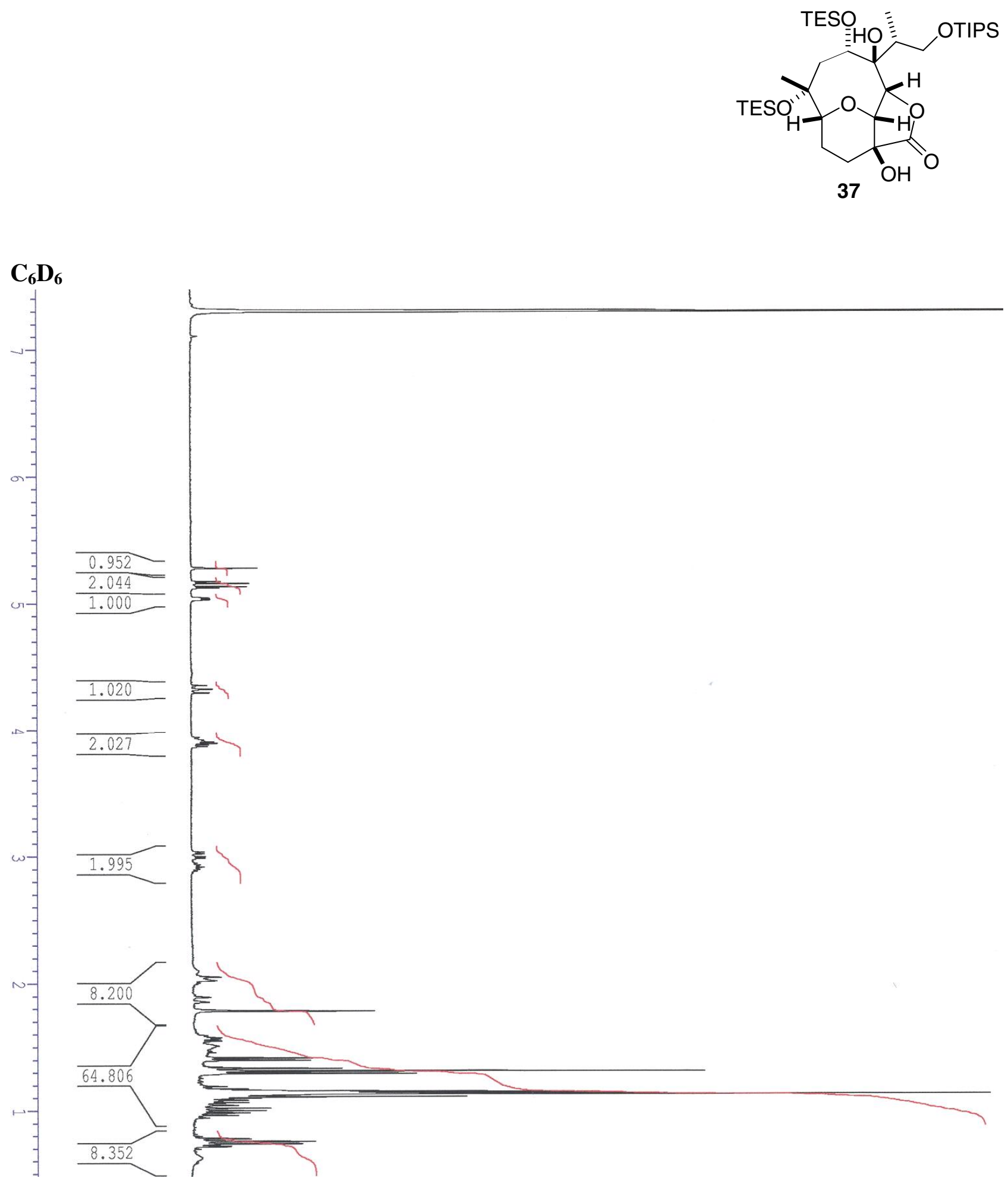
J. S. Clark et al. - Supporting Information
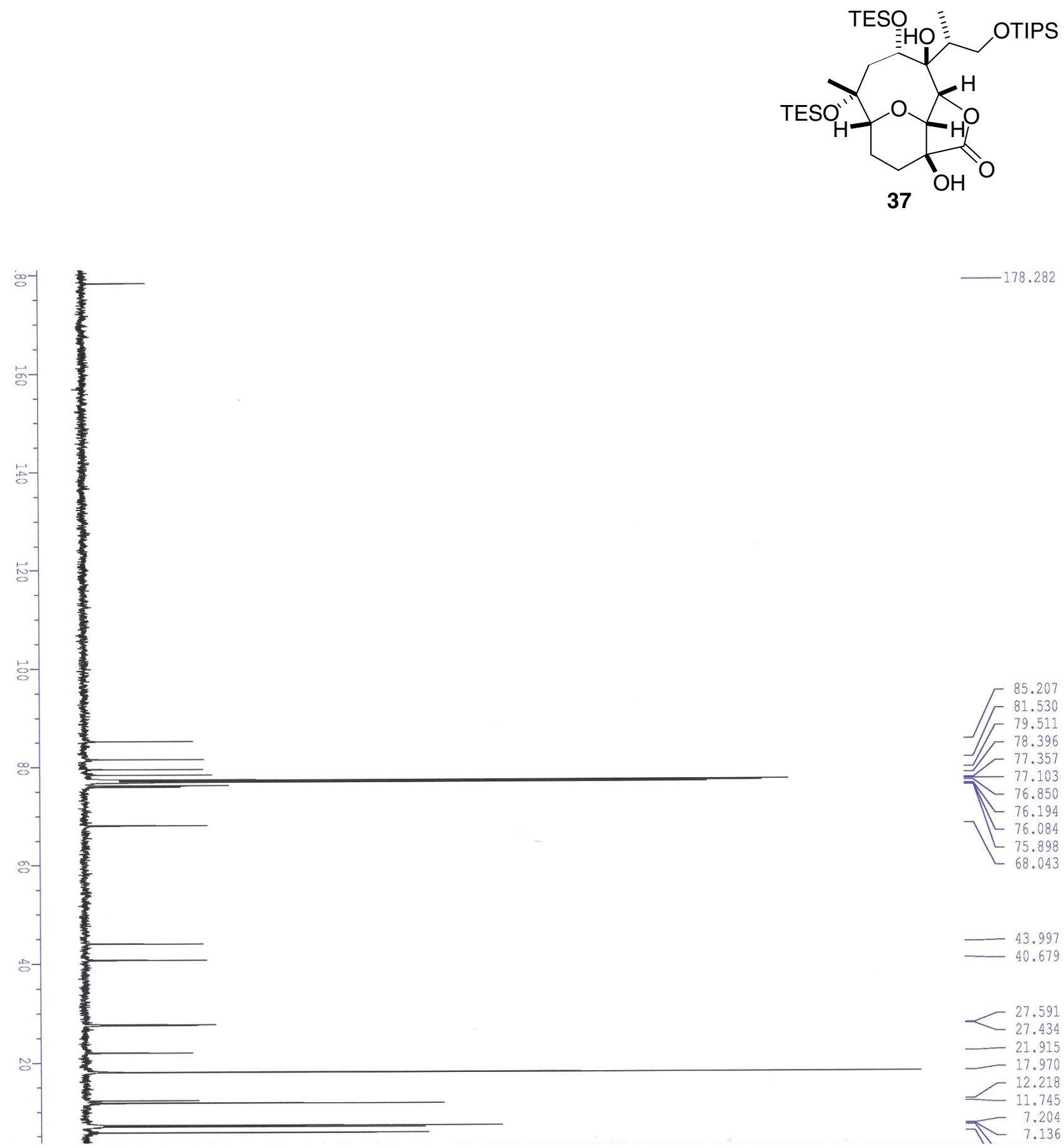
J. S. Clark et al. - Supporting Information

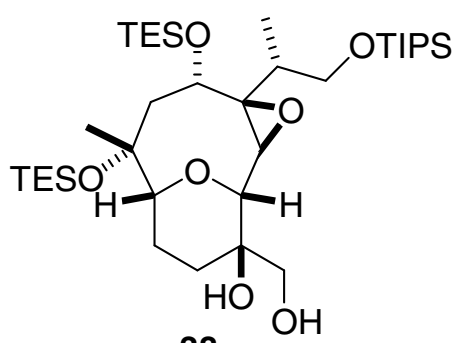

38

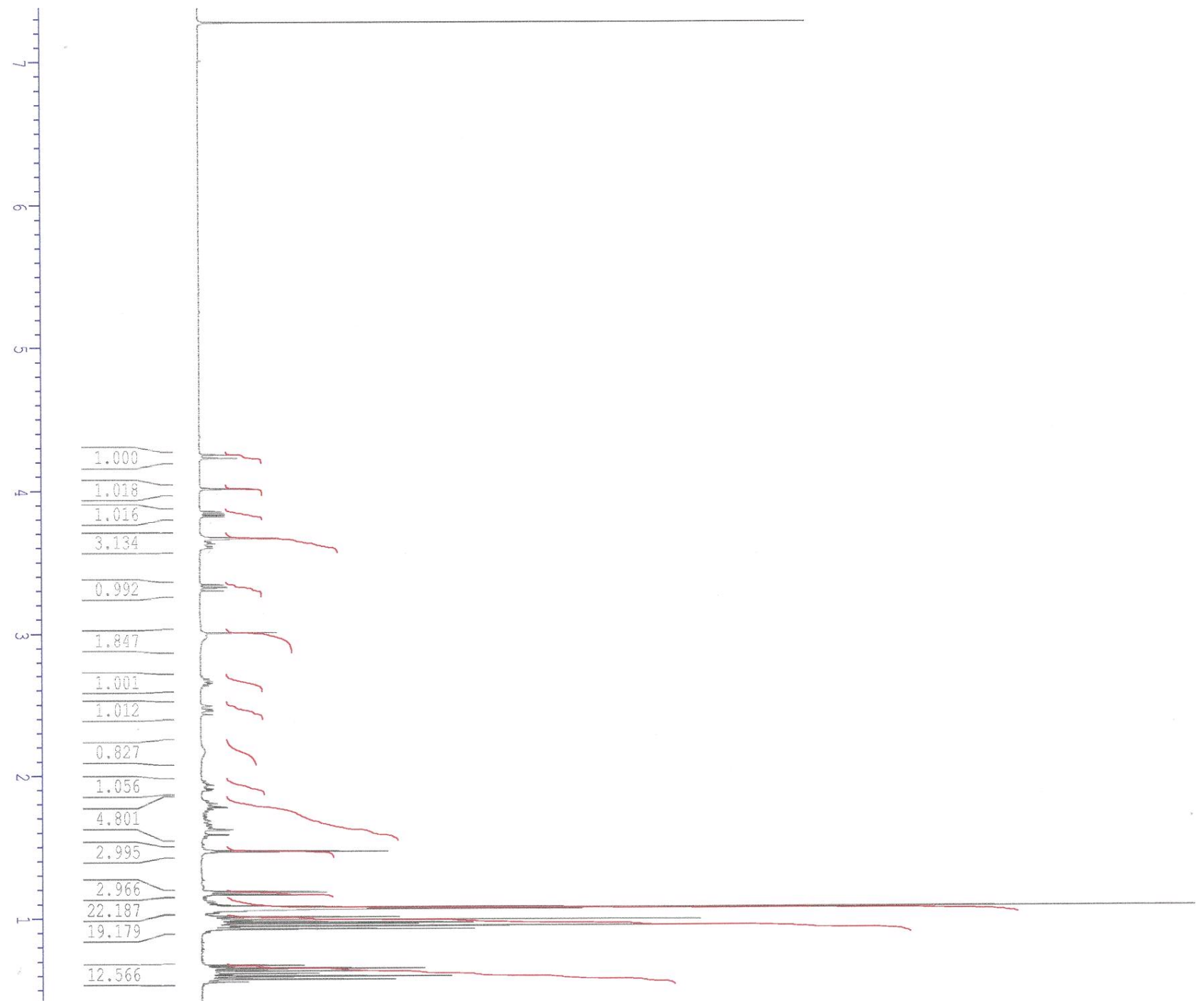


J. S. Clark et al. - Supporting Information

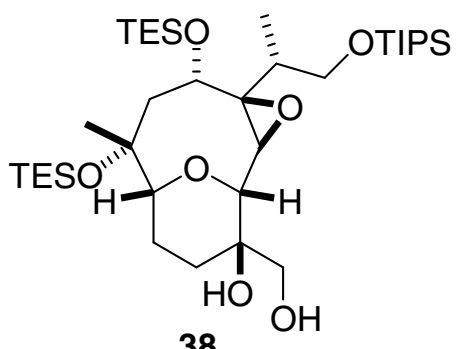

38
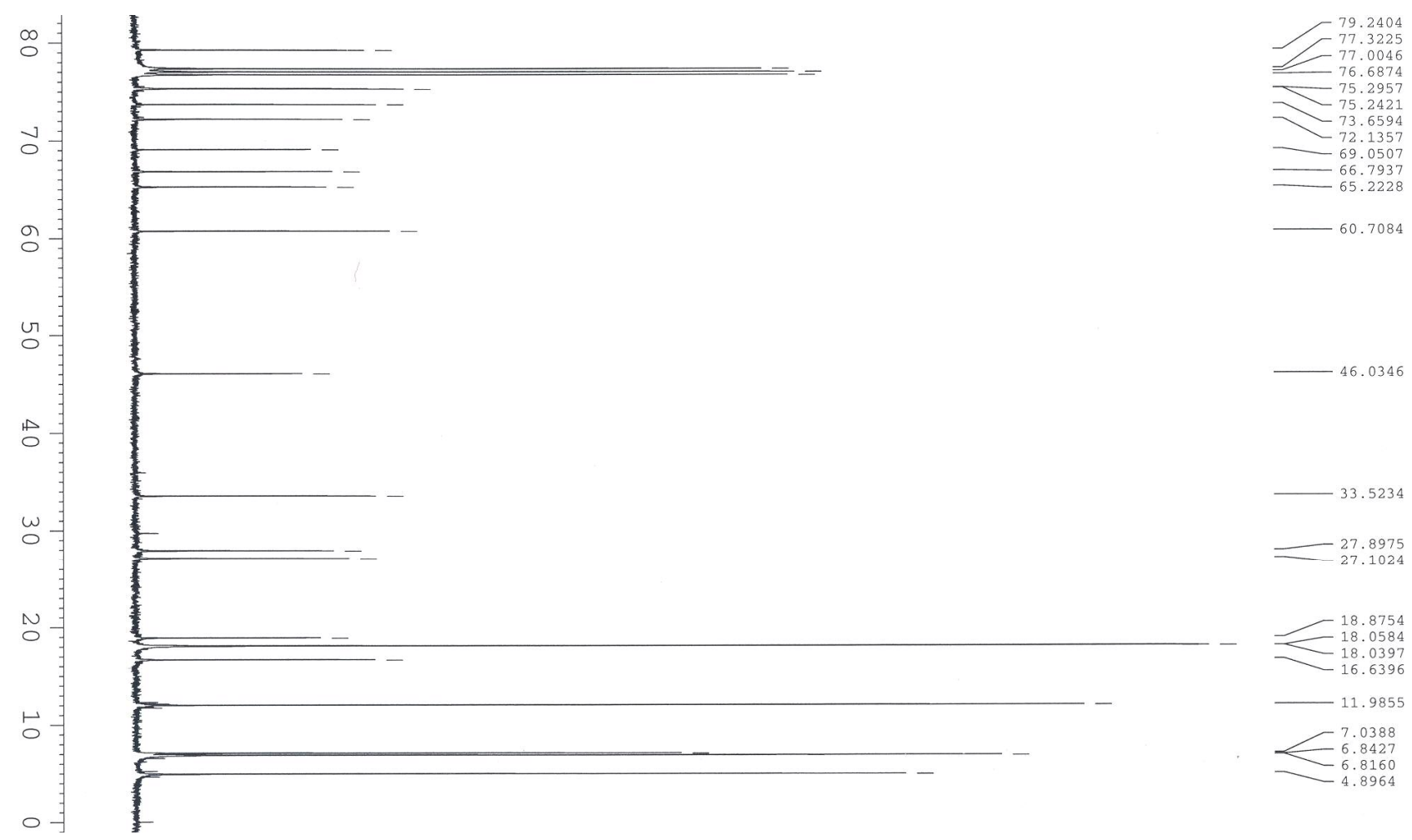


\section{J. S. Clark et al. - Supporting Information}
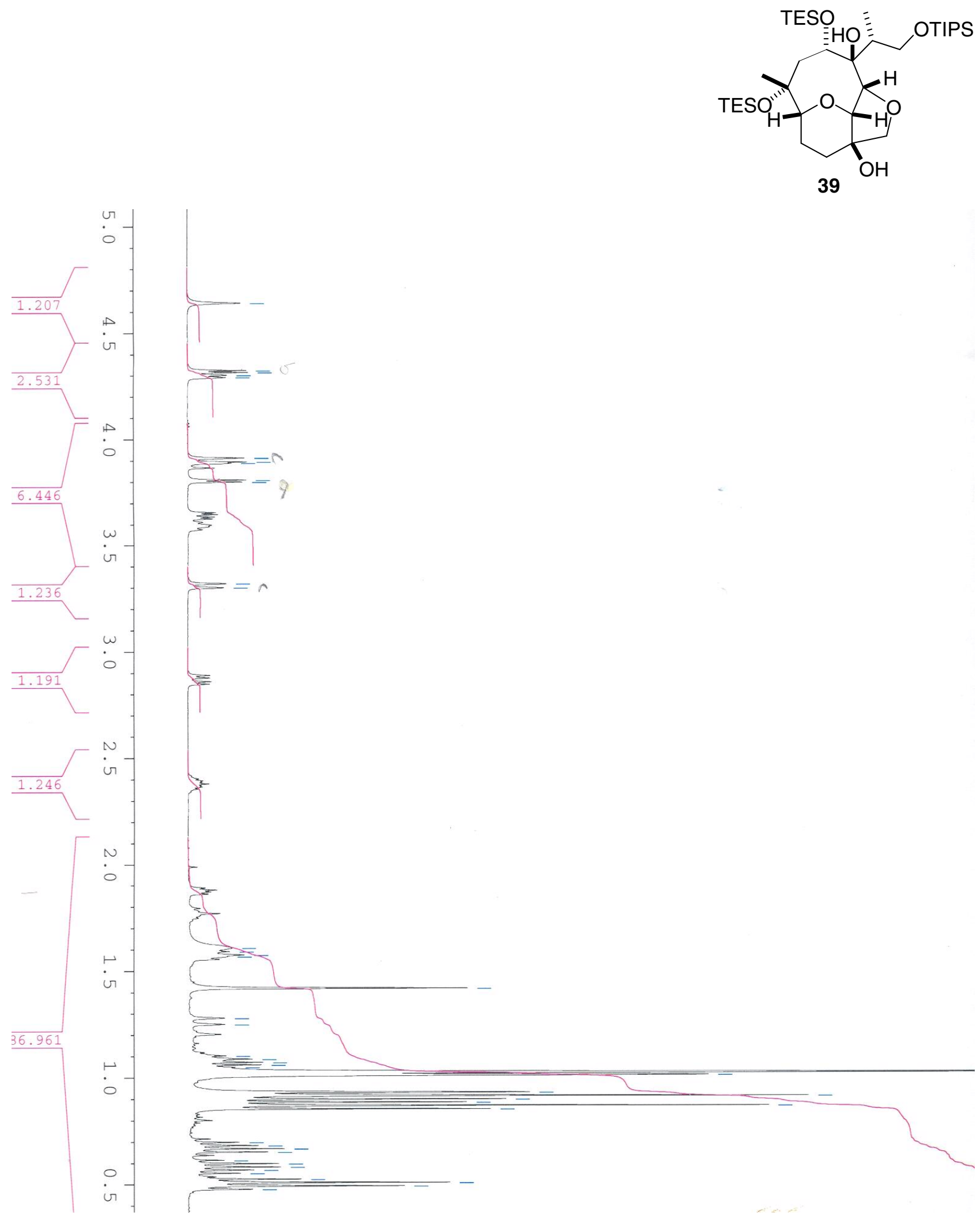
J. S. Clark et al. - Supporting Information
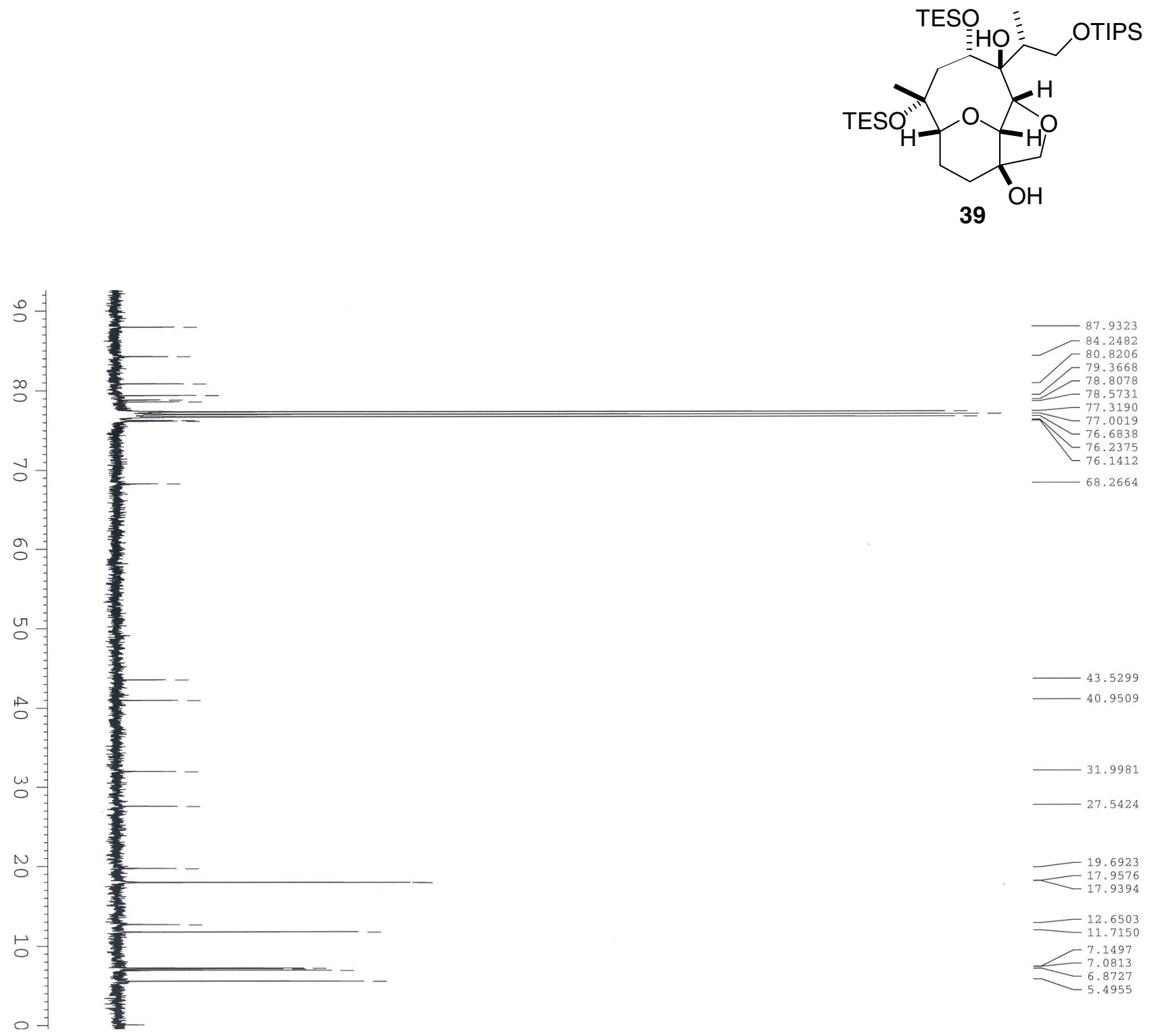

19.6923
$\mathbf{r}_{17.957} 17.9994$
17.96

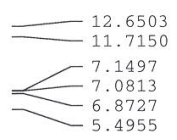


J. S. Clark et al. - Supporting Information
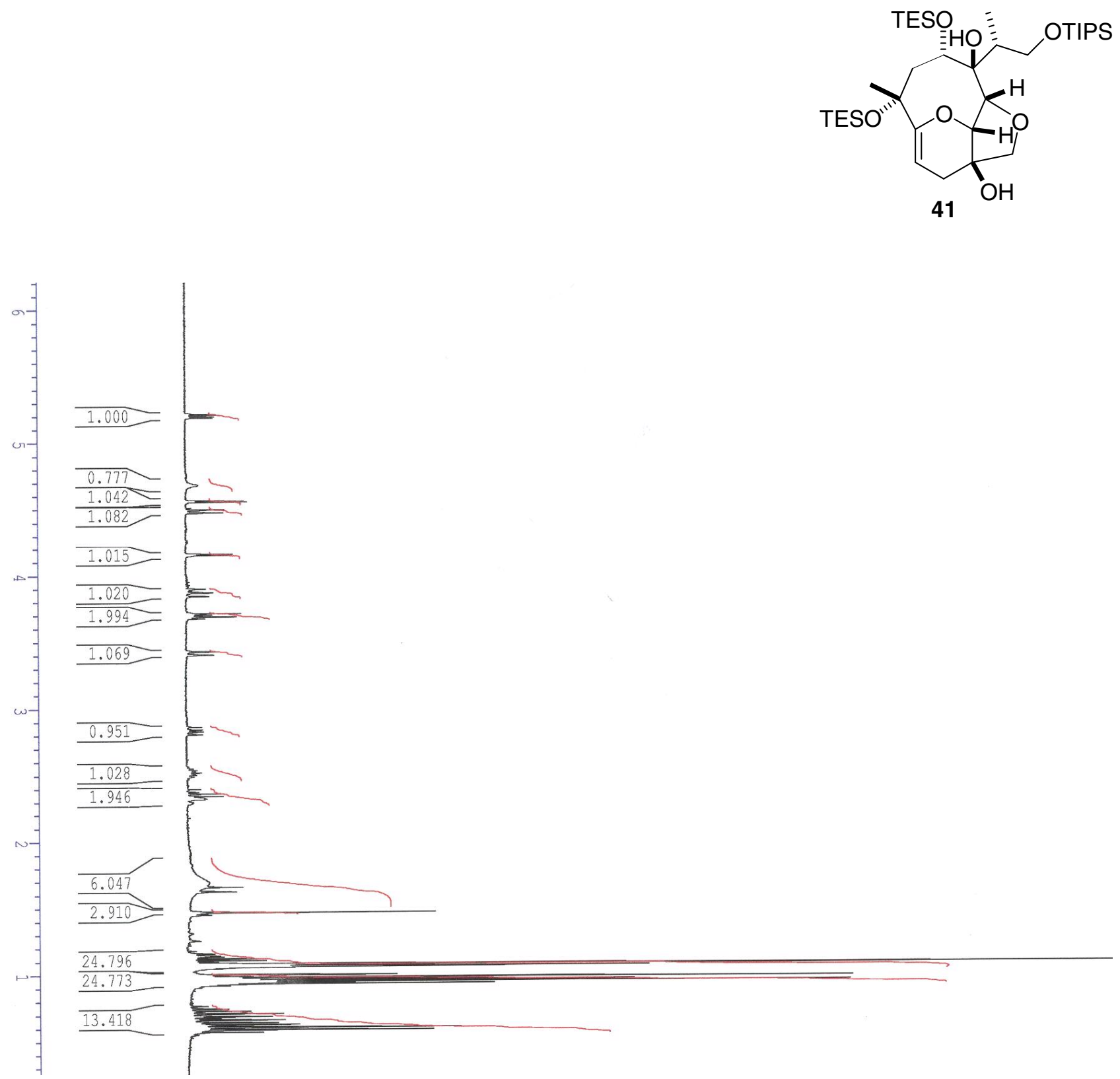
J. S. Clark et al. - Supporting Information
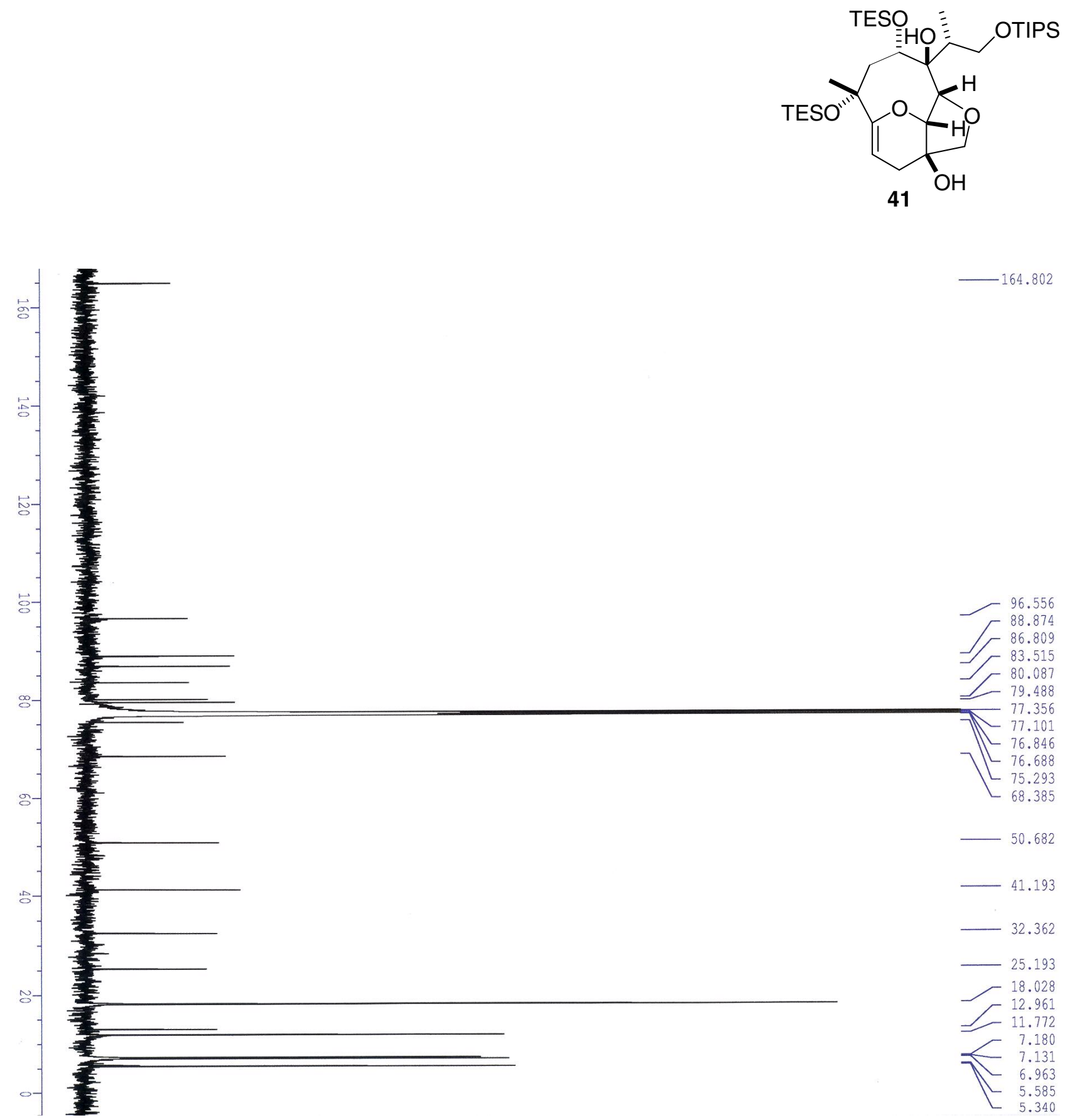

$-164.802$

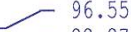

86.809

83.515

80.087
-79.488

77.101

$7 \begin{array}{r}-76.846 \\ -76.688\end{array}$

L 75.293

$-50.682$

$-41.193$

$-32.362$

$-25.193$

$-18.028$

F 12.961

< 7.180

$7-\quad .1363$ 
J. S. Clark et al. - Supporting Information

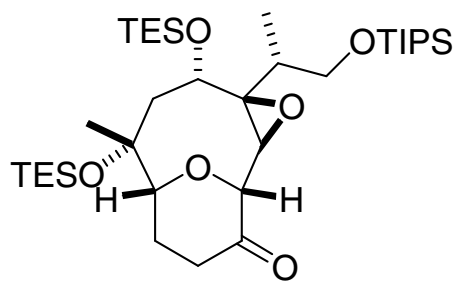

43

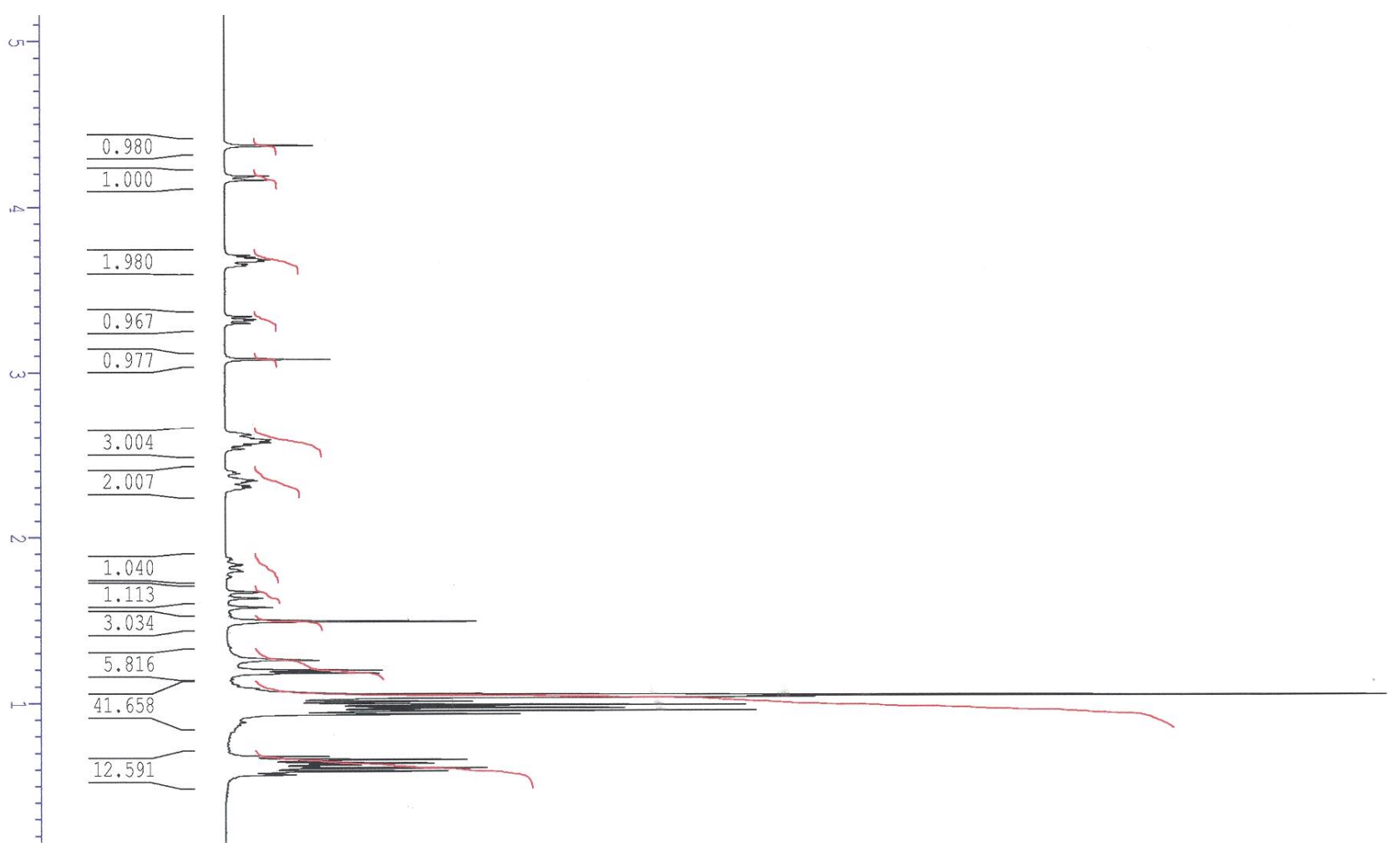




$$
E
$$


J. S. Clark et al. - Supporting Information
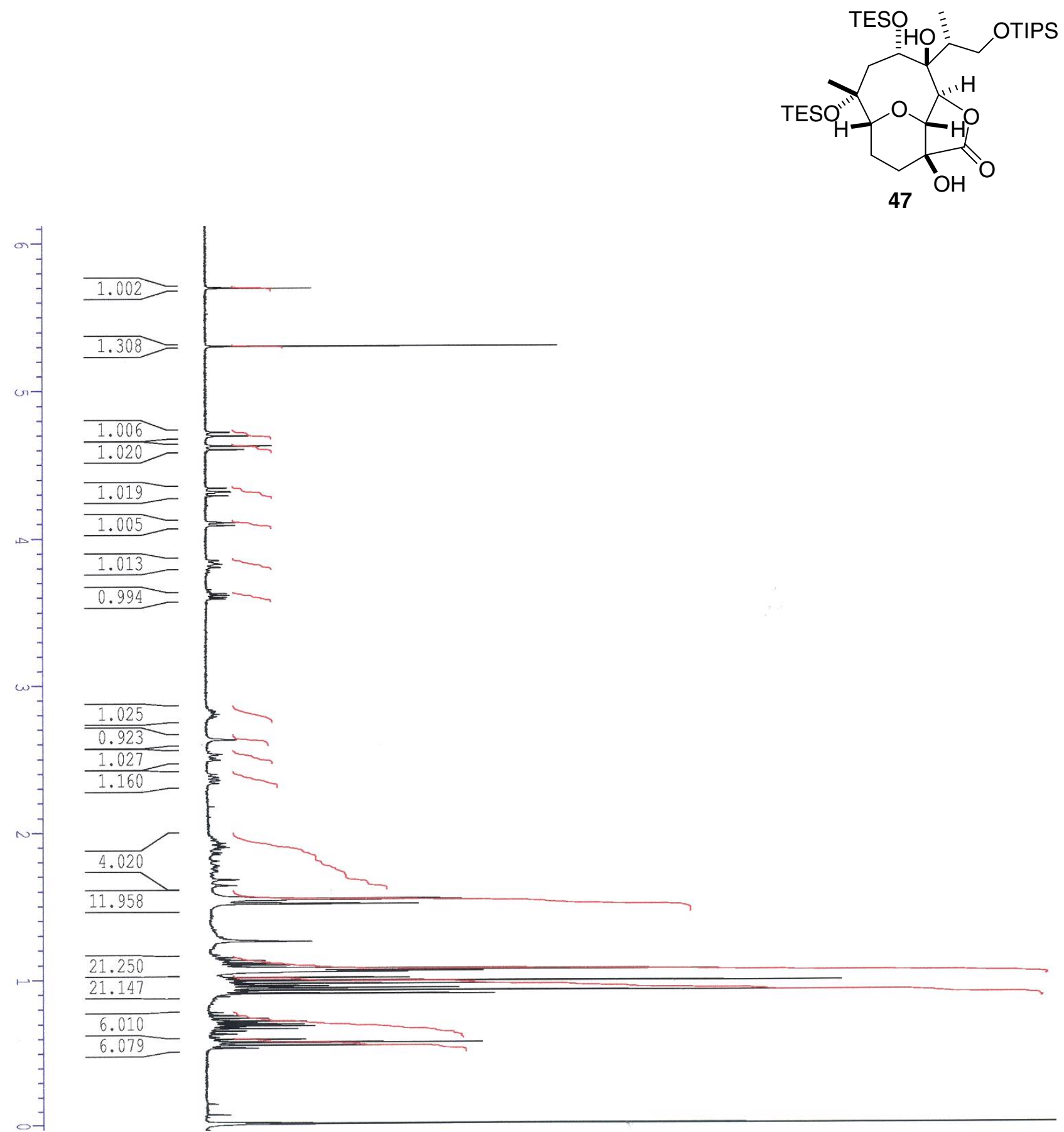
J. S. Clark et al. - Supporting Information
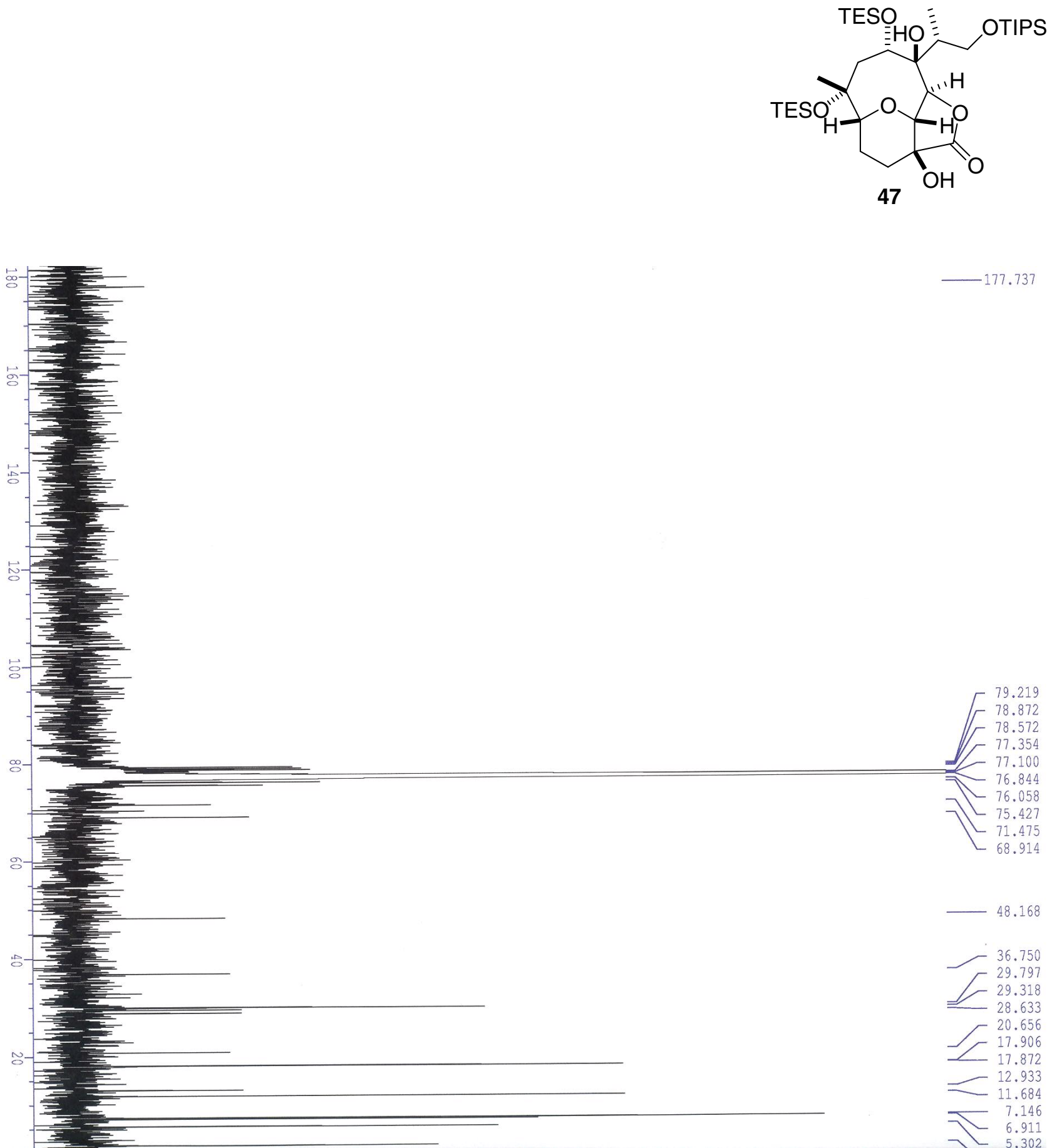

F 78.872

F 78.572

$F^{77.354}$

$-76.844$

- 75.427

L 68.914

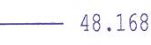

$-36.750$

F 29.318

$-28.633$

- 20.656

$\sim 17.906$

$-12.933$

11.684
7.146

- 6.911 